\title{
DESIGN AND DEVELOPMENT OF TWO TEST FIXTURES TO TEST THE LONGITUDINAL AND TRANSVERSE TENSILE PROPERTIES OF SMALL DIAMETER TUBULAR POLYMERS
}

\author{
A Thesis \\ presented to \\ the Faculty of California Polytechnic State University, \\ San Luis Obispo \\ In Partial Fulfillment \\ of the Requirements for the Degree \\ Master of Science in Biomedical Engineering \\ by
}

Carolyn Rae Berry

April 2011 
(C) 2011

Carolyn R. Berry

ALL RIGHTS RESERVED 


\section{COMMITTEE MEMBERSHIP}

TITLE:

AUTHOR:

DATE SUBMITTED:

COMMITTEE CHAIR:

COMMITTEE MEMBER: Lily Laiho, PhD

COMMITTEE MEMBER: Lanny Griffin, PhD

Carolyn Rae Berry

April 2011

DESIGN AND DEVELOPMENT OF TWO TEST

FIXTURES TO TEST THE LONGITUDINAL AND

TRANSVERSE TENSILE PROPERTIES OF SMALL

DIAMETER TUBULAR POLYMERS

Kristen O’Halloran Cardinal, PhD 


\begin{abstract}
DESIGN AND DEVELOPMENT OF TWO TEST FIXTURES TO TEST THE

LONGITUDINAL AND TRANSVERSE TENSILE PROPERTIES OF SMALL DIAMETER TUBULAR POLYMERS
\end{abstract}

Carolyn Rae Berry

Hundreds of thousands of vascular bypass grafts are implanted in the United States every year, but there has yet to be an ideal graft material to substitute for one's own autologous vessel. Many synthetic materials have been shown to be successful vessel replacements; however, none have been proven to exhibit the same mechanical properties as native vessels, one of the most important criteria in selecting a vascular graft material. Part of this issue is due to the fact that, currently, there is no "gold standard" for testing the longitudinal and transverse tensile properties of small diameter tubular materials. While there are ASTM and ISO standards that suggest ways to test tubes in their original form, many researchers have published tensile strength data based on cutting the tube and testing it as a flat sample. Thus, it was the aim of this thesis to understand, establish, and implement accurate tensile testing methods of small diameter polymers in their original, tubular state on Cal Poly's campus.

Two test fixtures were created based on specified design criteria in order to test materials in their tubular form in both the longitudinal and transverse directions. Both fixtures were successful in testing PLGA and ePTFE samples, and statistical data was gathered for the transverse test fixture. The new transverse test fixture was tested against the current method of testing, and a significant $(\alpha=0.05)$ difference between methods 
was established for ultimate tensile strength. This analysis, however, cannot determine which test method is more accurate, thus more extensive testing is required to verify the design of both fixtures. By developing a method for testing small diameter polymers in tubular form on Cal Poly's campus, it allows for more testing of various small diameter tubes and more comparative data to validate each design. It also demonstrates a need for a more detailed and widespread standardization of testing for small diameter tubes, especially in vascular substitute applications where the ideal vessel replacement has yet to be found. 


\section{Acknowledgements}

First and foremost, I would like to thank my thesis advisor, Dr. Kristen

O’Halloran Cardinal. Your passion for BMED, strive for perfection, and genuine

kindness have been an inspiration to me and the rest of the BMED students. I can't thank you enough for the countless hours you have put into my education.

A big thank you goes to John Anderson, my undergraduate design partner, without whom this project would not be complete. Thank you, John, for all of your hard work! I would also like to thank the members of my thesis committee, Dr. Lily Laiho and Dr. Lanny Griffin. I appreciate your support and help through this process.

To my parents, Mom and Dad, I have been unbelievably blessed with your love and positive encouragement every step of the way. I am so grateful to have followed in the footsteps of two bright engineers, but it is your compassion towards others and notion to do the right thing that have become invaluable to me over the years. I couldn't have done this without you.

Special thanks goes out to my siblings, Diana, Mark, and Noelle, for always cheering me on; and also to my Grandma, for your unconditional love. I would also like to thank my BMED crew, you know who you are, for getting me through so many late night hours in the lab, rigorous design projects, and not-so-dead dead weeks. Thanks to all of my friends for supporting me through this long endeavor; and to Chris, you continue to motivate me to complete my goals and to strive to be a better person. Thank you. 


\section{Table of Contents}

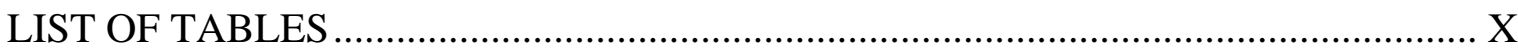

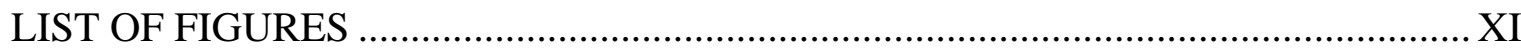

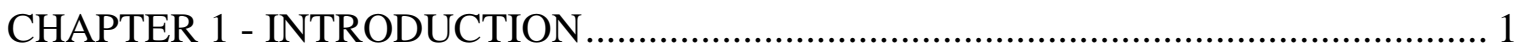

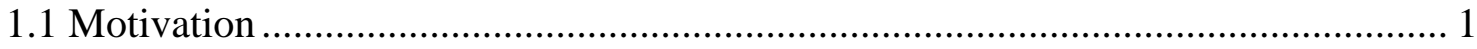

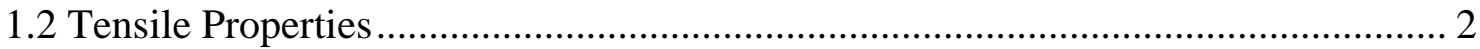

1.3 Small Diameter Tubular Polymers ........................................................................ 6

1.3.1 Fabrication methods .................................................................................... 7

1.3.2 Basic Material Characteristics ................................................................... 8

1.4 Tubular Biomaterial Applications .......................................................................... 9

1.4.1 Overview of Tubular Applications ..................................................................... 9

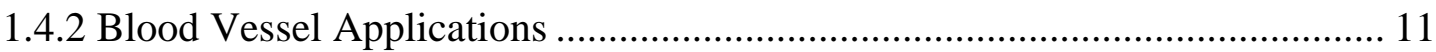

1.4.2.1 Native Blood Vessel Properties ................................................................... 12

1.4.2.2 Synthetic Vascular Graft Materials .............................................................. 13

1.4.2.3 Bioresorbable Vascular Graft Materials ................................................... 16

1.5 Testing the tensile properties of tubular constructs................................................. 18

1.5.1 Longitudinal tensile testing of tubular constructs............................................. 20

1.5.2 Transverse tensile testing of tubular constructs................................................ 26

1.6 Summary and Aims of the Thesis ......................................................................... 36

CHAPTER 2-TENSILE TESTING CAPABILITIES ON CAL POLY'S CAMPUS ... 38

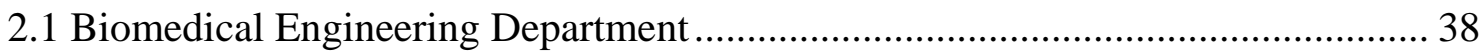

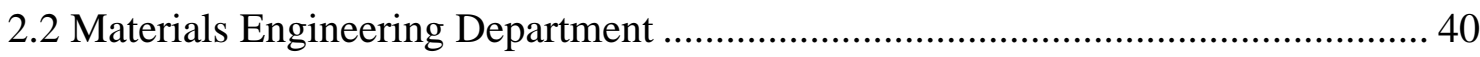

2.3 Mechanical Engineering Department................................................................... 43

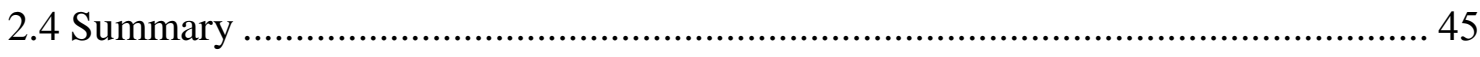

CHAPTER 3 - INITIAL DESIGN OF TRANSVERSE TEST FIXTURE....................... 46

3.1 Initial Design Models and Concepts .................................................................... 50

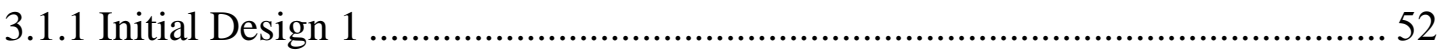

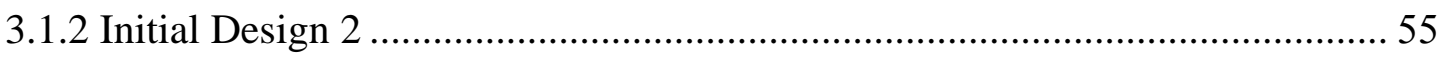

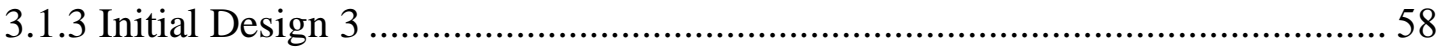

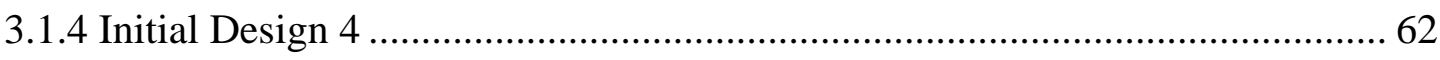


CHAPTER 4 - FINAL DESIGN OF TRANSVERSE TEST FIXTURE...................... 65

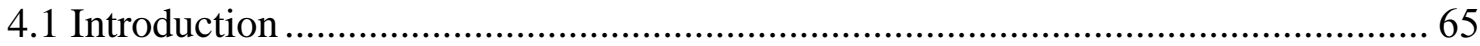

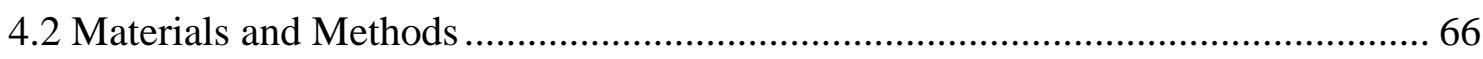

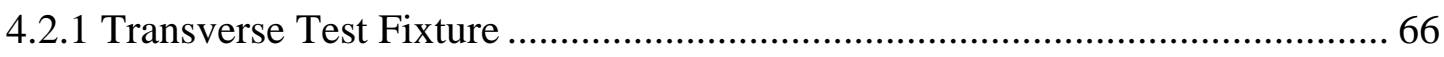

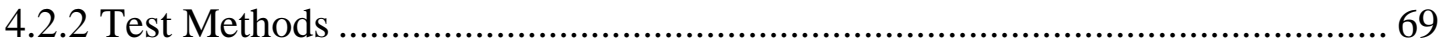

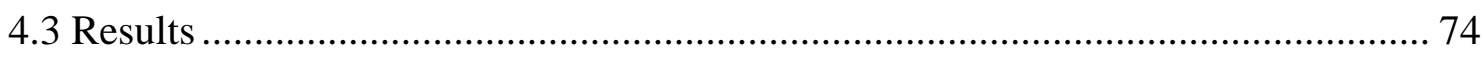

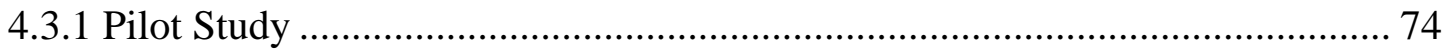

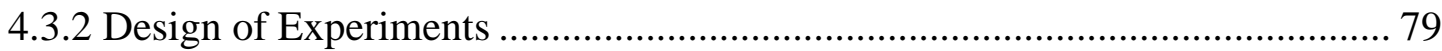

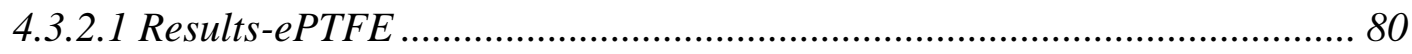

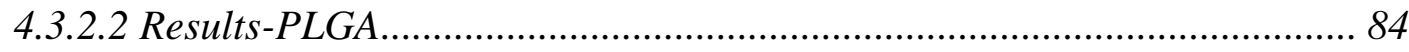

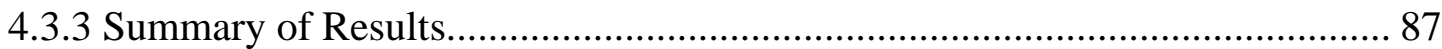

CHAPTER 5 - DESIGN OF LONGITUDINAL TEST FIXTURE ................................. 88

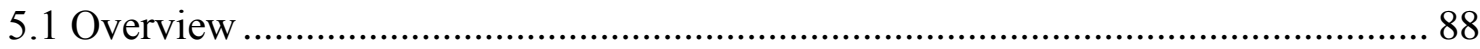

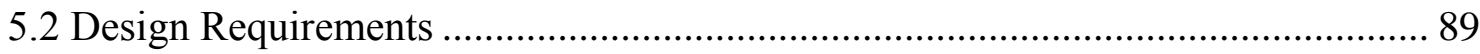

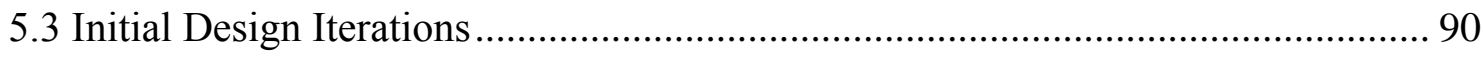

5.3.1 Initial Design 1 ........................................................................................... 90

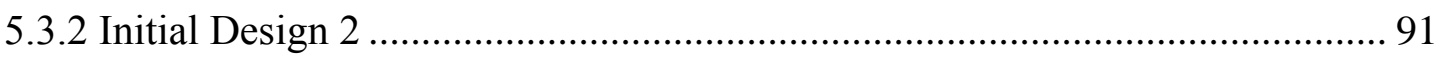

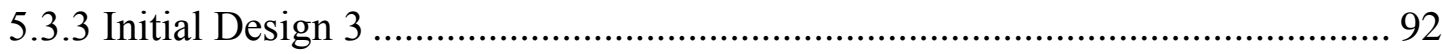

5.4 Final Design of Longitudinal Test Fixture .................................................................. 93

5.4.1 Testing the Final Longitudinal Test Fixture …………........................................ 96

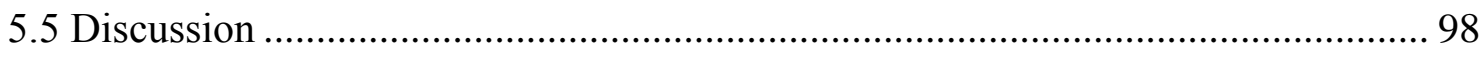

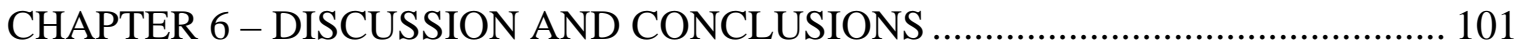

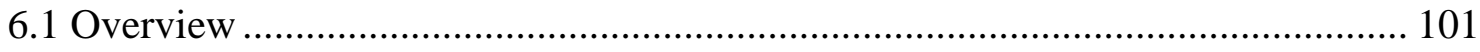

6.2 Final Transverse Test Fixture Design Challenges and Limitations ....................... 102

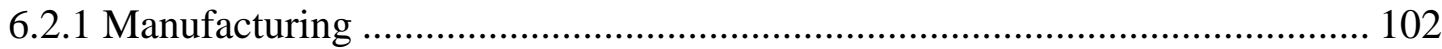

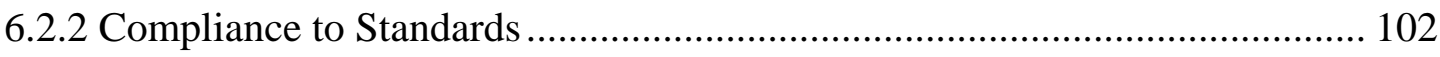

6.3 Discussion of Statistical Analysis for Transverse Test Fixture................................ 103

6.3.1 Stress-Strain Data for PLGA and ePTFE Testing ........................................... 104

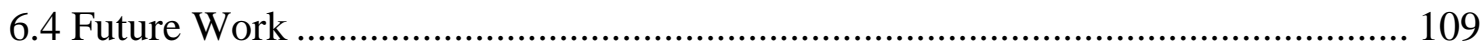

6.4.1 Randomization and More Extensive Testing................................................... 110

6.4.2 More Detailed Observations During Testing................................................... 111 
6.4.3 Burst Pressure Testing...................................................................................... 112

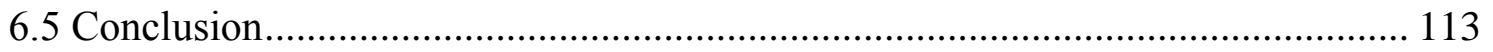

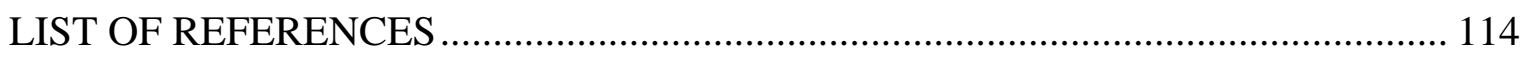

APPENDIX A: ASTM D 2290-STANDARD TEST METHOD FOR APPARENT HOOP TENSILE STRENGTH OF PLASTIC OR REINFORCED PLASTIC PIPE

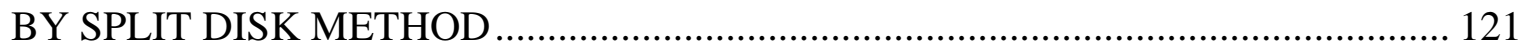

APPENDIX B: ISO 7198-DETERMINATION OF CIRCUMFERENTIAL TENSILE

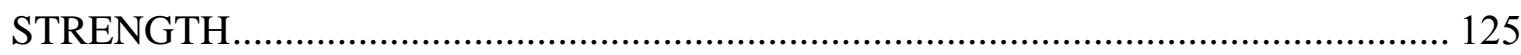

APPENDIX C: FINAL TRANSVERSE TEST FIXTURE ENGINEERED DRAWINGS 128

APPENDIX D: PROTOCOL FOR TESTING TUBULAR POLYMERS USING TRANSVERSE TEST FIXTURE ON INSTRON IN-SPEC MACHINE .

APPENDIX E: PROTOCOL FOR TESTING TUBULAR POLYMERS USING INSTRON IN-SPEC MACHINE (CURRENT METHOD OF TESTING) .................... 133 APPENDIX F: RESULTS OF EPTFE AND PLGA EXPERIMENTAL STUDIES COMPARING TRANSVERSE FIXTURE VS. CURRENT METHOD OF TESTING 138 APPENDIX G: FINAL LONGITUDINAL TEST FIXTURE ENGINEERED DRAWINGS 140 


\section{List of Tables}

Table 1: Summary Table of Documented Tensile Strengths of Native Blood Vessels Found in a Literature Review

Table 2: Summary Table of Documented Tensile Strengths of ePTFE Tubing Found in a Literature Review

Table 3: Summary Table of the Documented Tensile Strengths of Bioresorbable Graft Materials Found in a Literature Review.

Table 4: Summary Table of the Longitudinal Testing Methods of Tubular Constructs

Found in a Literature Review.

Table 5: Dimensions specified by ASTM D 2290 to prepare a material sample to be tested in tubular form using the split disk method [51].

Table 6: Summary Table of the Transverse Testing Methods of Vascular Graft

Constructs Found in a Literature Review

Table 7: Specifications for Instron In-Spec 2200 located in Building 192-330 on Cal Poly's campus

Table 8: Specifications for Instron 3369 located in Building 41-201 on Cal Poly's

Campus.

Table 9: Specifications for Instron 5584 located in Building 192-210 on Cal Poly's campus.

Table 10: Specifications for Instron 4400R located in Building 192-135 on Cal Poly's campus. 44

Table 11: ePTFE Pilot Study Results 75

Table 12: PLGA Pilot Study Results 77

Table 13: UTS results from testing six samples of PLGA in tubular form using the final longitudinal test fixture. 


\section{List of Figures}

Figure 1: Sample stress-strain curve typical of some polymers: (a) elastic deformation, (b) yield stress where linear-elastic deformation can no longer occur, (c) plastic deformation region, (d) point of fracture [7] ................................................................. 4

Figure 2: Diagram of the Longitudinal and Transverse Directions of a Tubular Material. The longitudinal direction runs along the axis of the length of the tube. The transverse direction is perpendicular to the axis of the tube.

Figure 3: SEM picture of ePTFE fibrils. Longer, thicker fibrils (or "nodes") are shown vertically in the photo, and are connected by smaller fibrils in between.

Figure 4: (a) Universal testing machine, (b) magnified view of grips holding dog bone shaped test sample [45]

Figure 5: ASTM D 638 outlines dimensions for different types of materials to be tensile tested in the longitudinal direction. Based on the category a specific material fits into, this table shows how the test sample should be prepared [46].

Figure 6: ASTM D 638 diagram shows metal plugs, shaded here with diagonal lines, to hold the shape of the plastic tube while testing for longitudinal tensile properties [46] . 22

Figure 7: Diagram of fixture specified to test the hoop tensile strength of plastic tubing according to ASTM Standard D 2290. D shaped blocks, shown to the right, are inserted into the tubular test sample then attached to the fixture with pins shown to the left [51]

Figure 8: This figure, taken from ASTM D 2290, shows the dimensions of a prepared tubular sample ready to tested using the split disk method described in the standard [51].

Figure 9: Split disk method of testing the tensile properties of a tubular specimen [53]. 30

Figure 10: Novel ring test method to determine transvers properties of tubular specimens. Yellow highlighted area shows center piece that reduces bending moment in sample. Red shapes illustrate end pieces within sample where the uniaxial force is applied [44]

Figure 11: Typical ring test method using to hooks or rods to pull apart ring, or tube, sample[44]

Figure 12: Ring test used to determine transverse tensile properties of tissue engineered blood vessels. Large black dots show the hooks pulling apart the specimen. Smaller black dots show the displacement of the sample over the course to the test [54].

Figure 13: A) A piece of PLGA prior to tensile testing, cut down the length of the tube. B) The sectioned piece of PLGA during a tensile test using the current method of testing at Cal Poly.

Figure 14: Instron In-Spec 2200 located in 192-330 ....................................................... 39

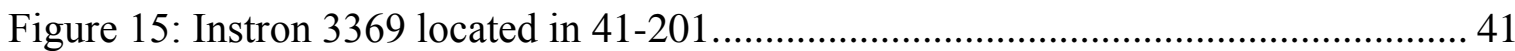


Figure 16: Instron 5584 located in 192-210.

Figure 17: Mechanical Engineering department tensile testing machines. On the left, Instron model 4400R. On the right, Instron model 1331. Both machines are located in Building 192-135.

Figure 18: Instron In-Spec grips fixate test fixture to apply test load to material. Round screw fasteners above grips ensure the fixture is tightly secured.

Figure 19: Initial sketch of transverse test fixture. The connection grips show where the fixture would be secured in the Instron machine's grips. The rod is what would support and apply the load to the tubular test sample. The shaded area on the rod shows where a tubular test sample would be placed.

Figure 20: The top semicircular shape shows a cross-section of the initial rod design and dimensions. The height is $1.4 \mathrm{~mm}$ in order to provide a $1 \mathrm{~mm}$ tolerance between opposing rods during testing. The bottom sketch shows half of the cross-sectional area of the rod.

Figure 21: SolidWorks model of initial design 1. Green arrow points to connection grip. Blue arrow shows rod and connecting pinhole to connection grip. Red arrow points to inset on rod to ensure test sample does not slide along rod.

Figure 22: Connection grips of initial design iteration 1. The two grips are shown as they would be aligned in the Instron testing machine.

Figure 23: Solidworks model of initial design 2. Two different rods were designed so they would be flush against each other, making sure the tubular test sample was kept in its original shape.

Figure 24: Rapid prototyped model of intial design 2 with sample ePTFE inserted in rods. The 90 degree angle circled in red shows where the tubular PLGA samples had

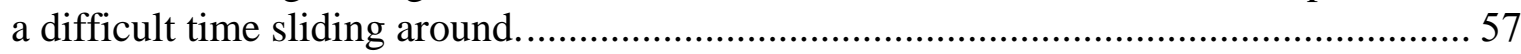

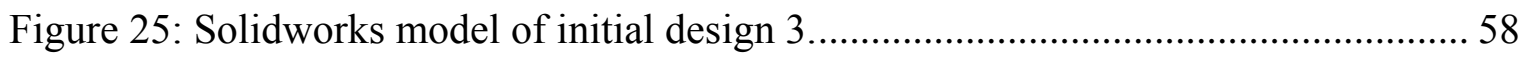

Figure 26: Rapid prototyped model of initial design 3. The top picture shows how bending both rods provided a flush surface against each other, while eliminating the difficult angle for stiffer materials to slide around. The bottom picture shows the slot, circled in red, that supports each rod.

Figure 27: Manufactured connection grip for initial design 3. Part was made out of 3/8" mild steel beam in Cal Poly's Mustang '60 machine shop.

Figure 28: Manufactured connection grip of initial design 3. Red circle shows where the slot is angled outward, making it unsuitable to support a rod.

Figure 29: Solid works model of design iteration 4, chosen for the final design. ............ 62

Figure 30: Rapid prototype model of final design with sampleof ePTFE attached.......... 63

Figure 31: Final design rapid prototype being tested in Instron grips to assure fit.

Sample of ePTFE attached to prototyped fixture.

Figure 32: CNC machined connection grip. 0.1485 " holes were drilled in order to press fit a 0.1495 " rod. 
Figure 33: Two rods were cut to $25 \mathrm{~mm}$ in length then ground down $0.075^{\prime \prime} 20 \mathrm{~mm}$ down the rod. Then each rod was press fit into the hole of each connection grip.

Figure 34: Final manufactured transverse test fixture.

Figure 35: The figure on the left shows a test sample prepared to be tested using the current method of cutting the tube then lying it flat to be tested. The red line shows the width of the test sample, and the figure on the right shows how the sample would be aligned in the Instron grips.

Figure 36: Drill press used to prepare notched samples of ePTFE and PLGA in tubular form.

Figure 37: A sample of ePTFE being tested in tubular form using the new transverse test fixture. Calipers were used to measure the gage length of each sample.

Figure 38: The top picture shows the prepared PLGA samples in tubular form. The bottom picture shows the samples after being tested. Note that for most samples tested in tubular form, one side of the tube fractured first.

Figure 39: ePTFE power curve for pilot study. Results show a sample size of 54 is needed to determine with a $95 \%$ level of confidence if there is a main effect on UTS due to test method.

Figure 40: ePTFE power curve for pilot study. Results show a sample size of 9 is needed to determine with a $95 \%$ level of confidence if there is a main effect on elastic modulus due to test method.

Figure 41: PLGA power curve for pilot study. Results show a sample size of 17 is needed to determine with a $95 \%$ level of confidence if there is a main effect on UTS due to test method.

Figure 42: PLGA power curve for pilot study. Results show a sample size of 17 is needed to determine with a $95 \%$ level of confidence if there is a main effect on elastic modulus due to test method.

Figure 43: One-way ANOVA residual plots for ePTFE elastic modulus. Plots show normally distributed populations and variances within three times of each other.

Figure 44: One-way ANOVA residual plots for ePTFE UTS. Plots show normally distributed population and variances within three times of each other.

Figure 45: ePTFE one-way ANOVA results for UTS. $\mathrm{P}=0.001<0.05=\alpha$, thus $\mathrm{H}_{\mathrm{o}}$ is rejected.

Figure 46: ePTFE one-way ANOVA results for elastic modulus. $\mathrm{P}=0.333>0.05=\alpha$, thus the test failed to reject $\mathrm{H}_{0}$.

Figure 47: One-way ANOVA residual plots for PLGA UTS. Plots show normal population distribution and variances within three times of each other.

Figure 48: One-way ANOVA residual plots for PLGA elastic modulus. Plots do not show normal population distribution and variances are unequal.

Figure 49: PLGA one-way ANOVA results for UTS. $\mathrm{P}=0.000<0.05=\alpha$, thus $\mathrm{H}_{\mathrm{o}}$ is rejected. 
Figure 50: PLGA one-way ANOVA results for elastic modulus. These results, however, are discounted due to unequal variances.

Figure 51: In order to test the tensile properties of tubular materials in the longitudinal direction, the samples are prepared by cutting along the length of the tube and laying the piece flat between the Instron grips as diagrammed above.

Figure 52: Rapid prototyped model of the first design iteration.................................... 90

Figure 53: Rapid prototyped model of the second design iteration............................... 91

Figure 54: Rapid prototyped model of the third design iteration

Figure 55: A) Solidworks model of the top portion of the longitudinal test fixture, with a semicircular notch cut out on the bottom and two holes placed on top for the screws to be secured. B) Solidworks model of the mandrel attached to the back plate, which slides inside the slot made on the top and bottom portion of the fixture.

Figure 56: A) Final manufactured longitudinal test fixture; B) L-wrench used to tighten screws onto fixture; C) Top portion of fixture; D) Screws used to secure top and bottom portions around mandrel; E) Mandrel connected to back plate.

Figure 57: Tubular sample of PLGA secured in test fixture and loaded onto Instron grips, ready to be tested.

Figure 58: PLGA stress-strain curve for samples tested in tubular form using the transverse test fixture.

Figure 59: PLGA stress-strain curve for samples tested using the current method of cutting the sample and laying it flat between the Instron grips.

Figure 60: ePTFE stress-strain curve for samples tested in tubular form using the new transverse test fixture.

Figure 61: ePTFE stress-strain curve for samples tested using the current method of cutting the sample and laying it flat between the Instron grips.

Figure 62: The ePTFE samples shown to the left were tested in tubular form while the samples on the right were tested after the sample was cut and laid flat between the Instron grips. Note the samples tested in tubular form fractured on only one side of the tube, but fractured in the middle of the notch. The samples that were cut fractured closer to where they were attached to the Instron grips. 


\section{Chapter 1 - Introduction}

\subsection{Motivation}

Synthetic arterial substitutes have been used in patients for over fifty years to treat coronary and peripheral artery disease [1]. Over the decades, many different materials have been meticulously studied in hopes of finding the optimum graft material to treat this disease [2]. With 600,000 coronary or peripheral vascular bypass graft surgeries being performed in the United States each year, there is yet to be an ideal answer to this conundrum [1].

The most preferable vascular replacement is the patient's own autologous graft; however, many patients do not have a suitable artery or vein for use [3]. When using a non-autologous material, the graft should ideally be non-thrombogenic and compatible at high blood flow rates. Additionally, the material must exhibit mechanical properties similar to native vessels. These characteristics become even more important when replacing smaller diameter vessels in low-flow rate environments [4].

In order to ensure the graft material has and maintains tensile properties similar to native blood vessels, appropriate material testing must occur [5]. There are currently no specific standards or methods that have been developed to test these properties in vascular graft materials. Thus, it is the aim of this thesis to understand, establish, and implement accurate tensile testing methods of small diameter tubular polymers on Cal Poly's campus in order to contribute to the standardization of this test procedure for vascular graft materials. 
In this chapter, the background and foundation of this thesis will be established. A brief introduction to tensile properties will be provided followed by a description of the two polymers of specific interest to this thesis. An overview of tubular biomaterial applications is then described along with common materials used in vascular graft applications. This chapter will also further explain the importance of knowing the tensile properties of vascular grafts and present different methods currently used for testing the tensile properties of small diameter tubular polymers. The last section in the introductory chapter will summarize the significance of this work and specify the goals and objectives of this thesis.

\subsection{Tensile Properties}

Tensile properties play an integral part in describing the mechanical properties of materials, and are applied in every engineering discipline [6-8]. Tensile properties are found from a tensile test and include the stress, strain, elastic modulus, and ultimate tensile strength of a material sample. Other properties that can be determined from a tensile test, but are not of relevance to this thesis, are yield strength, percent elongation at fracture, and percent reduction in area at fracture $[6,8]$. Stress $(\sigma)$ is determined by the average load or force (F) applied to the sample divided by the original cross-sectional area $\left(\mathrm{A}_{0}\right)$ of the sample. The units for stress are pounds force for U.S. customary and newtons per square meter $\left(\mathrm{N} / \mathrm{m}^{2}\right)$ or pascals $(\mathrm{Pa})$ for International System of Units (SI), where $1 \mathrm{~N} / \mathrm{m}^{2}=1 \mathrm{~Pa}$ [8]. The equation for stress is as follows:

$$
\sigma=\frac{F}{A_{0}}
$$


When a material is subjected to a uniaxial force, the sample extends in the direction of the force. The resulting displacement is defined as the strain $(\epsilon)$, or the ratio between the change in length $(\Delta \mathrm{L})$ of the sample in the direction of the force over the original length $\left(\mathrm{L}_{0}\right)$ of the sample. Units for strain are dimensionless as they are equal to inches per inch (in/in) for U.S. customary and meters per meter for SI [8]. The equation for strain is as follows:

$$
\begin{gathered}
\epsilon=\frac{\Delta \mathrm{L}}{L_{0}} \\
\Delta \mathrm{L}=\mathrm{L}-\mathrm{L}_{0} \\
\mathrm{~L}=\text { final length of the material sample }
\end{gathered}
$$

The results of a standard tensile test, described in detail later in this chapter, are typically presented in the form of a stress-strain curve, which is a plot of the material stress versus strain as the force is applied to the material sample [6]. Below is an example of a stress-strain curve typical to polymers; however, different materials produce a wide range of stress-strain relationships [7]. 


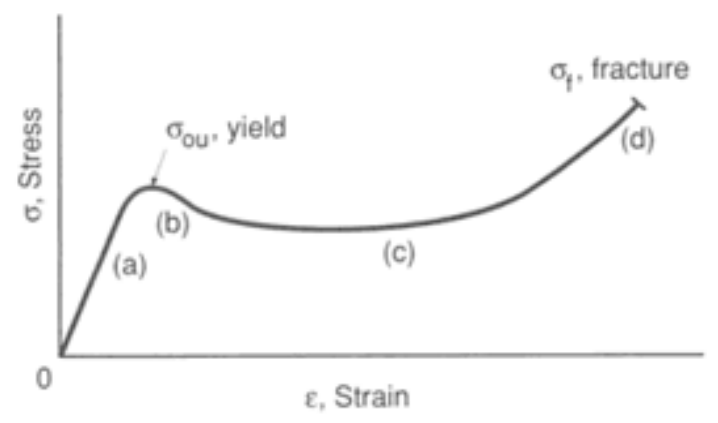

Figure 1: Sample stress-strain curve typical of some polymers: (a) elastic deformation, (b) yield stress where linear-elastic deformation can no longer occur, (c) plastic deformation region, (d) point of fracture [7].

The initial linear region (a) in the curve shows the elastic deformation of the material. A material is said to have experienced elastic deformation when it can return to its original dimensions after the force is removed. This is opposite of plastic deformation, in which the material becomes permanently deformed and cannot return to its original dimensions [8]. This occurs after the yield stress (b) has been reached. The stress-strain relationship, or slope, of the linear region is called the elastic modulus (E), also known as Young's modulus or the modulus of elasticity $[6,7]$. Accordingly, the elastic modulus is calculated using a form of Hooke’s law [8]:

$$
E=\frac{\sigma}{\epsilon}
$$

The units for the elastic modulus are pounds force per square inch (psi) for U.S. customary or pascals $(\mathrm{Pa})$ for SI [8]. 
A material's ultimate tensile strength $\left(\sigma_{u}\right)$ is defined as the maximum stress reached before fracture, shown as the highest point on the stress-strain curve [7, 8]. For some materials this point may be at the time of fracture such as with brittle materials; whereas with more ductile behavior, the load will reach a maximum point then begin to decrease before material fracture [7]. Therefore, to calculate the ultimate tensile strength, the highest load achieved at any point throughout the test $\left(\mathrm{F}_{\max }\right)$ must be divided by the original cross-sectional area $\left(A_{0}\right)$ [7]. The following equation is used for this calculation:

$$
\sigma_{u}=\frac{F_{\max }}{A_{0}}
$$

The aforementioned properties can differ within a tubular material sample based on the direction in which the uniaxial force is applied. Tubular tensile properties can be measured in two different directions. The longitudinal direction runs parallel to the length of the tube. The transverse direction, also called the radial or circumferential direction, runs perpendicular to the length of the tube. Figure 1 below diagrams both the longitudinal and transverse directions of a tubular material. The arrows indicate the direction in which the force would be applied to determine the longitudinal and transverse tensile properties.

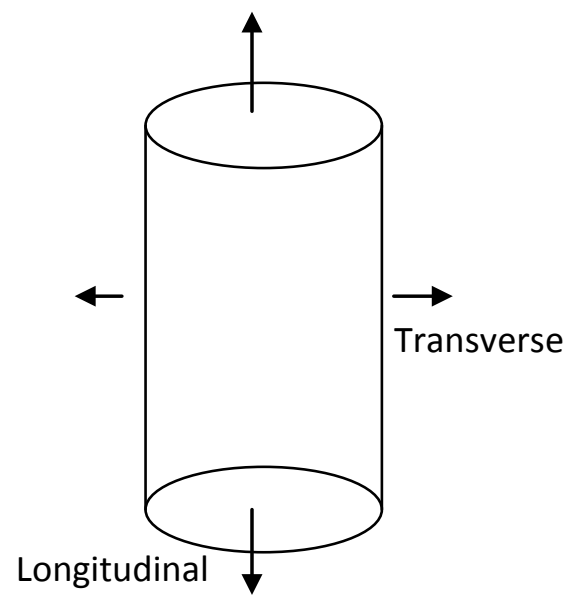

Figure 2: Diagram of the Longitudinal and Transverse Directions of a Tubular Material. The longitudinal direction runs along the axis of the length of the tube. The transverse direction is perpendicular to the axis of the tube. 
Tensile properties of tubular materials will be revisited later in this chapter; however, the reader must first be introduced to the types of tubular materials that are of interest to this thesis.

\subsection{Small Diameter Tubular Polymers}

For the purpose of this thesis, detail will be focused on two particular tubular polymers; however, an overview of similar materials will also be provided. Current research in the biomedical field involves testing and evaluation of many different small diameter polymer tubes [1, 9-11]. A vascular tissue engineering research group at Cal Poly, San Luis Obispo, currently under the helm of Dr. Kristen O'Halloran Cardinal, is interested in expanded poly(tetrafluoroethylene) (ePTFE) and poly (lactide-co-glycolide acid) (PLGA). These materials are specifically being researched for the use of in vitro "blood vessel mimics" (BVM) as a means to evaluate endothelialization of bare metal stents [12]. The purpose of this research is to develop testing methods for stents and other cardiovascular implants prior to in vivo testing by imitating a blood vessel environment in a bioreactor system. ePTFE is currently widely used as a vascular graft substitute and, pertaining to this research, serves as a scaffold in which cells can grow in BVM applications $[1,13,14]$. While ePTFE is advantageous in that it is biocompatible, able to be sterilized and available in desired size and dimensions, it is also expensive and not always ideal for desired applications in Cal Poly's BVM lab. Thus, the tissue engineering group developed an in-house fabrication method for electrospun scaffolds. The material chosen for this fabrication was PLGA, which exhibited properties appropriate for cell cultivation. PLGA is also inexpensive and able to be sterilized inhouse making it the most accessible and available material to use for Cal Poly's BVM lab 
[12]. The focus of this thesis will be on ePTFE and PLGA materials as they are the primary materials of interest for tensile testing tubular constructs on Cal Poly's campus.

\subsubsection{Fabrication methods}

ePTFE is fabricated using an extrusion process that begins with poly(tetrafluoroethylene) (PTFE) in a fine powder resin. The powder resin is formed into a paste using a specific lubricant, and then subsequently ram extruded into a solid tubular polymer. This tubular construct is then stretched at extremely high temperatures to form a microporous tube $[15,16]$.

PLGA, one of the most widely used materials in tissue engineering, is a copolymer of poly(DL-lactide) acid (PDLLA) and poly(glycolide) acid [17-19]. When fabricated using an electrospinning technique, the mole fractions between the two copolymers of PLGA typically range between 50:50, 75:25, and 85:15 (lactide:glycolide) [17-19]. Electrospinning uses an electric field to eject a charged polymer solution from a capillary tube onto a grounded metal collector. As the solution travels from the tube to the collector, the solvent evaporates, leaving behind the desired polymer as a non-woven fabric [20]. Processing parameters for electrospinning vary depending on the desired application. In Cal Poly's BVM lab, the polymer solution consists of $15 \mathrm{wt} \%$ PLGA dissolved in chloroform $\left(\mathrm{CHCl}_{3}\right)$. In order to achieve the tubular form of a blood vessel a mandrel is placed on the ground collector which rotates and translates back and forth to form the specified dimensions of the material [12]. 


\subsubsection{Basic Material Characteristics}

ePTFE has a node-fibril structure with an average intermodal distance of $30 \mathrm{um}$, where fine fibrils connect larger, solid nodes together [16]. The porous structure of ePTFE encourages cell adhesion in vascular graft applications, and its electronegative luminal surface is antithrombotic $[1,4,14]$. ePTFE is non-biodegradable and has shown success in lower limb bypass grafts (7-9 mm inner diameter); however, the patency, or openness of the vessel five years after implantation is shown to dramatically decrease [4].

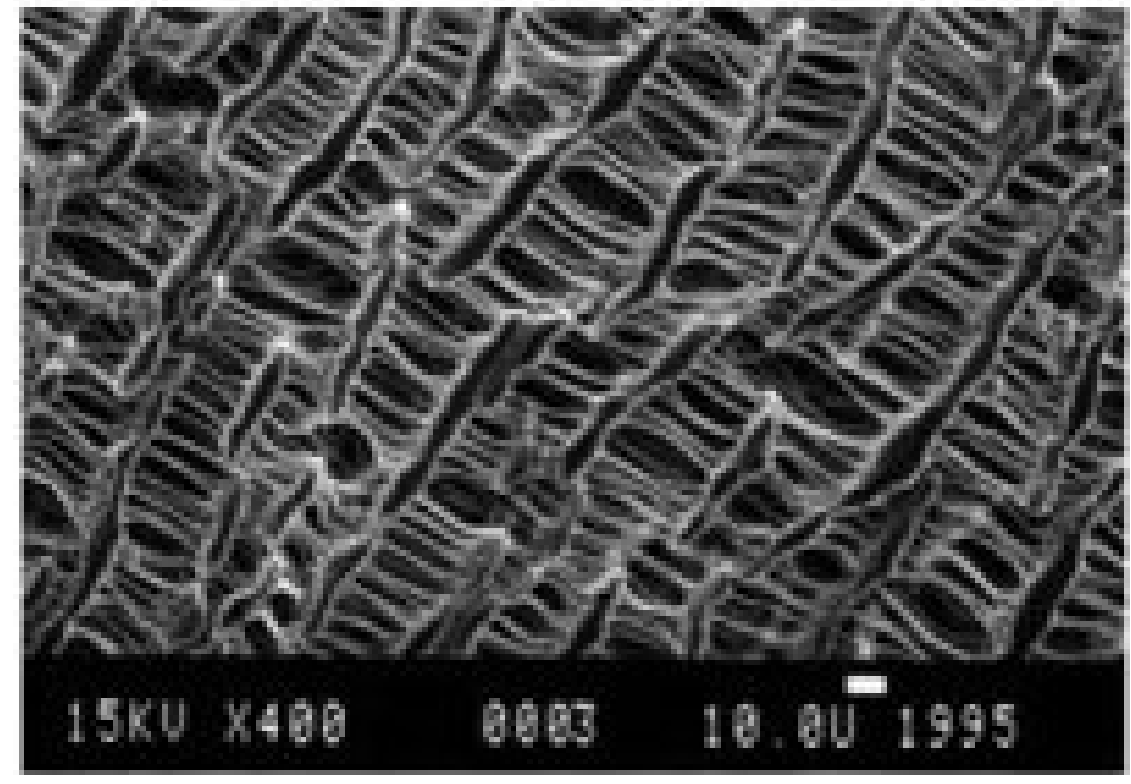

Figure 3: SEM picture of ePTFE fibrils. Longer, thicker fibrils (or “nodes”) are shown vertically in the photo, and are connected by smaller fibrils in between.

Unlike ePTFE, PLGA is a biodegradable polymer whose degradation timeframe can be projected as well as controlled based on fabrication processing parameters [17, 18]. While PLGA has been used in a wide variety of tissue engineering applications, the mechanical integrity of the tissue construct still decreases as the polymer degrades. Due 
to the lack of collagen and elastic fiber production in the construct, the mechanical properties are not recovered [14]. Thus, researchers have been incorporating collagen into their PLGA scaffolds to increase cell binding as well as smooth muscle and endothelial cell growth $[12,18]$. With smooth muscle cell proliferation, more collagen can be produced thereby retaining appropriate mechanical properties in the structure [1]. Specific mechanical and tensile properties for PLGA as well as ePTFE will be discussed in subsequent sections, while this next section will provide a basic overview of tubular biomaterial applications.

\subsection{Tubular Biomaterial Applications}

\subsubsection{Overview of Tubular Applications}

Before giving a detailed summary [21] of the tubular biomaterial application of interest to this thesis, a brief review of common tubular biomaterial applications will be provided. There are several small diameter tubular biomaterial applications in which there is interest in the material's mechanical properties [21-23]. These applications include catheters, neural scaffolds, stents, and vessels, each of which will be briefly discussed below.

Catheters are one example application where tubular mechanical properties are important [22, 24]. For hemodialysis catheters, catheter-related infections are fairly common, thus antimicrobial coatings are used as a means to prevent infection. In order to make sure these coatings do not compromise the catheter's mechanical integrity, tensile tests along with other mechanical tests are performed to ensure safety for the patient [25]. The tensile properties of catheters are also important to prevent the catheter 
from breaking upon insertion or removal from the patient [22, 24]. With specific regard to spinal-bore catheters used in continuous spinal anesthesia (CSA), manufacturers try to make the catheter as small as possible $(<1 \mathrm{~mm}$ ID) to avoid the chance of postdural puncture headache [24]. Other research has studied the mechanical properties of catheters punctured by surgical blades or needle bevels, specifically of epidural catheters [22]. While catheter breakage is unlikely and infrequent, knowing the mechanical properties of these tubes is essential to ensure the highest rate of safety and effectiveness $[22,26]$.

Nerve regeneration is also an area in which the mechanical properties of tubular materials must be evaluated. Tubular scaffolds, ranging between $1.5 \mathrm{~mm}$ and $4 \mathrm{~mm}$ inner diameter, are used as a substitute for auto grafts to repair damaged nerves [23, 27]. The scaffold provides protection from surrounding connective tissue and room for nerve axons to extend and grow [23]. Along with upholding an environment conducive to nerve growth, the tubular scaffold must endure the suturing of the implant to the existing tissue as well as the normal, daily movements of the patient during tissue growth [27]. Therefore, the mechanical properties of these tubular scaffolds must be adequate to withstand the aforementioned forces. Furthermore, the scaffolds must not lose any of these properties after standard sterilization techniques [27].

Similar to hemodialysis catheters, urinary stents and catheters can often result in urinary tract infections; thus, different antimicrobial coatings are frequently studied to resolve this issue $[21,28,29]$. An ideal urinary stent must have excellent tensile properties while also having the ability to elongate [21]. Additionally, any coatings to fight infection must not have any negative impact on the tensile properties [28]. When 
urinary catheters have been implanted for a long duration of time, they must be removed in a safe and effective manner. To prevent any fracture to the catheter, the urinary catheter material must withstand the force of removal [29]. While it is important to be aware of the variety of tubular biomaterial applications, one of the most studied applications in the biomedical industry involves blood vessel regeneration and repair [1, $2,13]$.

\subsubsection{Blood Vessel Applications}

There is currently a worldwide effort in science and engineering to develop new methods for treatment of blood vessel dysfunction $[3,9,14,19]$. This research is not being conducted without need. Atherosclerosis is the largest cause of mortality in the United States, including coronary and peripheral artery disease [30]. Approximately 100,000 patients each year who require vascular grafts do not have autologous veins or arteries suitable for transplant [3]. Synthetic polymeric vascular grafts have been successfully used for large diameter vessel applications ( $>6 \mathrm{~mm}$ inner diameter); however, for small diameter vessels, tissue engineered vascular grafts on biodegradable scaffolds seem to be the most promising treatment [10]. For a vascular graft to be safe and effective in a patient, it must have tensile properties similar to those in a native blood vessel along with other important characteristics [2]. A detailed explanation of this requirement will be provided later in this chapter; however, the following sections will describe documented tensile properties of native blood vessels as well as different materials used in vascular graft applications. 


\subsubsection{Native Blood Vessel Properties}

Currently, there is minimal literature published documenting tensile properties of native blood vessels. Kurane et al. studied the circumferential tensile properties of porcine carotid arteries, which were similar to tensile properties of human atherosclerotic carotid arteries studied by Teng et al and the femoral arteries documented by Sell et al. [31-33]. Iwasaki et al. found a significantly lower ultimate tensile strength in a bovine aorta; however, these samples were taken from calves 2 to 14 days old, which could explain why they are weaker than the aforementioned arteries. Overall, values of the ultimate tensile strength of native blood vessels ranged from $0.519 \mathrm{MPa}$ to $1.97 \mathrm{MPa}$, while the Elastic Modulus was in the range of 0.16 MPa to $12 \mathrm{MPa}$. The following table summarizes these findings.

Table 1: Summary Table of Documented Tensile Strengths of Native Blood Vessels

\section{Found in a Literature Review}

\begin{tabular}{|c|c|c|c|c|}
\hline \multicolumn{2}{|c|}{ Summary Table: Tensile Strengths of Native Blood Vessels } \\
Vessel & $\begin{array}{c}\text { Direction of } \\
\text { Force }\end{array}$ & $\begin{array}{c}\text { Ultimate Tensile } \\
\text { Strength }\end{array}$ & Elastic Modulus & Reference \\
\hline $\begin{array}{c}\text { Porcine Carotid Artery } \\
\text { Human Atherosclerotic Carotid } \\
\text { Artery }\end{array}$ & Transverse & $1.97 \pm 0.08 \mathrm{MPa}$ & $0.16 \pm 0.007 \mathrm{MPa}$ & Kurane \\
\hline $\begin{array}{c}\text { Human Atherosclerotic Carotid } \\
\text { Artery (Adventitia) }\end{array}$ & Transverse & $1.802 \pm 0.703 \mathrm{MPa}$ & $\mathrm{N} / \mathrm{A}$ & Teng \\
\hline $\begin{array}{c}\text { Human Atherosclerotic Carotid } \\
\text { Artery (Media) }\end{array}$ & Transverse & $1.23 \pm 0.533 \mathrm{MPa}$ & N/A & Teng \\
\hline $\begin{array}{c}\text { Human Atherosclerotic Carotid } \\
\text { Artery }\end{array}$ & Longitudinal & $1.047 \pm 0.536 \mathrm{MPa}$ & N/A & Teng \\
\hline $\begin{array}{c}\text { Human Atherosclerotic Carotid } \\
\text { Artery (Adventitia) }\end{array}$ & Longitudinal & $1.996 \pm 0.867 \mathrm{MPa}$ & N/A & Teng \\
\hline $\begin{array}{c}\text { Human Atherosclerotic Carotid } \\
\text { Artery (Media) }\end{array}$ & Longitudinal & $0.519 \pm 0.27 \mathrm{MPa}$ & N/A & Teng \\
\hline Bovine (Calf) Aorta & Transverse & $0.827 \pm 0.133 \mathrm{MPa}$ & $3.31 \pm 0.56 \mathrm{MPa}$ & Iwasaki \\
\hline Femoral Artery & Longitudinal & $1-2 \mathrm{MPa}$ & $9-12 \mathrm{MPa}$ & Sell \\
\hline
\end{tabular}




\subsubsection{Synthetic Vascular Graft Materials}

Synthetic vascular grafts can be categorized into two classifications, degradable and non-degradable. Non-degradable, synthetic grafts have been used in patients since the 1950's; however, the most common non-degradable materials used in vascular graft applications today are ePTFE and polyethylene terephthalate (PET), also known as Dacron $[1,4,13]$. Dacron is considered a standard vascular graft material for large diameter vessels, and is typically fabricated using a velour technique where fibers are looped together to form a knitted graft, which allows for more compliance and greater tissue ingrowth $[4,16]$. Dacron has very high tensile properties with a reported ultimate tensile strength of 170-180 MPa and an elastic modulus of 14,000 MPa [4, 34]. The extremely large elastic modulus, however, makes this material much more rigid than native vessels causing non-compliance and decreased graft patency, especially in small diameter vessels $(<6 \mathrm{~mm})[1,34,35]$.

The same patency issues have been seen in small diameter ePTFE vascular grafts, however, the material shows excellent patency at five years in lower limb bypass grafts [4]. Many researchers have also shown improved graft healing characteristics by increasing the porosity of ePTFE grafts [34, 36, 37]. While there are published tensile properties of ePTFE grafts, it is difficult to compare tensile strengths due to the variations in testing methods among different institutions [37-39]. For instance, Isaka et al. and Doble et al. use a similar procedure of cutting strips of the ePTFE tube, but use different testing speeds as well as different sample preparation techniques. Doble et al. washed all adhered tissue and lipids off of the graft before testing whereas Isaka et al. only noted that the fluoroethlyenepropylene filament originally reinforcing the graft was removed 
prior to testing $[37,39]$. It should also be noted that most grafts $(52 \%)$ studied by Doble et al. were tested 12-24 months following implant [39]. Catanese also uses a similar method to compare the tensile properties of medical grade ePTFE tubes with varying intermodal distances [40]. Overall, these various testing methods have provided ultimate tensile strengths of ePTFE tubing ranging from 3.9 MPa to 17.2 MPa. The Elastic Modulus of this data ranges from 47.1 MPa to 59.3 MPa. The following table shows the results as recorded by the aforementioned studies. 
Table 2: Summary Table of Documented Tensile Strengths of ePTFE Tubing Found in a Literature Review

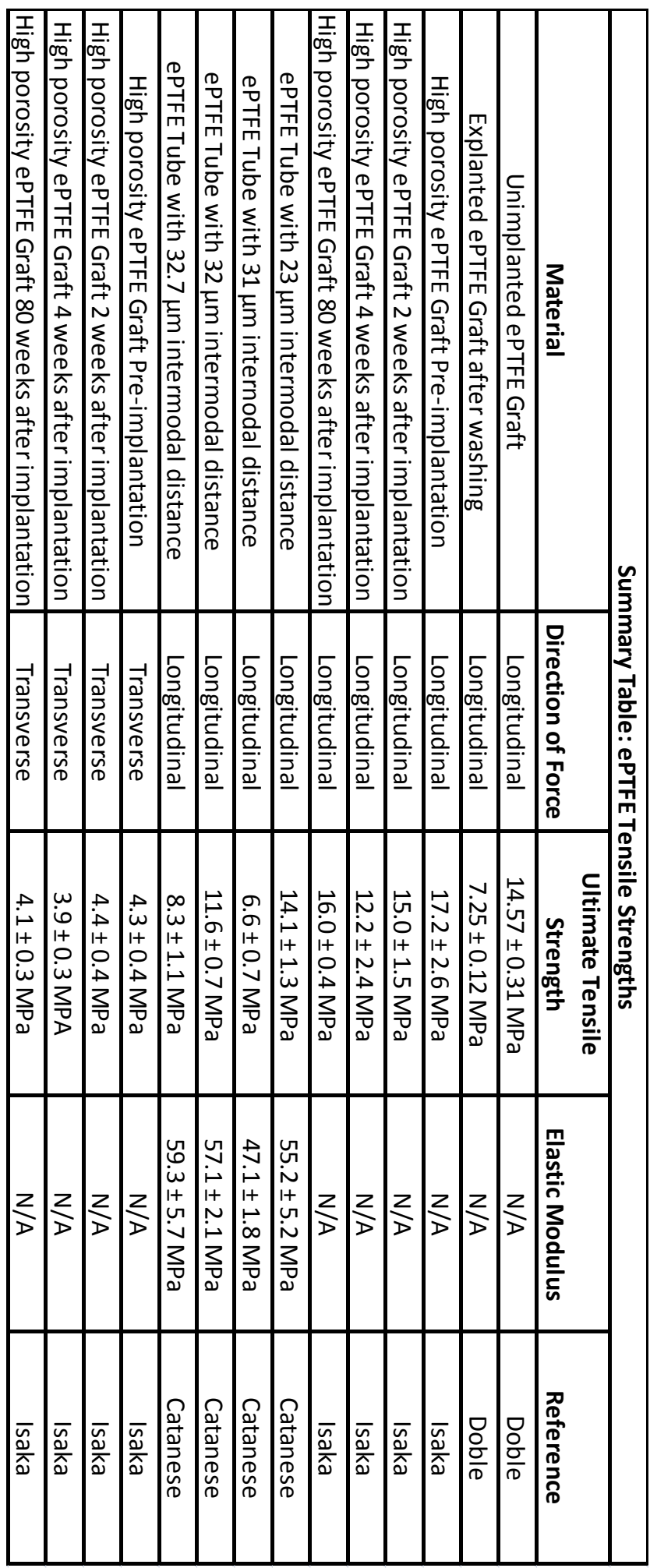




\subsubsection{Bioresorbable Vascular Graft Materials}

Many biodegradable, or bioresorbable, materials have been studied for small diameter arterial replacement $[9,18,33,41]$. An ideal degradable graft or scaffold should degrade at the same rate at which new tissue forms [42]. While PLGA is a common graft material, other biodegradable materials such as poly(glycerol sebacate) (PGS), polyglycolic acid (PGA), poly-L-lactic acid (PLLA), polydioxanone, and polyglyconate (Maxon) have also been used [9, 11, 18, 33, 41-43]. In native arteries, collagen provides stiffness to the vessel while the elastin contributes to the elasticity [11]. In order to achieve mechanical properties similar to native vessels, degradable grafts will sometimes be combined with either collagen or elastin $[11,18]$. While the tensile properties of a synthetic material may be higher than native vessel properties to begin with, adding elastin can greatly reduce the tensile strength to make it more compliant with native vessels $[11,33]$. It should be noted that tensile properties can greatly vary along the course of degradation [42]. Furthermore, certain sterilization techniques, as shown by Shearer et al, can reduce tensile properties of degradable scaffolds [43].

Overall, the ultimate tensile strength of biodegradable materials ranges from $0.24 \mathrm{MPa}$ to 14.46 MPa, while the Elastic Modulus of these tubes ranges from 2.45 MPa to 580.2 MPa. The following table summarizes the tensile properties of bioresorbable materials found in a literature search. 
Table 3: Summary Table of the Documented Tensile Strengths of Bioresorbable Graft Materials Found in a Literature Review.

\begin{tabular}{|c|c|c|c|c|c|c|c|c|c|c|c|c|c|}
\hline 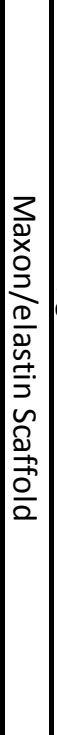 & 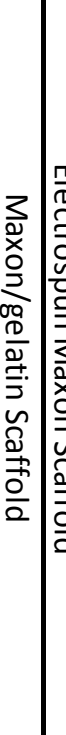 & 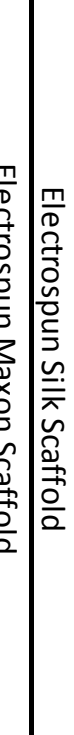 & 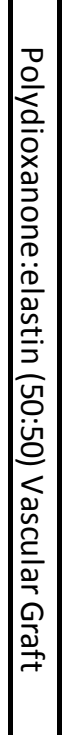 & 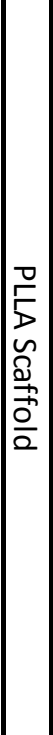 & 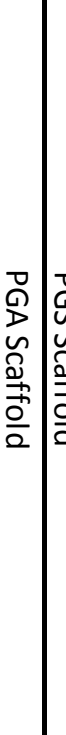 & 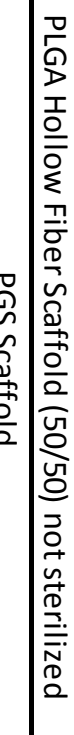 & 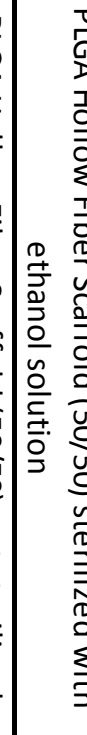 & 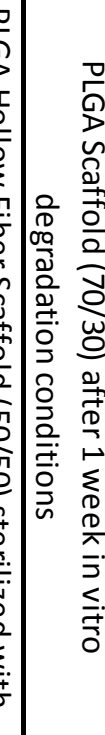 & 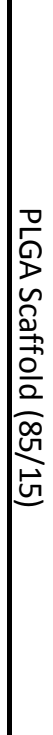 & 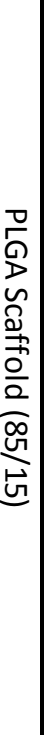 & & & \\
\hline & 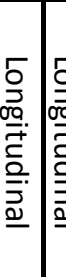 & & 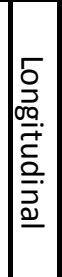 & 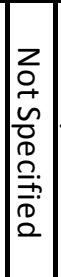 & 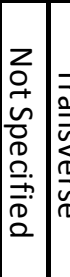 & 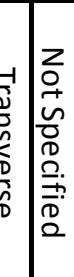 & 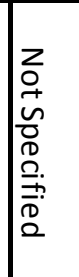 & | & 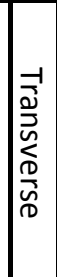 & $\underline{\underline{D}}$ & & & 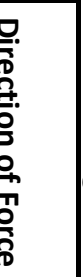 \\
\hline 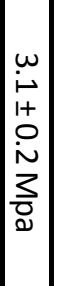 & 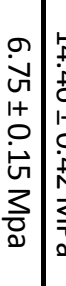 & 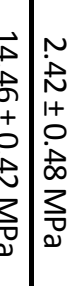 & 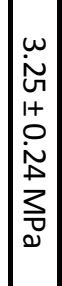 & 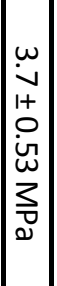 & 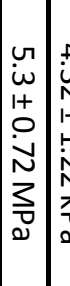 & & $\mid \begin{array}{l}\vec{y} \\
\text { y. } \\
3 \\
0 \\
0\end{array}$ & 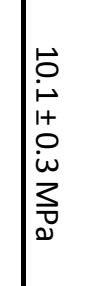 & 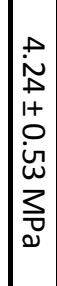 & 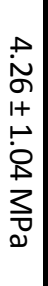 & $\begin{array}{c}0 \\
\\
1+ \\
0 \\
0 \\
0\end{array}$ & $\begin{array}{l}0 \\
\text { ज } \\
+ \\
0 \\
\vdots \\
0 \\
0 \\
0\end{array}$ & 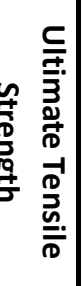 \\
\hline & 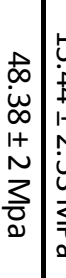 & 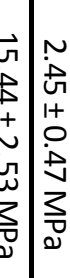 & $\mid \begin{array}{c}0 \\
0 \\
8 \\
1+ \\
1+ \\
0 \\
2 \\
2 \\
3 \\
0 \\
0\end{array}$ & 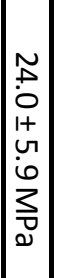 & 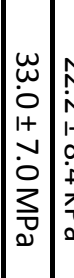 & 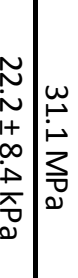 & 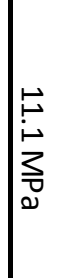 & 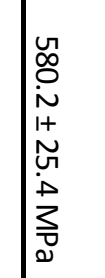 & 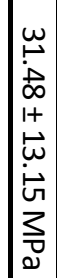 & $\vec{E}$ & & & 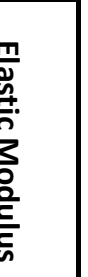 \\
\hline & 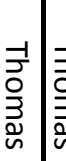 & 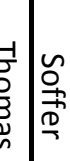 & 1 & 10 & गु) & 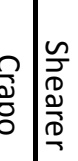 & 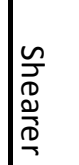 & 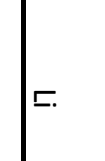 & & & & & \\
\hline
\end{tabular}




\subsection{Testing the tensile properties of tubular constructs}

The most common cause of long term failure of small diameter vascular grafts is intimal hyperplasia $[2,17]$. Although the cause of this condition is not clearly understood, it has been shown that disturbed blood flow or injury to a vessel is closely associated with this condition. Because vascular grafts have different elastic properties than the native vessel next to which they are implanted, this leads to fluctuations in the shear stress at the endothelium $[2,34]$. Along with the injury due to suturing, the blood vessel prostheses have very little chance of lasting long periods of time without the event of hyperplasia [2]. This creates a need for vascular prostheses, especially in small diameter vessels, to have tensile properties close to or matching native vessel properties.

Two methods used to determine the mechanical properties of tubular materials are a burst pressure test or a tensile test. A burst pressure test applies internal pressure to a tube until the construct breaks. A material's burst pressure is measured in mmHg. This test requires special equipment and a rather long sample of tubing [44]. About half of all literature reviewed for this thesis used burst pressure to characterize the mechanical properties of vascular grafts. Because Cal Poly’s campus does not have the proper equipment to perform a burst test for ePTFE and PLGA tubing, the focus of this thesis will be using a tensile testing machine.

A tensile test is one of the most basic methods for determining the mechanical behavior of a material [45]. During a tensile test, a load is applied at a constant rate so that the material sample is pulled until it fractures. In a typical tensile test, the material sample is cut into the shape of a dog bone with a fixed cross-sectional area and placed 
between two grips on a universal testing machine, as shown in the figure below. A universal testing machine, also known as an electromechanical testing system, is an automated test machine commonly used for tension and compression tests [45]. One grip remains static, while the other grip pulls on the material as the force is applied [8]. The purpose of the dog bone shape in the material test sample is to create a notch in order to focus the deformation of the material to the pre-set gage length, which is the length of the sample in between the grips where deformation should occur [44]. This also prevents the material from breaking at the grips.

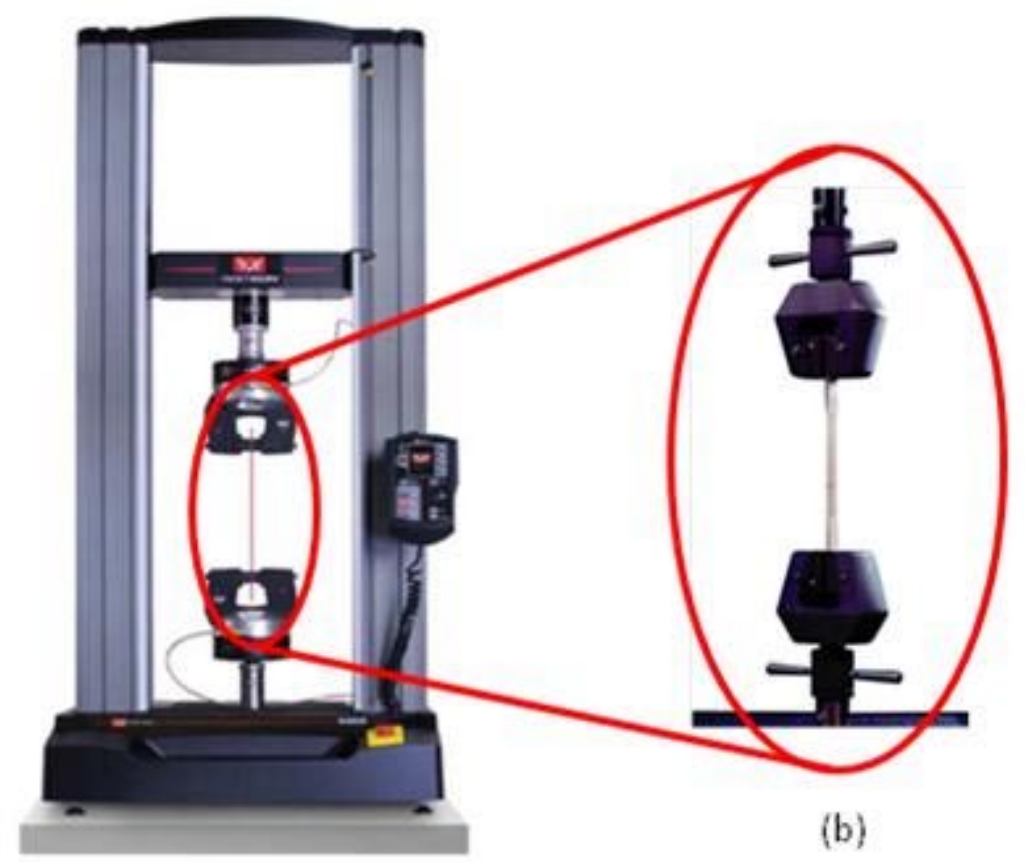

(a)

Figure 4: (a) Universal testing machine, (b) magnified view of grips holding dog bone shaped test sample [45].

The following sections will describe different methods used to test the longitudinal and transverse tensile properties of tubular constructs including available 
standards as well as experimental testing methods used in biomedical applications or other industries.

\subsubsection{Longitudinal tensile testing of tubular constructs}

For longitudinal testing of tubular structures, ASTM standard D 638 (Volume 08.01 ) outlines specific methods for testing the tensile properties of normal dumbbell-shaped plastics along with plastic tubes and rods.

For sheet, plate, and molded plastics, this standard categorizes a test specimen by its physical characteristics, such as rigidity, and assigns the specimen to a type I, II, III, or IV based on the thickness of the test sample. Based on the material type, specific dimensions for the test specimen are assigned to the material sample as shown below. 


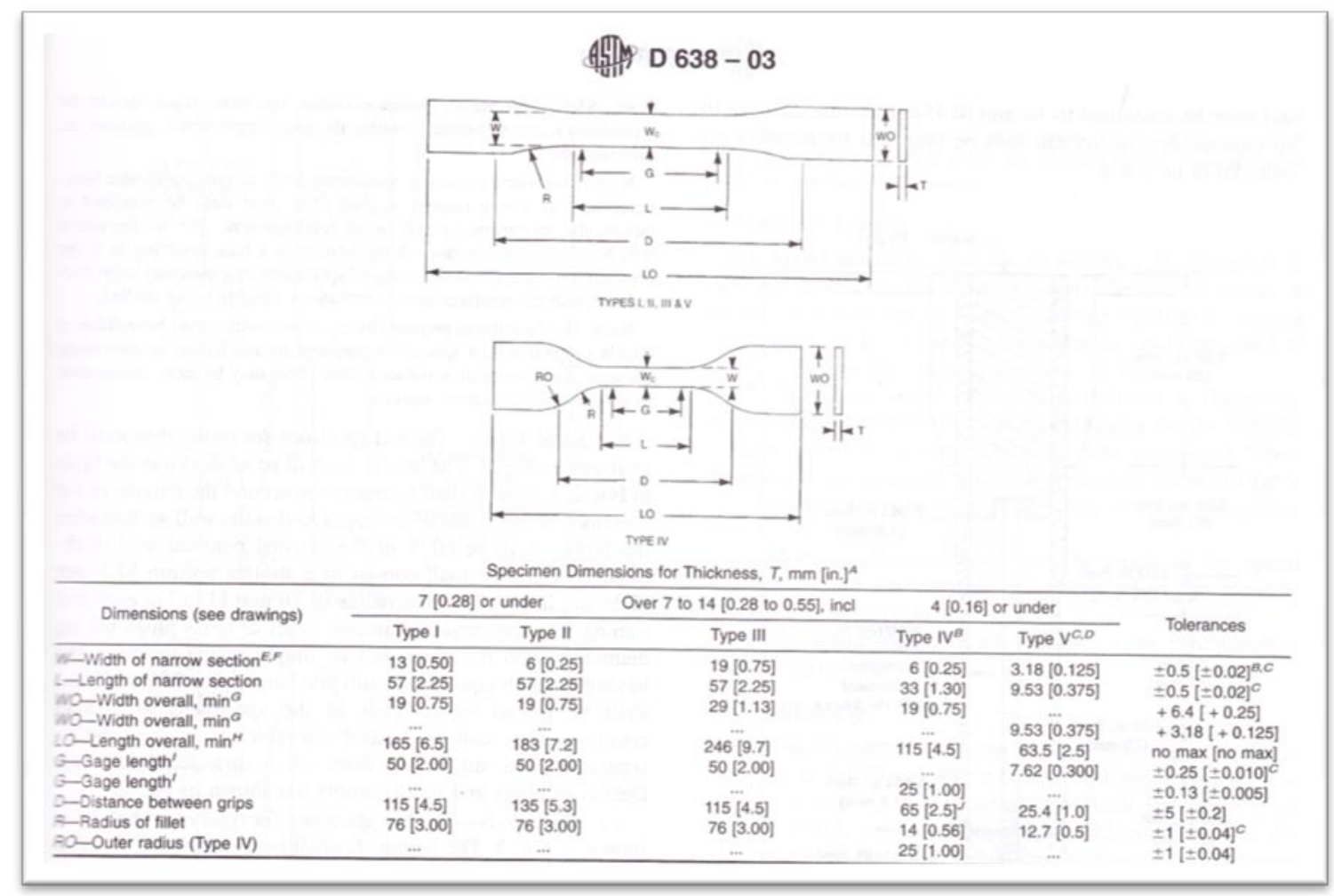

Figure 5: ASTM D 638 outlines dimensions for different types of materials to be tensile tested in the longitudinal direction. Based on the category a specific material fits into, this table shows how the test sample should be prepared [46].

The test apparatus is clearly described as consisting of a testing machine, fixed member, moveable member, grips, drive mechanism, load indicator, crosshead extension indicator, and extension indicator. It should be noted that tensile properties differ among test samples based on the method of material preparation, such as cleaning, coating, cutting, or submersing the material in fluid before testing. Thus, when comparing tensile properties among materials, the test samples to be compared must be prepared in the same way. 
For plastic tubes, another table referenced in ASTM D 638 is used to determine the required dimensions of the test sample based on the length of the specimen. To keep the shape of the tubular material, metal plugs are to be fitted tightly into both ends of the test sample as diagrammed in the figure below. The standard suggests using steel or brass for these plugs. A similar table is used for plastic rods, and the method of testing is the same, but without the metal plugs. It is important to note that for both rods and tubes, this standard only specifies instructions for rigid materials. Biomaterials used for vascular grafts are usually very pliant, and there is no ASTM standard that covers the tensile testing of non-rigid plastic tubes [46].

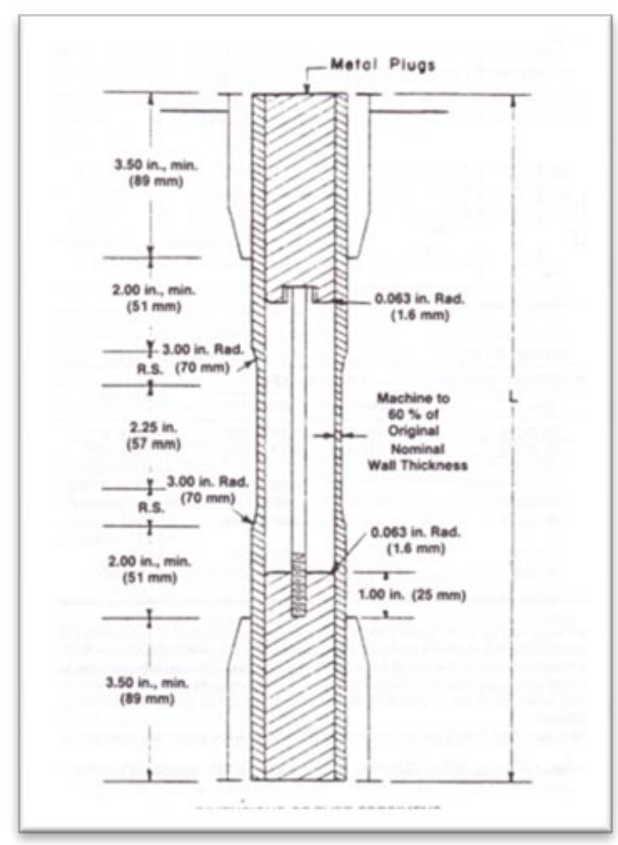

Figure 6: ASTM D 638 diagram shows metal plugs, shaded here with diagonal lines, to hold the shape of the plastic tube while testing for longitudinal tensile properties [46]. 
The International Organization for Standardization (ISO) also offers a standard for testing the longitudinal properties specifically for tubular vascular prostheses. Titled “Cardiovascular implants-Tubular vascular prostheses”, ISO 7198 describes a fairly vague procedure on how to determine the longitudinal tensile strength of small diameter tubular materials. The standard does state that the prostheses should stay in tubular form, but does not specify how the ends of the tube should be gripped in the universal testing machine's jaws. ISO 7198 also states that the longitudinal tensile strength should equal the maximum load placed on the test sample and should be expressed in kilonewtons. This is different than any of the other standards or literature found that mostly express tensile strength in pascals (for a tensile test) or mmHg (for a burst pressure test) [47].

Current methods used to test the longitudinal properties of vascular grafts are varied; however, most research found as a preface to this thesis follows suit to ASTM standard D 638 in testing a strip of material either in a dog bone or rectangular shape [11, 32, 33, 37-39, 48, 49]. Few researchers document any standards in their testing, but Doble et al. mentions performing tensile testing on explanted ePTFE vascular grafts as per ASTM D 882, the standard test method for testing the tensile properties of thin plastic sheeting [39]. Similarly, Guan et al. references ASTM D 638 in their testing; however, do not provide specific details as to the size or shape of their test samples [50].

Most researchers testing longitudinal tensile properties of tubes used Instron testing machines to test their samples $[32,37,39,50]$. Thomas et al. used a different instrument, called a dynamic mechanical analyzer, to apply a cyclic load instead of a steadily increasing force as performed on Instron tensile testing machines [11]. Two 
other studies used tensile machines from a competitor of Instron Corporation, MTS Systems $[33,48]$.

Catanese et al. were the only researchers that described testing medical grade ePTFE tubes in their tubular form using an Instron 5583. The description of the method used is very vague, only noting the ePTFE tube samples were placed in plastic tubing to help with friction and avoid tearing at the edge of the steel wedge grips of the machine [40]. The following table summarizes the methods found in the literature testing the longitudinal tensile properties of vascular graft materials or native vessels. 
Table 4: Summary Table of the Longitudinal Testing Methods of Tubular

Constructs Found in a Literature Review.

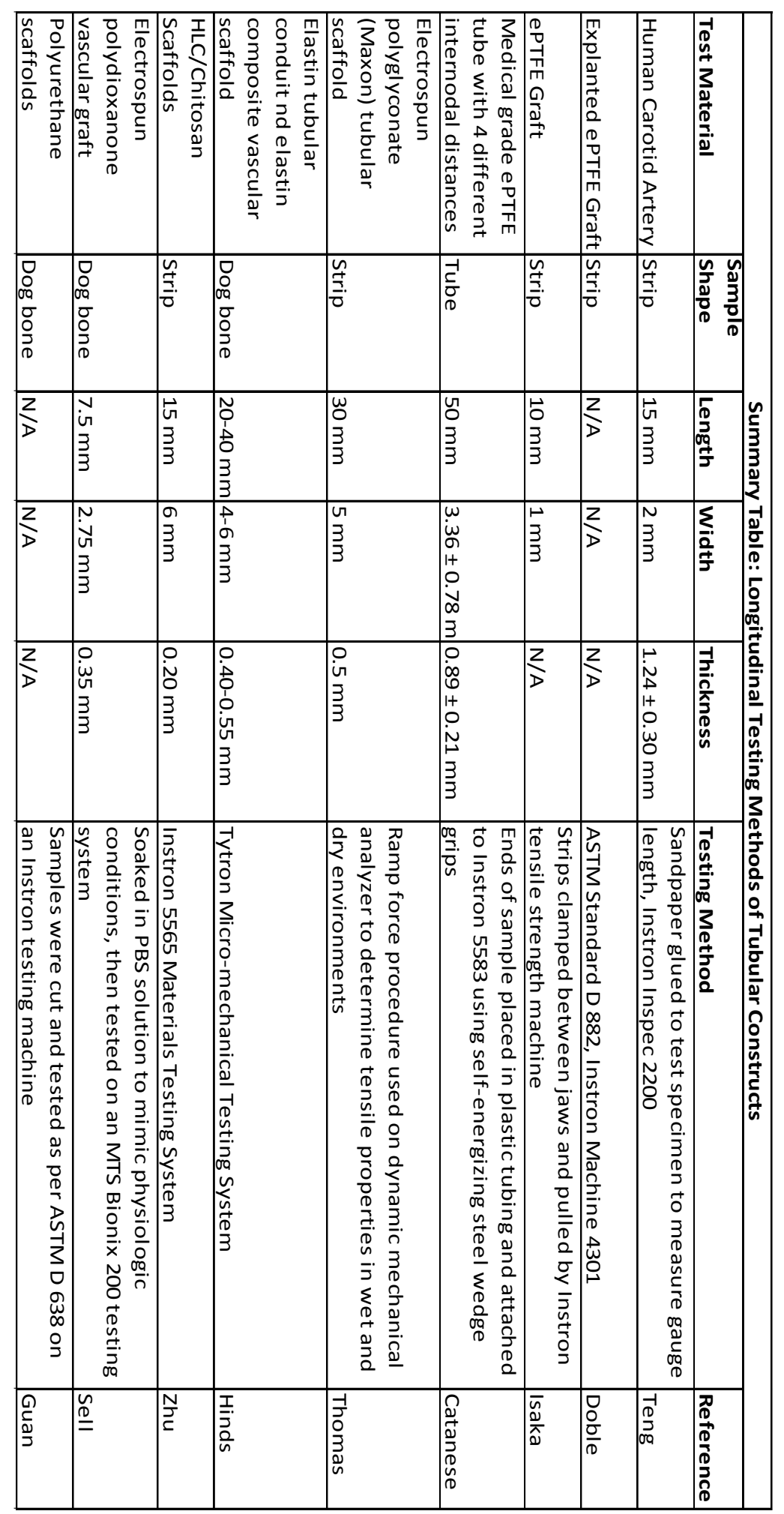




\subsubsection{Transverse tensile testing of tubular constructs}

Testing the transverse tensile properties of a polymeric tube requires different test methods and fixtures than longitudinal tensile tests. ASTM standard D 2290 (V 08.04) describes methods to test the hoop tensile strength of plastic pipes, and is applicable to reinforced-thermosetting resin pipe and extruded or molded thermoplastic pipes of any size. The small diameter of the plastic tubes used in biomedical applications can make this method of testing difficult to perform; thus, efforts in the field of biomedical engineering are exploring novel ways of tensile testing these materials, a few of which will be discussed in this section.

ASTM standard D 2290 (V 08.04), titled "Standard Test Method for Apparent Hoop Tensile Strength of Plastic or Reinforced Plastic Pipe by Split Disk Method”, offers a detailed procedure and description of the materials needed to test the hoop tensile strength of a tubular plastic material. Hoop tensile strength refers to the transverse tensile strength of metal or plastic piping. A universal tensile testing system similar to that described in ASTM D 638 is required along with a specific test fixture to test the abnormal geometries of plastic piping. This fixture, shown below, consists of two Dshaped blocks that are fit into the cross-section of a test specimen and subsequently pulled apart by the applied load during the test. 


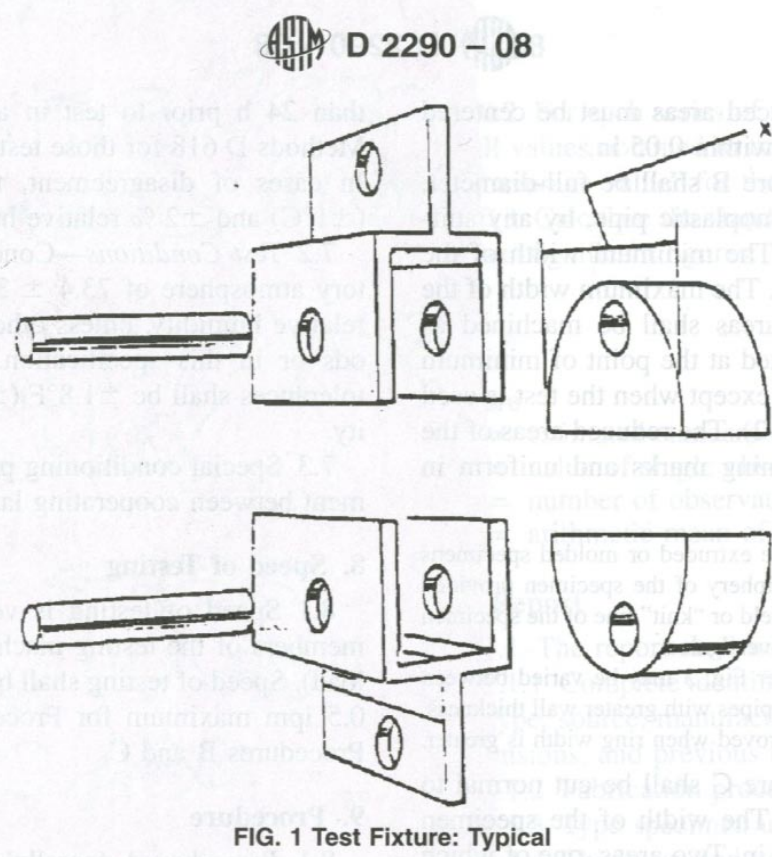

Figure 7: Diagram of fixture specified to test the hoop tensile strength of plastic tubing according to ASTM Standard D 2290. D shaped blocks, shown to the right, are inserted into the tubular test sample then attached to the fixture with pins shown to the left [51].

Based on the size and type of plastic being tested, three different procedures are outlined in the ASTM standard describing the width between the two disks, the size of the test specimen, and the speed at which the test must be performed. Procedure A requires a minimum overall width of 0.90 inches with a reduced section minimum width of 0.55 inches for the test sample. For Procedure B, the width of the sample must be between 0.50 and 2.00 inches, and Procedure $\mathrm{C}$ states the width of the sample should be between 1.75 and 2.00 inches. The testing speed for Procedure A is between 0.1 and 0.5 inches per minute, while Procedures B and C require a speed of 0.5 inches per minute. For a thermoplastic pipe of any size, Procedure B is recommended. The following figure 
and table specifies the guidelines of using this procedure, however, the full ASTM D 2290 standard can be found in Appendix A.

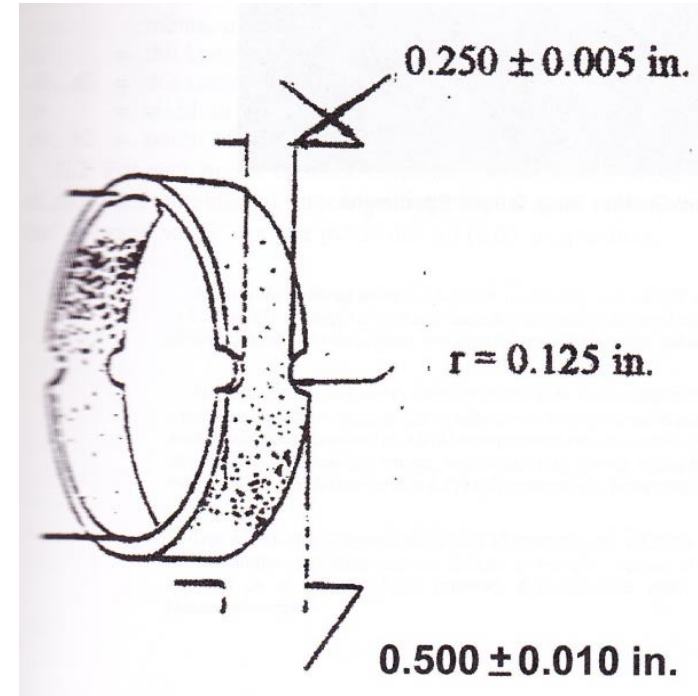

FIG. 3 Reduced Section Specimen for Procedure B

Figure 8: This figure, taken from ASTM D 2290, shows the dimensions of a prepared tubular sample ready to tested using the split disk method described in the standard [51]. 
Table 5: Dimensions specified by ASTM D 2290 to prepare a material sample to be tested in tubular form using the split disk method [51].

\begin{tabular}{|c|c|}
\hline \multicolumn{2}{|c|}{$\begin{array}{l}\text { Guidelines for testing thermoplastic piping using split disk } \\
\text { method as specified by ASTM Standard D } 2290 \text { (V 08.04) }\end{array}$} \\
\hline Width of split disk & 0.50 in. +0.05 in. \\
\hline Width of test specimen & 0.50 in. +0.01 in. \\
\hline Width of reduced test section & 0.25 in. +0.005 in. \\
\hline Speed of testing & $0.50 \mathrm{in} . / \mathrm{min}$. \\
\hline
\end{tabular}

ISO 7198 also describes a method of testing the transverse, or circumferential, tensile strength specifically for tubular vascular prostheses. In this standard's methods, it indicates that the sample in its tubular form shall be placed onto two rounded pins that are subsequently stretched by a unidirectional load of a tensile testing machine. ISO 7198 does not specify the size of the pins or holders that should be used; however, it does mention that the pins should be appropriately sized for the sample prosthesis to be held. Appendix B shows ISO 7198 in its entirety, including the diagram that illustrates the pins and holder that should be used in relation to the tensile force applied from the universal testing machine. Similar to the ISO 7198 method to test the longitudinal tensile strength of vascular prostheses, this portion of the document denotes the circumferential tensile strength as the maximum load divided by two times the length of the original sample. 
This is expressed in kilonewtons per millimeter, which is, again, different than any other researched standards or literature [47].

Researchers have documented numerous ways of tensile testing tubular materials of all different sizes, properties, and characteristics [5, 32, 44, 52, 53]. Arsene et al, Krashchenko et al, and Wang et al. offer methods of testing the transverse properties of structural tubing, reinforced plastic tubing, and hydroformed tubes, which could all be potentially applied to small diameter tubular applications as well $[44,52,53]$.

Krashchenko et al. and Wang et al. both describe the split disc method similar to ASTM D 2290, but Wang et al. specifies their method for hydroforming tubes smaller than 152.5 $\mathrm{mm}$ in diameter $[52,53]$. This split disk method can be summarized by placing two Dshaped blocks inside the tubular test sample, then pulling the blocks apart in a uniaxial direction. This method is illustrated in the figure below [53].

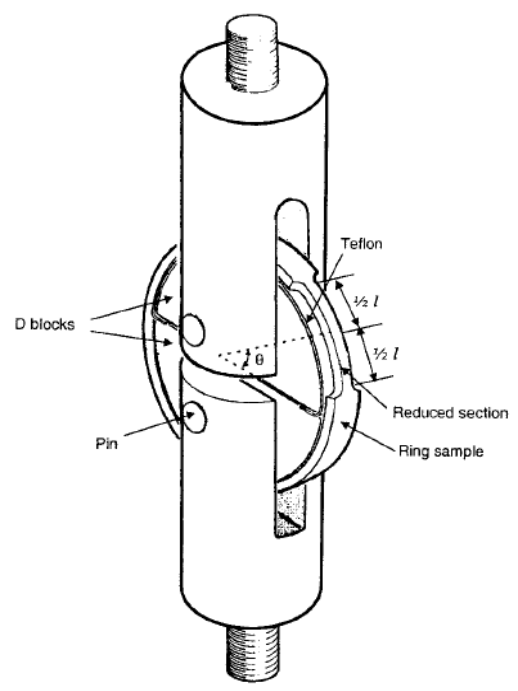

Figure 9: Split disk method of testing the tensile properties of a tubular specimen [53]. 
Arsene et al. developed a novel ring method for structural tubing that addresses some limitations to the split disc apparatus, specifically the non-uniform distribution of strain in the material sample and the stress concentration between the two half cylinder disks. The new design consisted of three parts, one of which is placed in the middle of the sample cross-section with the same diameter as the sample. The central piece, placed in the middle of the cross-section, helps reduce the bending moments in the sides of the tubular sample that sometimes occurs with the split disk method. Two other blocks were placed above and below the central piece, as shown below, that are pulled in the direction of the applied load [44].
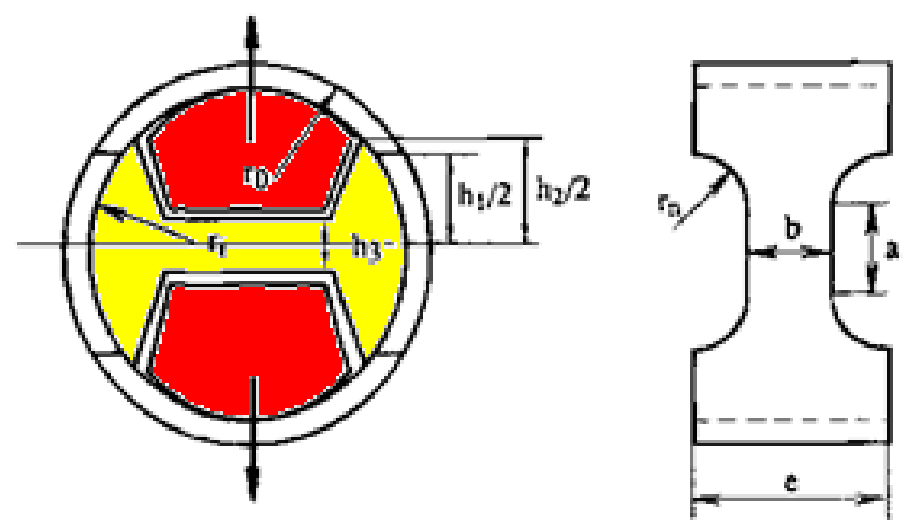

notched ring specimen

Figure 10: Novel ring test method to determine transvers properties of tubular specimens. Yellow highlighted area shows center piece that reduces bending moment in sample. Red shapes illustrate end pieces within sample where the uniaxial force is applied [44].

The rising presence of tissue engineering in the biomedical field has caused improved transverse tensile testing methods for small diameter tubes to emerge in academic journals and research studies. Berglund et al, Seliktar et al, Crapo et al, and 
Soffer et al. describe a ring test method used to test small diameter tubes related to tissue engineering $[5,10,41,54]$. The ring test method can be summarized as using two hooks or rods placed inside of the tube sample and pulled apart in the direction uniaxial to the transverse direction of the tube. The following figure diagrams this method.

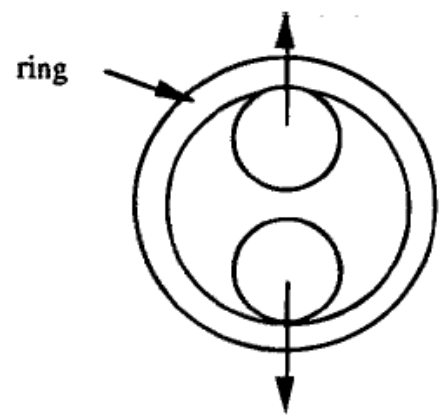

Figure 11: Typical ring test method using to hooks or rods to pull apart ring, or tube, sample[44].

Berglund et al. tested the viscoelastic properties of tissue engineered blood vessels made from fibroblasts and type I collagen using a test fixture developed by Seliktar et al. [5, 54]. This fixture held the tissue engineered blood vessel between two hooks that were pulled apart to apply unidirectional loading. To measure the true strain of the vessel, four black beads were placed along the cross-section, two on each wall of the sample, as shown in the figure below [54]. Similar test methods were used by Crapo et al. and Soffer et al to test the transverse properties of PGS scaffolds and silk-based scaffolds, respectively; however, neither mentioned beads placed along the test sample [10, 41]. 

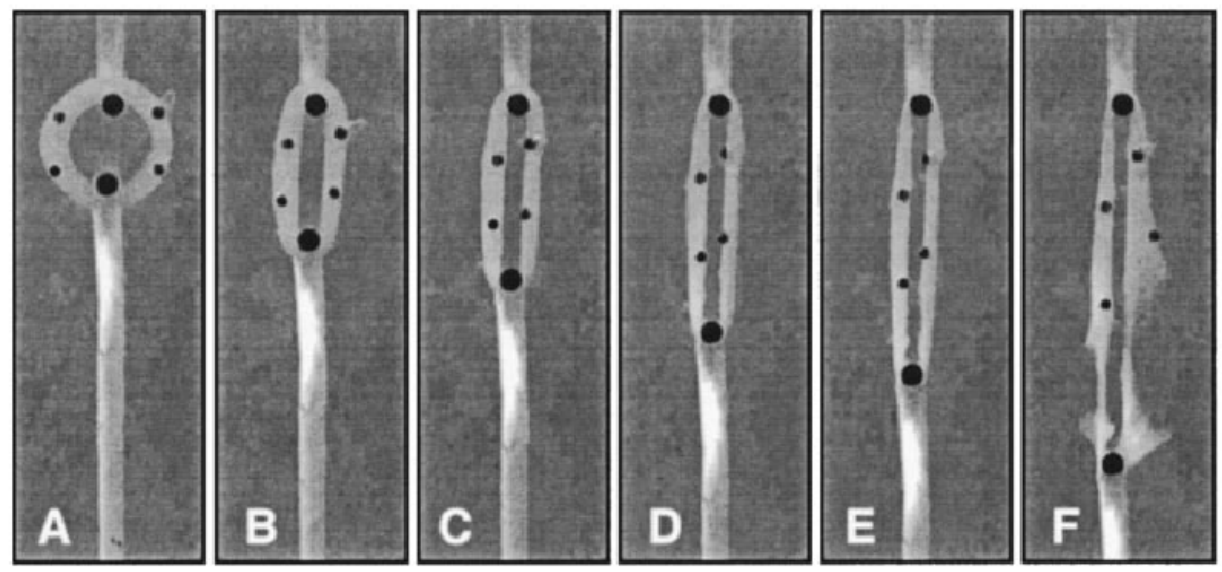

Figure 12: Ring test used to determine transverse tensile properties of tissue engineered blood vessels. Large black dots show the hooks pulling apart the specimen. Smaller black dots show the displacement of the sample over the course to the test [54].

Another commonly used method reported for testing the transverse tensile properties of vascular graft or tissue engineered blood vessel material is to use flat pieces of the material; however, this method may not produce as accurate of results as the ring test method. This method involves cutting the tubular sample, thereby creating a test strip by which to test in the transverse direction on a universal testing machine [3, 32, 37]. This method of testing is also currently used to test the transverse tensile properties of PLGA and ePTFE tubes on Cal Poly's campus. The following pictures show a piece of PLGA before and during a tensile test using Cal Poly’s Instron 2200 In-Spec testing machine. 


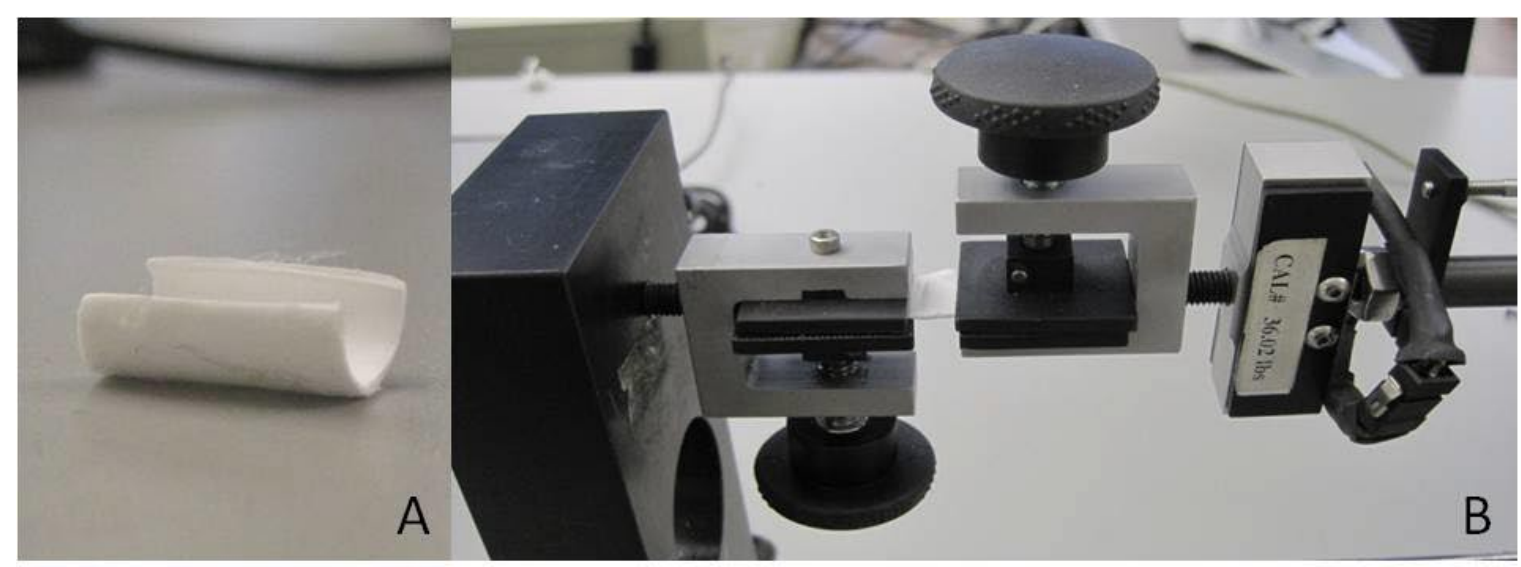

Figure 13: A) A piece of PLGA prior to tensile testing, cut down the length of the tube. B) The sectioned piece of PLGA during a tensile test using the current method of testing at Cal Poly.

The following table summarizes the previously mentioned methods of transversely testing vascular graft materials. 
Table 6: Summary Table of the Transverse Testing Methods of Vascular Graft Constructs Found in a Literature Review

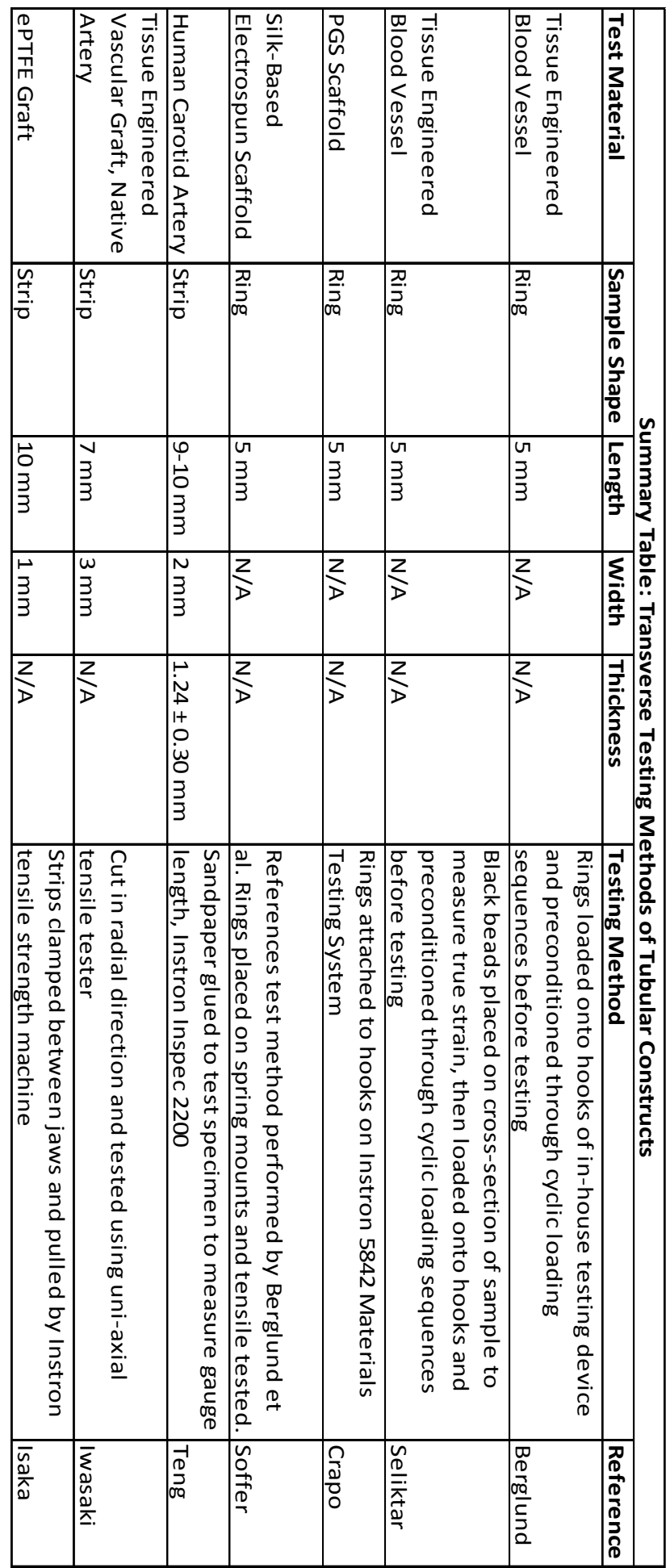




\subsection{Summary and Aims of the Thesis}

Today, eight million Americans are affected by peripheral artery disease alone, and millions more suffer worldwide, creating an enormous need for continued research in treatment and prevention [30]. This provides a huge potential for further research to expand the biomaterials available for vascular graft applications. As discussed earlier, synthetic vascular grafts such as Dacron and ePTFE have been shown to be suitable for large diameter vessels, but have not been successful in smaller vessels. While materials such as PLGA show promise in small diameter vascular grafts, more efforts must be made to improve their biocompatibility as well as mechanical properties. Because the long term effects of implantation are still unknown, further investigation is required, and other material modifications may provide more desirable results [9]. It is clear that mechanical properties have a major impact on the suitability of a material for a specific biomedical application, and there is currently limited documentation of tensile testing methods for small diameter tubular biomaterials. Once an accurate industry standard of testing transverse and longitudinal properties of vascular constructs has been developed, researchers will be able to more accurately compare different biomaterial properties, which in turn will lead to more efficient advancement in biomedical design applications.

It is the aim of this thesis to develop and implement a method of testing the longitudinal and transverse tensile properties of small diameter tubular constructs on Cal Poly's campus. The primary deliverable at the end of this thesis will be two test fixtures that can attach to the Cal Poly Biomedical Engineering department's Instron tensile testing machine located on campus. In addition, a secondary deliverable will be tensile property data of small diameter tubular constructs gathered using the completed test 
fixtures. Because of the use of ePTFE and PLGA tubular constructs in Cal Poly's laboratories, these materials will be used to validate the completed design. This thesis will follow the design process from ideation to implementation, and provide sufficient documentation to suggest a standard method of testing tensile properties of small diameter tubular constructs. 


\section{Chapter 2-Tensile Testing Capabilities on Cal Poly's Campus}

This chapter will provide an overview of all tensile machines available on Cal Poly's campus from the Biomedical, Mechanical, and Materials Engineering departments. A brief description of each machine will be provided along with machine specifications, the location of each machine, and machine service fees from each department.

\subsection{Biomedical Engineering Department}

The Biomedical Engineering department has one tensile testing machine on campus, an Instron In-Spec 2200. This machine is housed in Building 192 Room 330; however, it is portable and can be easily moved to other lab spaces. The Instron In-Spec 2200 has the following specifications:

Table 7: Specifications for Instron In-Spec 2200 located in Building 192-330 on Cal Poly's campus.

\begin{tabular}{|l|l|}
\hline Instron In-Spec 2200 & Specification \\
\hline Load cell capacity & $225 \mathrm{~N}(50 \mathrm{lb})$ \\
\hline Maximum force & $500 \mathrm{~N}(110 \mathrm{lb})$ \\
\hline Speed range & 1 to $750 \mathrm{~mm} / \mathrm{min}$ \\
\hline Height & $460 \mathrm{~mm}$ \\
\hline Width & $245 \mathrm{~mm}$ \\
\hline Depth & $230 \mathrm{~mm}$ \\
\hline
\end{tabular}




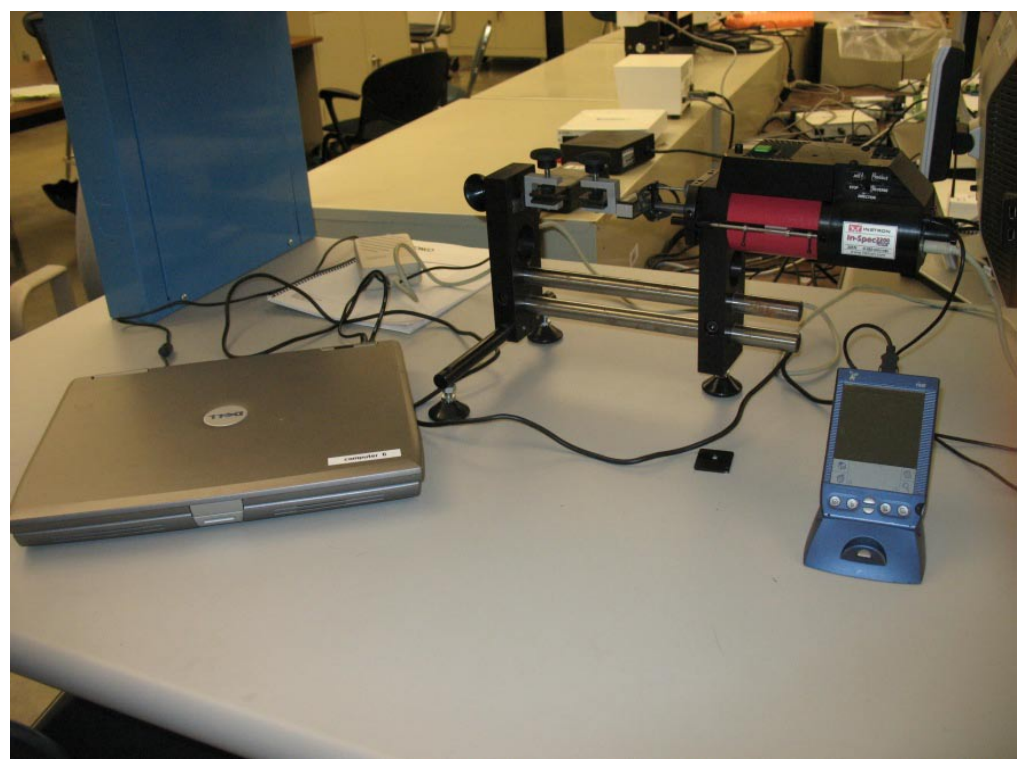

Figure 14: Instron In-Spec 2200 located in 192-330.

This machine is used for mechanical testing of materials mainly within the context of the biomedical field and is part of the curriculum for BMED 420:

Biomaterials. In this class, students test tubular samples of expanded polytetrafluoroethylene (ePTFE) and poly(lactic-co-glycolic acid) (PLGA) in hopes of comparing the elastic modulus and ultimate tensile strength of their samples in the transverse and longitudinal directions. Samples are cut in rectangular specimens to be tested. A unique characteristic of the In-Spec testing machine is its ability to be positioned horizontally or vertically on a table top or flat surface, and can also perform compression along with tensile tests. The data acquisition system for this machine is a Personal Digital Assistant (PDA) which is set up to a laptop computer so gathered data is easily transferred to an excel spreadsheet. 


\subsection{Materials Engineering Department}

The Materials Engineering department has two tensile testing machines, one of which is an Instron 3369 with a tabletop frame, located in Building 41 Room 201. The Instron 3369 has the following specifications:

Table 8: Specifications for Instron 3369 located in Building 41-201 on Cal Poly’s

Campus.

\begin{tabular}{|l|l|}
\hline Instron 3369 & Specification \\
\hline Load cell capacity & $50 \mathrm{kN}(11250 \mathrm{lb})$ \\
\hline Maximum force & $25 \mathrm{kn}($ at maximum speed) \\
\hline Speed range & 0.005 to $500 \mathrm{~mm} / \mathrm{min}$ \\
\hline Height & $1582 \mathrm{~mm}$ \\
\hline Width & $756 \mathrm{~mm}$ \\
\hline Depth & $707 \mathrm{~mm}$ \\
\hline
\end{tabular}




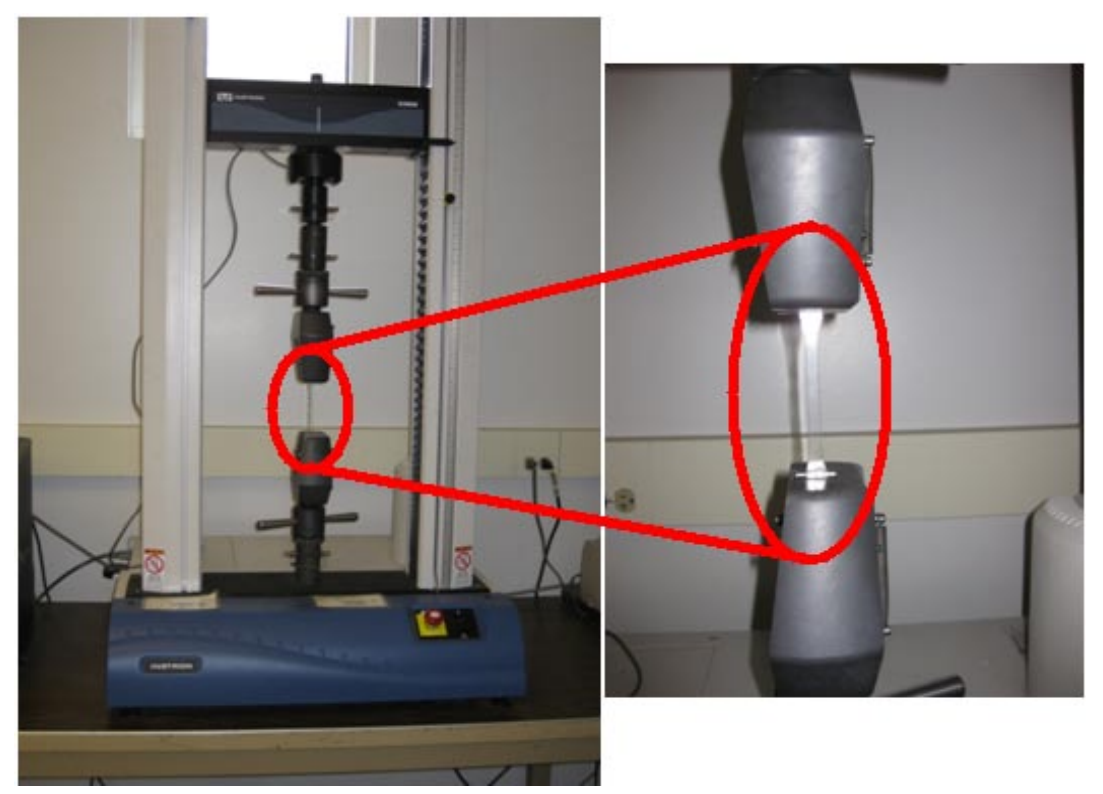

Figure 15: Instron 3369 located in 41-201.

This machine is mainly used as part of the curriculum for MATE 215, which is an introductory materials laboratory in which students test the properties of metals, composites, polymers, etc. Most test specimens that are used are standard dog-bone shaped test samples, as opposed to complex shapes or structures such as tubing or devices. Bluehill 2 software is the data acquisition software used with this machine which makes it easy to select a test method, gather data, and save results either in a report or graph format.

Located in Building 192 Room 210, the Materials Engineering second tensile tester is an Instron 5584. This machine has a floor standing frame with the following specifications: 
Table 9: Specifications for Instron 5584 located in Building 192-210 on Cal Poly's campus.

\begin{tabular}{|l|l|}
\hline Instron 5584 & Specification \\
\hline Load cell capacity & $150 \mathrm{kN}(33000 \mathrm{lb})$ \\
\hline Maximum force & $110 \mathrm{kN}$ (at maximum speed) \\
\hline Speed range & 0.001 to $750 \mathrm{~mm} / \mathrm{min}$ \\
\hline Height & $2092 \mathrm{~mm}$ \\
\hline Width & $1300 \mathrm{~mm}$ \\
\hline Depth & $756 \mathrm{~mm}$ \\
\hline
\end{tabular}

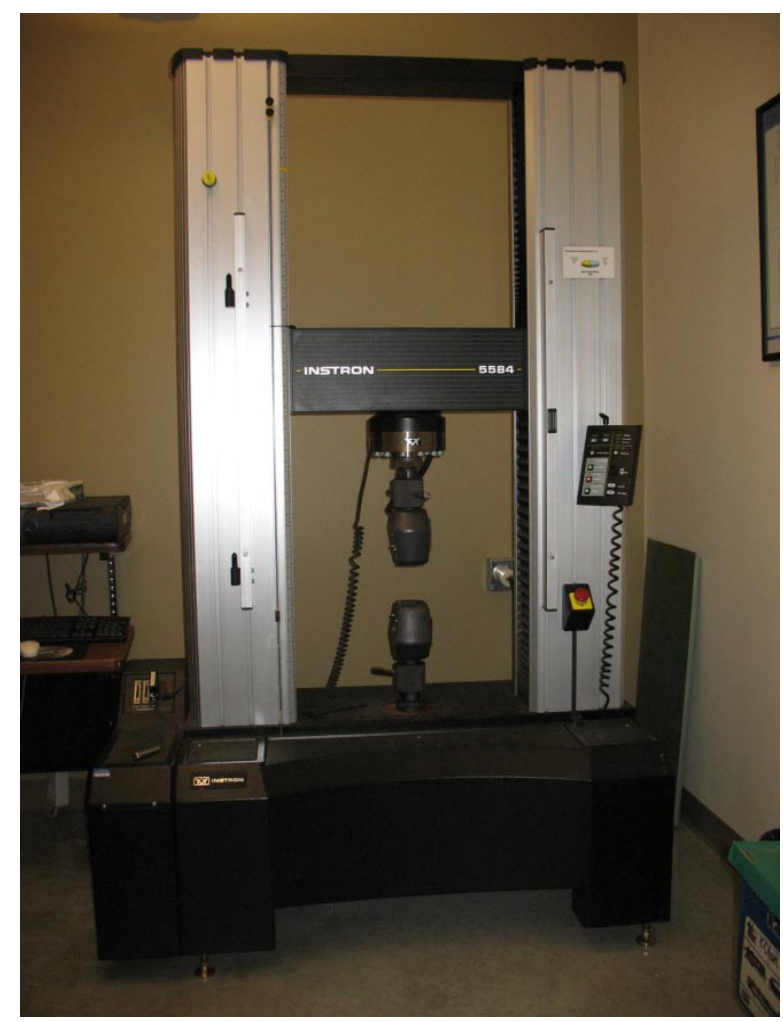

Figure 16: Instron 5584 located in 192-210. 
Mainly utilized by upper division laboratories and student projects, this Instron tests a variety a materials including polyethylene, aluminums, steels, alloys, and polymers. Data is gathered using a Digital Signal Processor (DSP) with a user-friendly interface. As the data is being gathered and transferred to the connecting computer, the user can see real-time results using the Bluehill 2 software.

The Materials Engineering department charges \$21/hour for Cal Poly students, faculty, and staff to use the Instron equipment; however, for non-Cal Poly users, the fee increases to $\$ 30 /$ hour.

\subsection{Mechanical Engineering Department}

The Mechanical Engineering department also has two tensile testing machines located in Building 192 Room 135, which is the Mechanical Engineering composites laboratory. Both machines are Instron tensile testing machines, one with a floor standing frame and another with a table top frame. The floor standing frame is an Instron 1331 and is hydraulically driven. The table top frame, on the other hand, is an Instron 4400R and is electrically driven. Both machines are used as part of the curriculum for ME 410, ME 328, and ME 412. While the Mechanical Engineering department does not have specified fees involved with use of these machines, inquiries to use either Instron can be emailed to me-dept@calpoly.edu. The specifications for the Instron 4400R are outlined below. The specifications for the Instron 1331 machine could not be obtained, and Instron no longer provides specifications for this older model; however, it does have around $100 \mathrm{kN}(22,000 \mathrm{lb})$ load cell capacity. 
Table 10: Specifications for Instron 4400R located in Building 192-135 on Cal Poly's campus.

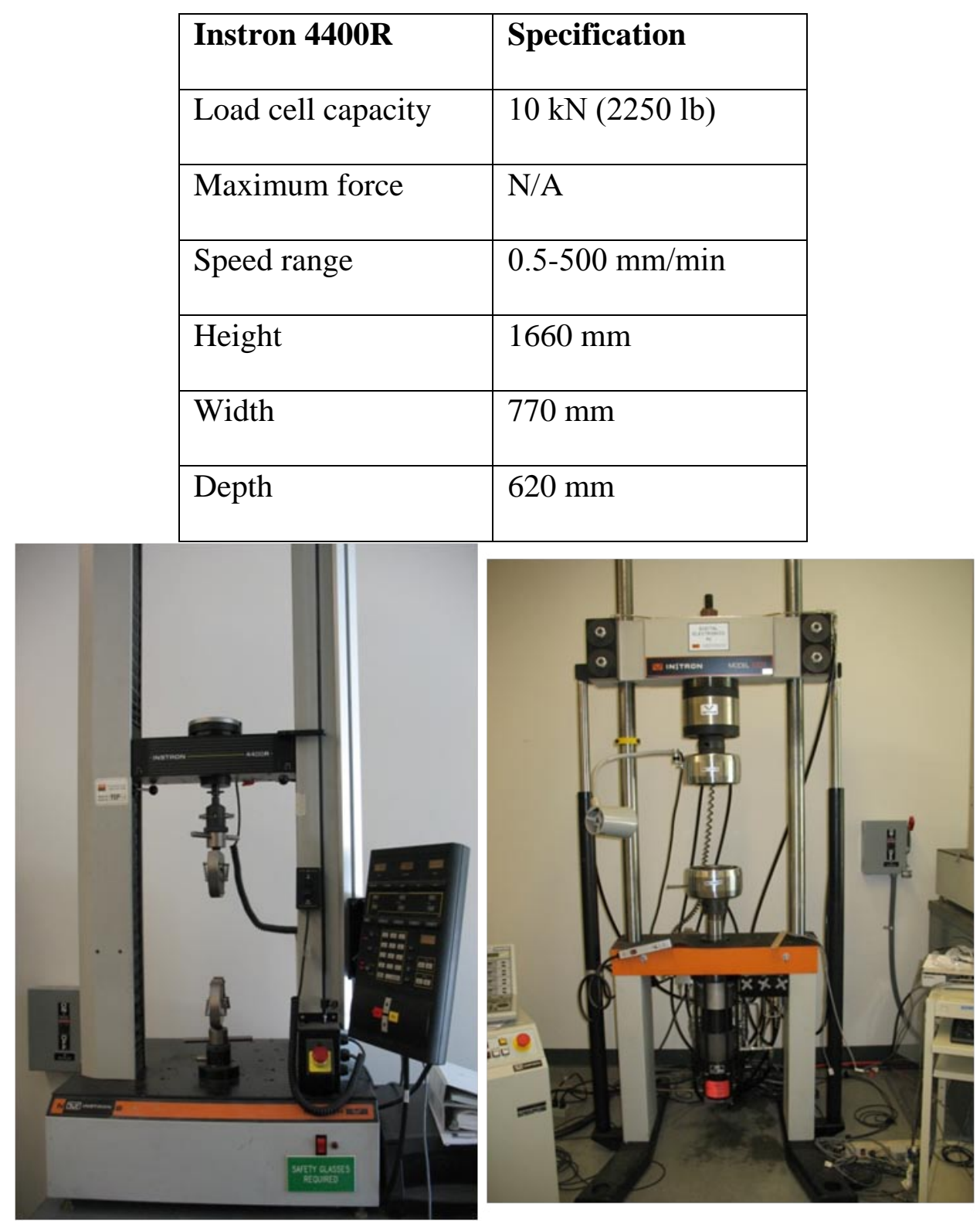

Figure 17: Mechanical Engineering department tensile testing machines. On the left, Instron model 4400R. On the right, Instron model 1331. Both machines are located in Building 192-135. 


\subsection{Summary}

There are a total of five tensile testing machines available for student use on Cal Poly’s campus that offer a range of sizes and strengths to test practically any material sample in a "dog bone" shape as described previously in Section 1.5. There is currently no fixture on Cal Poly's campus, however, that is equipped to test the tensile properties of small diameter materials in tubular form. Thus, it is the goal of this thesis to produce a test fixture that will accurately test these properties using the Biomedical Engineering department's Instron In-Spec 2200 tensile testing machine. The following chapter will outline the design requirements for the fixture that will test the transverse tensile properties of tubular materials and briefly describe each design iteration leading up to the final design prototype. 


\section{Chapter 3 - Initial Design of Transverse Test Fixture}

This chapter will outline the design requirements that needed to be met for the final design of the transverse test fixture to determine the transverse tensile properties of small diameter polymer tubes. This chapter will also describe the initial design iterations leading up to the final design. Design requirements were determined based on the Instron testing machine that the fixture would attach to as well as the size and shape of the test samples to be tested. Each requirement will be clearly stated below, followed by a discussion of the justification and importance of each criterion.

The first design requirement that needed to be met was based on the shape of the material test sample. The sample must be able to remain in tubular form while testing, as opposed to being cut into a flat test strip as described in the transverse test methods section in Chapter 1. When a tube is cut into a flat test strip and pulled in a uniaxial direction, the forces acting upon any point in the sample are in one direction. In its tubular form, however, forces acting upon any point in the sample are in two directions.

Arsene et al. describes a method of testing transverse tensile properties of tubes so as to reduce the bending moment created by the space in the split disk method [44]. This method, involving three pieces inserted inside the test sample, would be difficult to implement in the specific tubes of interest to this thesis due to the size of the inner diameter. Another design that has been successful in studies is the ring test method shown in Figure 6 in Chapter 1. Because the test sample should remain as close to its tubular shape as possible while testing, the rods used to pull the tubular test sample apart should look similar to the D-shaped blocks used in the split disk method shown in Figure 
5 in Chapter 1. This method is outlined in both ASTM D 2290 and ISO 7198. Because both ASTM and ISO are internationally recognized standards, these will be used as templates for the transverse test fixture design. While ASTM D 2290 claims to accommodate any size thermoplastic tube, the dimensions described for the notch size of the test sample are not small enough for a polymer tube with a $4 \mathrm{~mm}$ inner diameter. Thus, it was determined that the transverse test fixture needed to be designed similar to the model described in ISO 7198.

The second design requirement was based on the need to interface with the existing Instron machine. There were two main components that needed to be incorporated into the transverse test fixture design: a part to fit into the grips of the Instron tensile testing machine and a part that would apply the test load to the tube. These parts could be permanently connected or have some type of connection point; however, the connection needed to be able to withstand and accurately transfer the force applied during the tensile test to the test sample. These two components would make up one side of the test fixture with the opposite side being the mirrored design in order to pull the test sample apart in a uniaxial direction.

The grips on the Instron testing machine are two $25 \mathrm{~mm}^{2}$ blocks that clamp down on the fixture via a screw fastener shown in the picture below. 


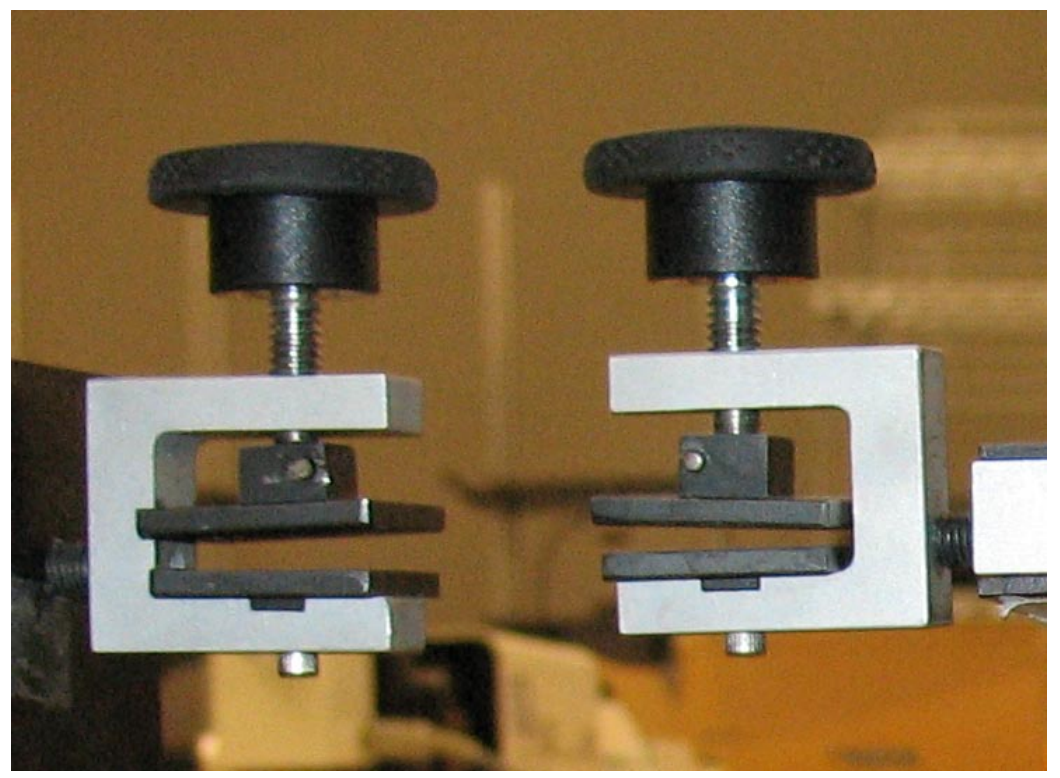

Figure 18: Instron In-Spec grips fixate test fixture to apply test load to material. Round screw fasteners above grips ensure the fixture is tightly secured.

Because these grips only widen to about $5 \mathrm{~mm}$, the thickness of the part of the fixture that fits in the grips of the Instron need to be less than $5 \mathrm{~mm}$. The width and length of this part could potentially be greater than $25 \mathrm{~mm}$, but it needed to have dimensions sufficient for it to be tightened and held sturdily in the Instron grips.

The part of the fixture that will apply the test load to the tube needed to be designed to keep the test sample in its tubular shape as much as possible due to the direction of the applied force, as described at the beginning of this chapter. In order to do this, the two hooks or rods used to pull apart the test sample needed to conform to the tube's original shape. This required a rounded rod with a similar radius to the tubes being tested. Because both the PLGA and ePTFE tubes being tested on Cal Poly's campus have approximately $4 \mathrm{~mm}$ inner diameters, the rod had to have around a $2 \mathrm{~mm}$ radius. Furthermore, the two rods that will be pulling the sample apart must initially be 
able to fit into the test sample before testing. This means that the two rods combined must fit into a $4 \mathrm{~mm}$ diameter circle.

The final design requirement concerned the fixture material itself. The material chosen for the test fixture must withstand the load needed to pull apart the test sample. Therefore, the test fixture material must have stronger tensile properties than the tubular materials being tested (i.e. PLGA and ePTFE). The overall objective of this design is to accurately test the transverse tensile properties of small diameter tubular polymers. Therefore, based on the criteria outlined and discussed above, the final transverse test fixture must include:

- A component that will fit into the Instron machine grips and accurately transfer the test load to the tubular test sample; therefore, it shall be no thicker than $5 \mathrm{~mm}$, and not larger than $25 \mathrm{~mm}$ in width and length.

- A component that will fit into the tested tubular material and apply the test load to the test sample. This component shall be no larger than $1.9 \mathrm{~mm}$ in radius in order to give a tolerance to the $2 \mathrm{~mm}$ inner radius of the tubes to be tested.

- Two rods that will pull the material in a uniaxial direction while keeping the test sample as close to its original tubular form as possible

- A fixture material that is strong enough to withstand force needed to pull apart the tubular material being tested. This force shall not exceed $50 \mathrm{~N}$, based on initial pilot tests. 


\subsection{Initial Design Models and Concepts}

In order to create a test fixture that satisfied the established criteria, initial designs were brainstormed and drawn out in a laboratory notebook. These initial designs did not take into consideration manufacturability or ease-of-use for the person performing the test. The focus was exclusively on creating a fixture that would fit onto a tubular test sample and accurately apply the load of the Instron testing machine. After a general sketch identified the necessary components for any design, each specific design iteration was modeled using SolidWorks 3D modeling software. The original idea for the test fixture was modeled after the transverse test fixture described in ISO 7198. A drawing of this general sketch is shown below in Figure 19. The connection grip shows where the fixture would fit into the Instron grips. The two rods, when placed next to each other, would slide into the test sample. Subsequently, the load from the Instron machine would pull the two rods apart until the tubular sample breaks. 


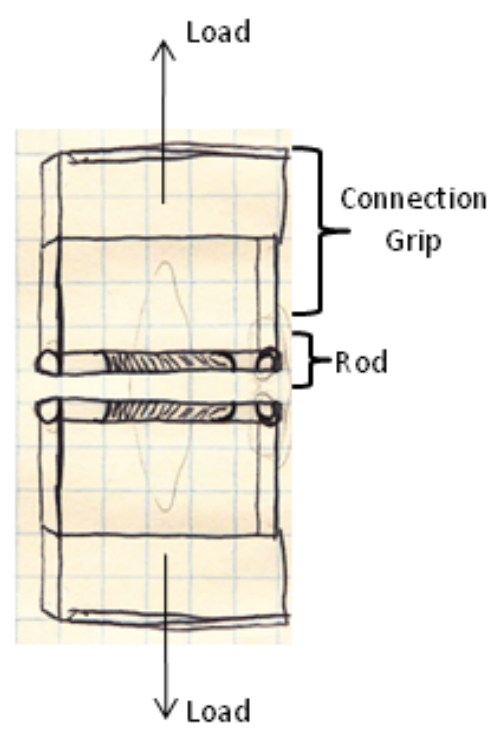

Figure 19: Initial sketch of transverse test fixture. The connection grips show where the fixture would be secured in the Instron machine's grips. The rod is what would support and apply the load to the tubular test sample. The shaded area on the rod shows where a tubular test sample would be placed.

The dimensions of the cross section of the rod needed to fit closely with the shape of the $4 \mathrm{~mm}$ inner diameter of the test samples; thus, the rod was designed with a $0.1 \mathrm{~mm}$ tolerance so the test sample could easily fit onto the test fixture. A closer look at the cross section of the rod is shown below. 


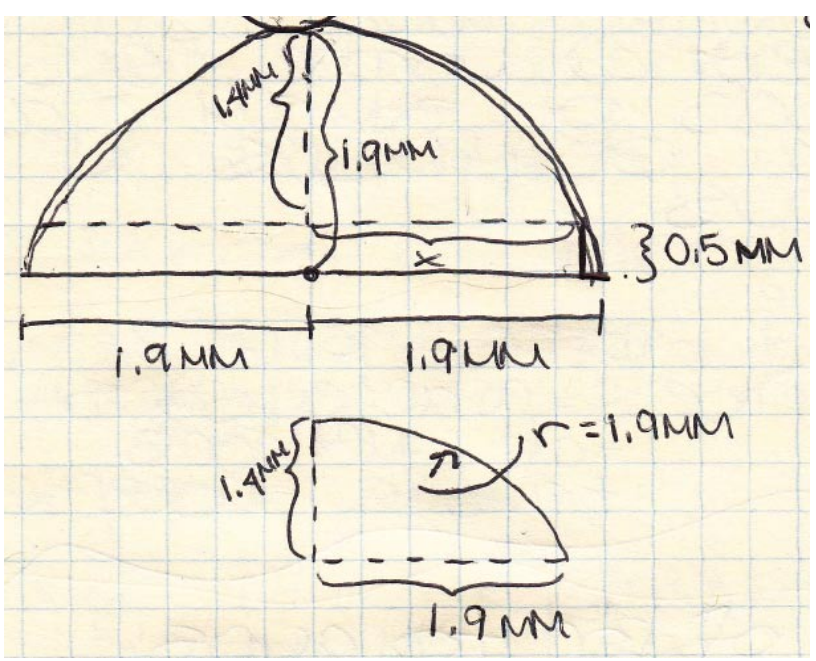

Figure 20: The top semicircular shape shows a cross-section of the initial rod design and dimensions. The height is $1.4 \mathrm{~mm}$ in order to provide a $1 \mathrm{~mm}$ tolerance between opposing rods during testing. The bottom sketch shows half of the cross-sectional area of the rod.

The next consideration in the design process was how to load the test sample onto the fixture along with how the rod would connect to the connection grip. A simple screw connection seemed like a viable choice, and was incorporated into the design for the first SolidWorks iteration.

\subsubsection{Initial Design 1}

The first design incorporated the ideas from the sketch above, along with a pinhole connection to attach the rod to the connection grip. The rod of this design was made with an inset for the test sample to be placed. This assures that the sample would not slide along the rod during the test. In order to accurately transfer the load from the connection grip to the rod without any bending, the grip was designed to support the rod on both sides of the test sample. This caused an extra $0.5 \mathrm{~mm}$ of material below the rod 
on the connection grip. Because the two rods would need to fit inside the tubular sample with a $2 \mathrm{~mm}$ inner radius, the height of each rod can be no longer than $1.5 \mathrm{~mm}$ to allow for the $0.5 \mathrm{~mm}$ gap caused by each connection grip. A hole was placed on the exterior of the design to allow for a screw or a pin to hold the rod and connection grip together. This hole was placed outside the main body of the connection grip so that the screw or pin would not interfere with the grips of the Instron testing machine. A drawing of this initial concept is shown below.

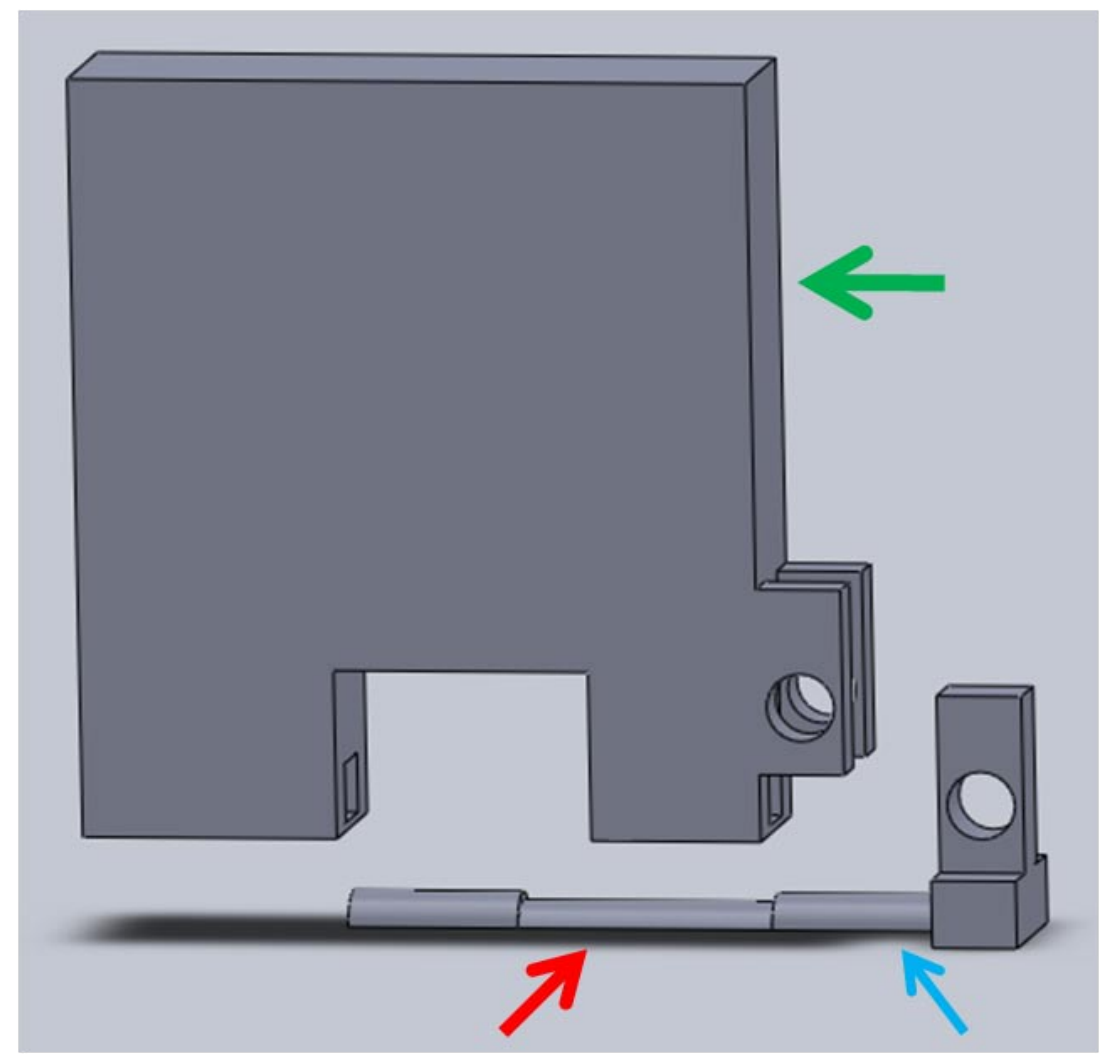

Figure 21: SolidWorks model of initial design 1. Green arrow points to connection grip. Blue arrow shows rod and connecting pinhole to connection grip. Red arrow points to inset on rod to ensure test sample does not slide along rod. 
After a SolidWorks model was made, the part was rapid prototyped using a fuse deposition modeling machine located on Cal Poly's campus. A piece of ePTFE and PLGA were placed on the rods and connected to the connection grip to see how a test sample would fit onto the fixture. Below shows a picture of the connection grips for initial design iteration 1 . The rapid prototyped rods had broken by the time the picture was taken.

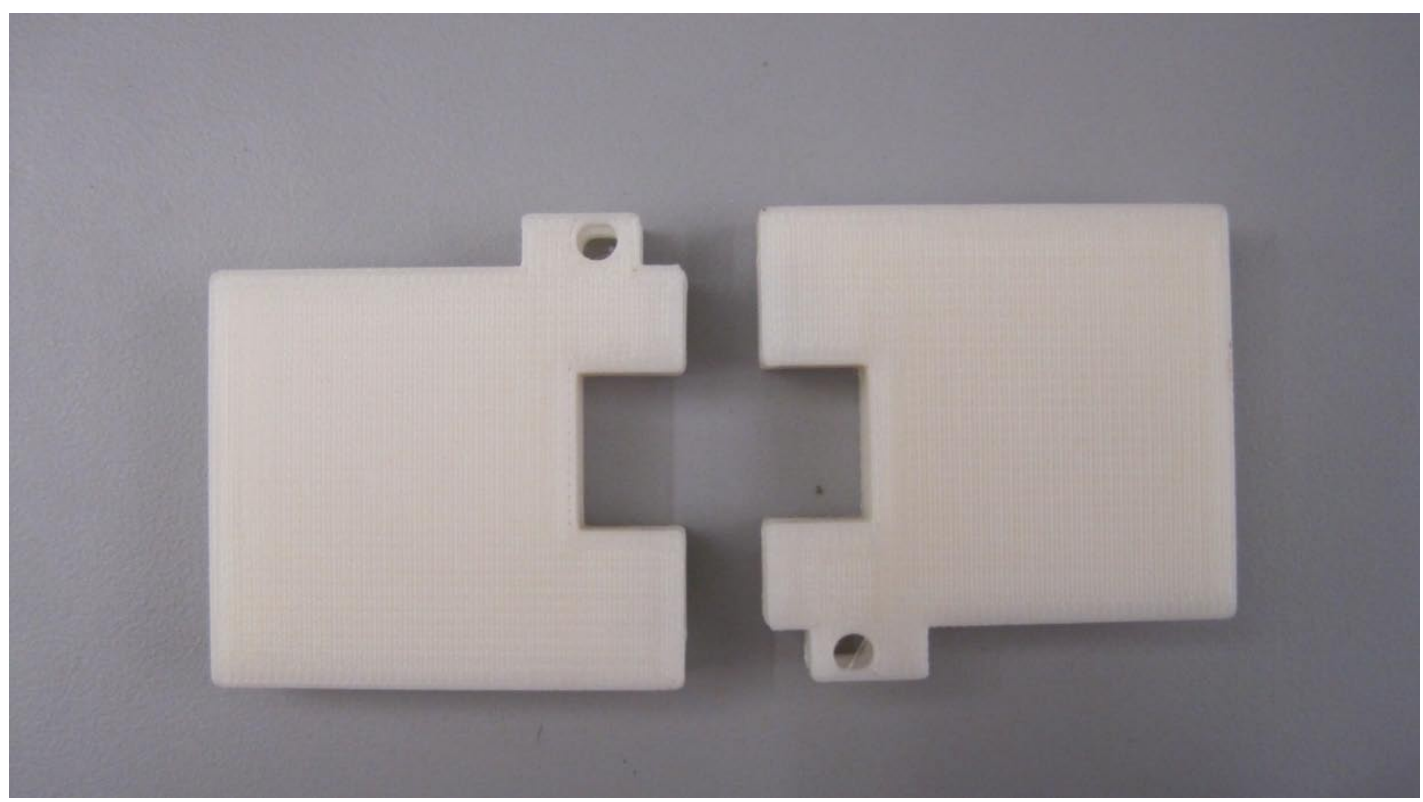

Figure 22: Connection grips of initial design iteration 1. The two grips are shown as they would be aligned in the Instron testing machine.

In evaluating the rapid prototyped model, the most important aspect was the fit of the test sample onto the two rods. The rods kept a radius of $1.9 \mathrm{~mm}$ to fit snugly into the $2 \mathrm{~mm}$ radius of the tubular test samples; however, the height of the rod was designed to be $1.4 \mathrm{~mm}$ to account for the $0.5 \mathrm{~mm}$ gap caused by the support in the connection grip previously shown in the sketch drawing in Figure 20. This caused less surface area of the inside of the tubular test sample to be covered, which is not ideal when trying to keep the 
test sample as close to tubular form as possible during testing. The samples did not fit snugly when connected to both connection grips due to the large $1 \mathrm{~mm}$ gap in between the two rods. However, in spite of this limitation, the pinhole connecting the rod and the connection grip seemed to accurately transfer a load to the test material, and the inset kept the material from sliding along the length of the rod. Because of the strength and texture of the rapid prototype material, however, these criteria could not be verified until a stronger model of the fixture was made.

Therefore, the most important feature to improve was creating a rod that could sit flush against the opposing rod to fully encompass the inner radius of the tubular sample. Another design was needed to improve upon this initial iteration.

\subsubsection{Initial Design 2}

The focus of the second iteration of the transverse test fixture design was to provide a surface on the rod that maximized the inner surface area of the tubular test sample, while keeping its tubular shape. Because the support provided by the connection grip was causing an ill fit of the test sample on the two rods, this second iteration used two different rod designs to avoid the gaps caused by the connection grip. The connection grip design remained similar to that described in the first initial design. The only significant change made to the second iteration was the removal of the support for the rod on the side closest to the pinhole. This was removed to allow for the new design of the rod. The second initial design of the rod actually used two different rod designs. The first, shown below on the right, was similar to the first iteration of the design. The second rod, shown below on the left, dropped below the bottom plane of the connection 
grip, which provided a flush interface with the opposite rod. This created a more circular shaped surface for the tubular test sample compared to the first iteration of the design.

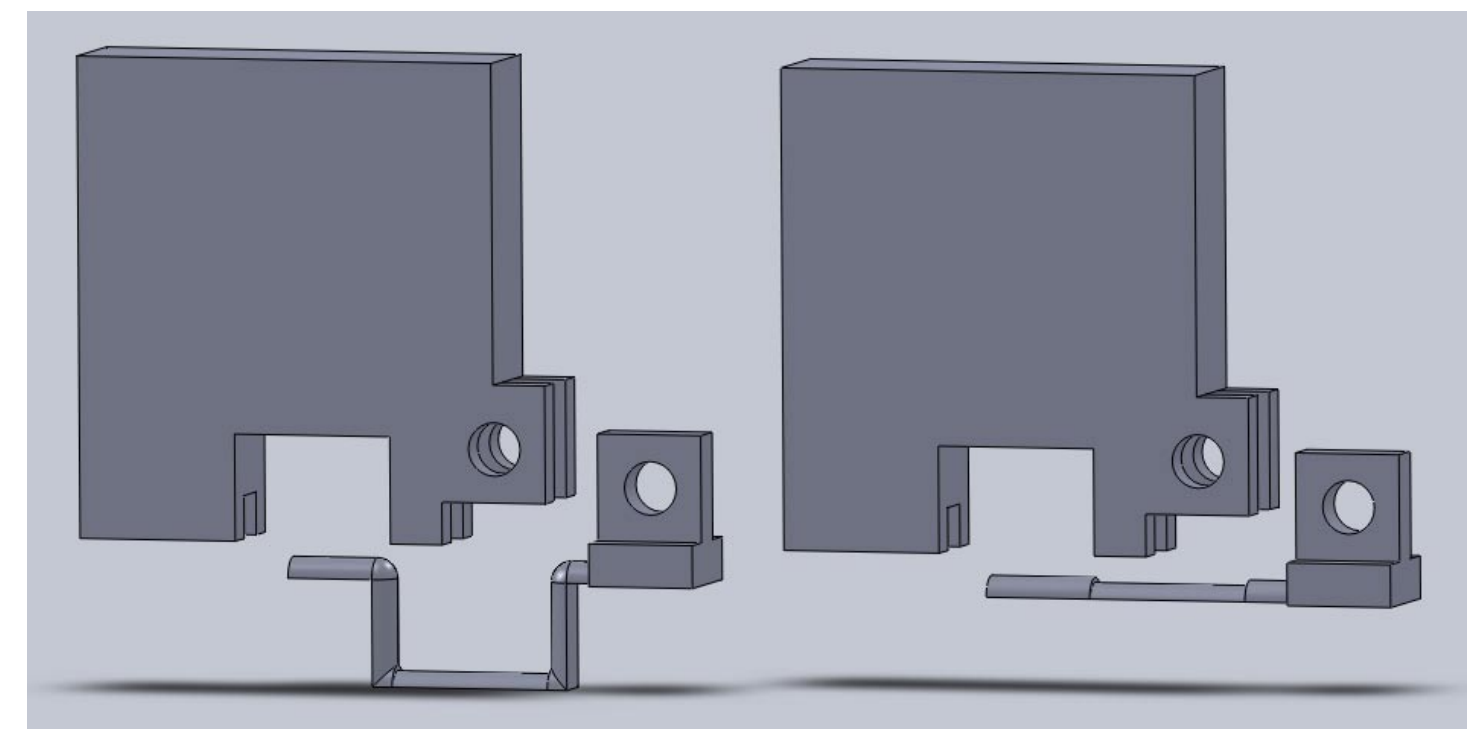

Figure 23: Solidworks model of initial design 2. Two different rods were designed so they would be flush against each other, making sure the tubular test sample was kept in its original shape.

Figure 24 below shows the rapid prototyped part of the second design iteration's SolidWorks model, using the same fuse deposition modeling machine mentioned earlier. The part looked promising, and the ePTFE fit well onto the two rods without any gaps caused by the connection grips. When PLGA was fit onto the fixture, however, it was extremely difficult to slide the test sample around the 90 degree corner of the rod, circled in red in the figure below. 


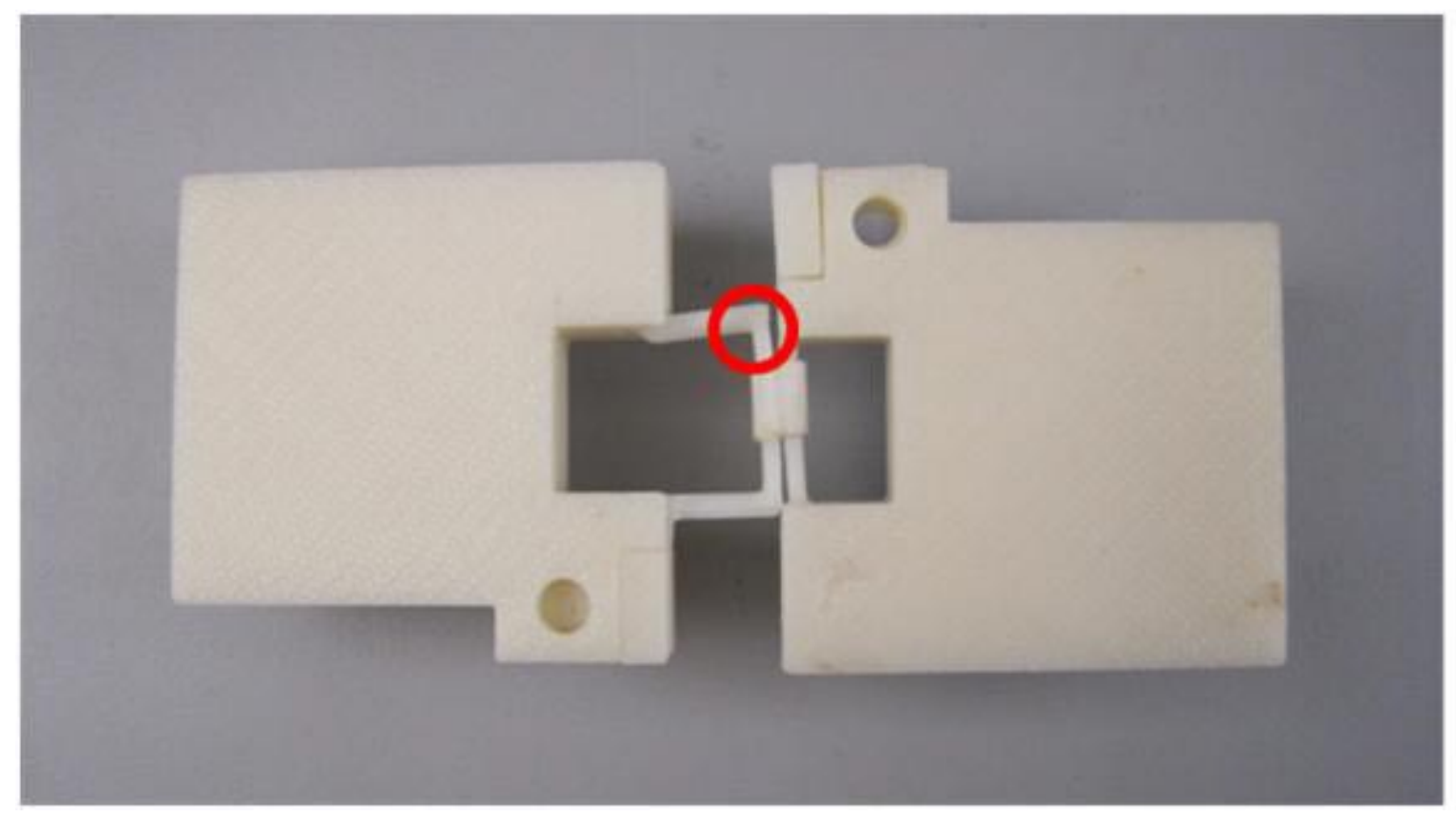

Figure 24: Rapid prototyped model of intial design 2 with sample ePTFE inserted in rods. The 90 degree angle circled in red shows where the tubular PLGA samples

\section{had a difficult time sliding around.}

PLGA is stiffer than ePTFE, and this design did not cater to the material's lack of flexibility. While this design may have been ideal for ePTFE, it would not have worked for testing samples of PLGA or other stiff materials. This design would also have been slightly more tedious to manufacture due to two different rod designs. It would be ideal to have a fixture that was identical for both sides of the test piece for ease of manufacturing. This would also be helpful in case one side of the fixture broke it could be easily replaced using one universal design drawing for the fixture. Therefore, although this design allowed the two rods to be flush against each other thus allowing the material to stay in its original shape, improvements needed to be made in order to allow for testing of more stiffer materials, such as PLGA, that were difficult to slide onto the rod with a 90 degree angle. 


\subsubsection{Initial Design 3}

The third iteration for the test fixture was designed to satisfy the needs identified from the first two iterations, and additionally take into consideration ease of manufacturability. Thus, the rod was redesigned so that the same part could be used on both sides of the fixture. Another requirement, realized after Initial Design 2, was that the rod must be strictly horizontal so that stiffer materials, such as PLGA, could easily slide onto the rod. The third iteration incorporated both of these ideas by having the rod bend down from the pinhole, as shown in the drawing below, then fit into a support in the connection grip on the opposite side.

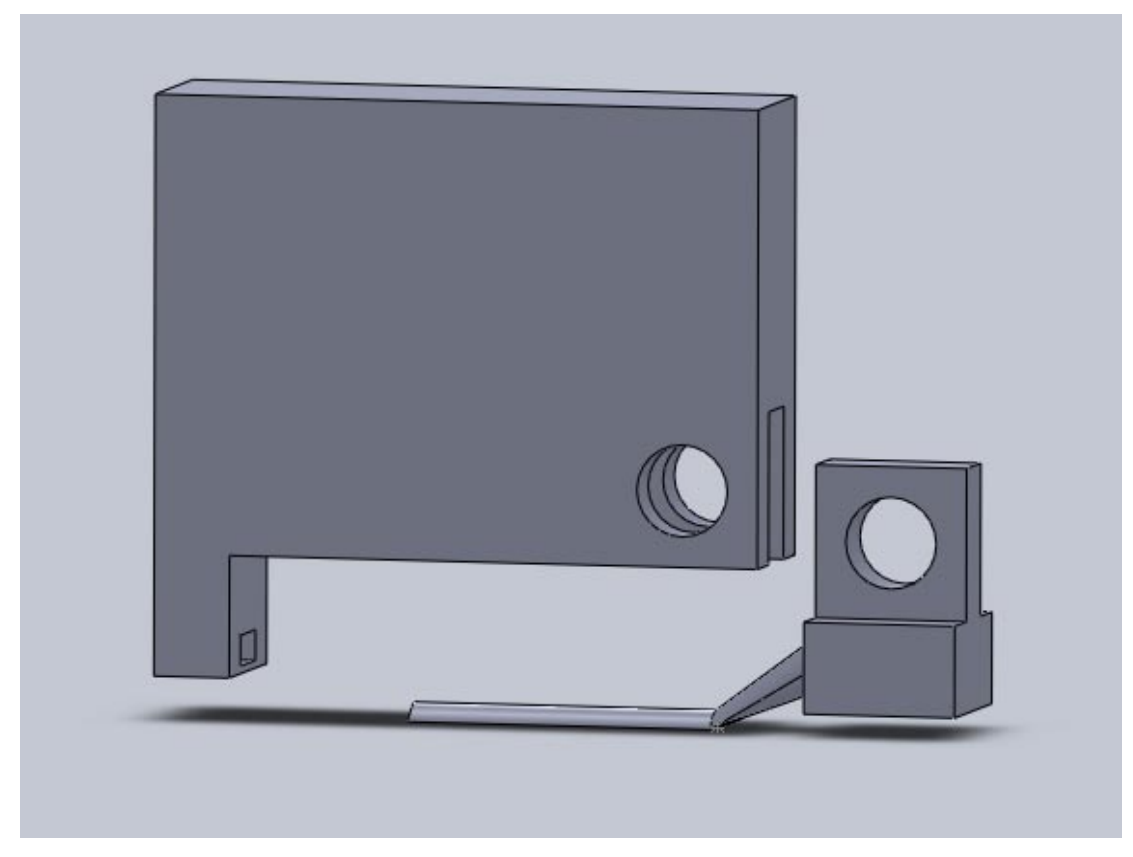

Figure 25: Solidworks model of initial design 3.

By situating the two parts of the fixture opposite to each other during testing, as in the pinholes of the two parts located on opposite sides, the test sample will still sit flush with the rods. This is depicted in the picture below of the rapid prototyped model of this 
design iteration. This design also allows the material to be easily slid onto each rod. The inset originally designed on the rod was removed on this iteration of the design because it was realized that the force applied to the material would only be perpendicular to the rod, thus preventing any sliding of the material along the test fixture during a tensile test.

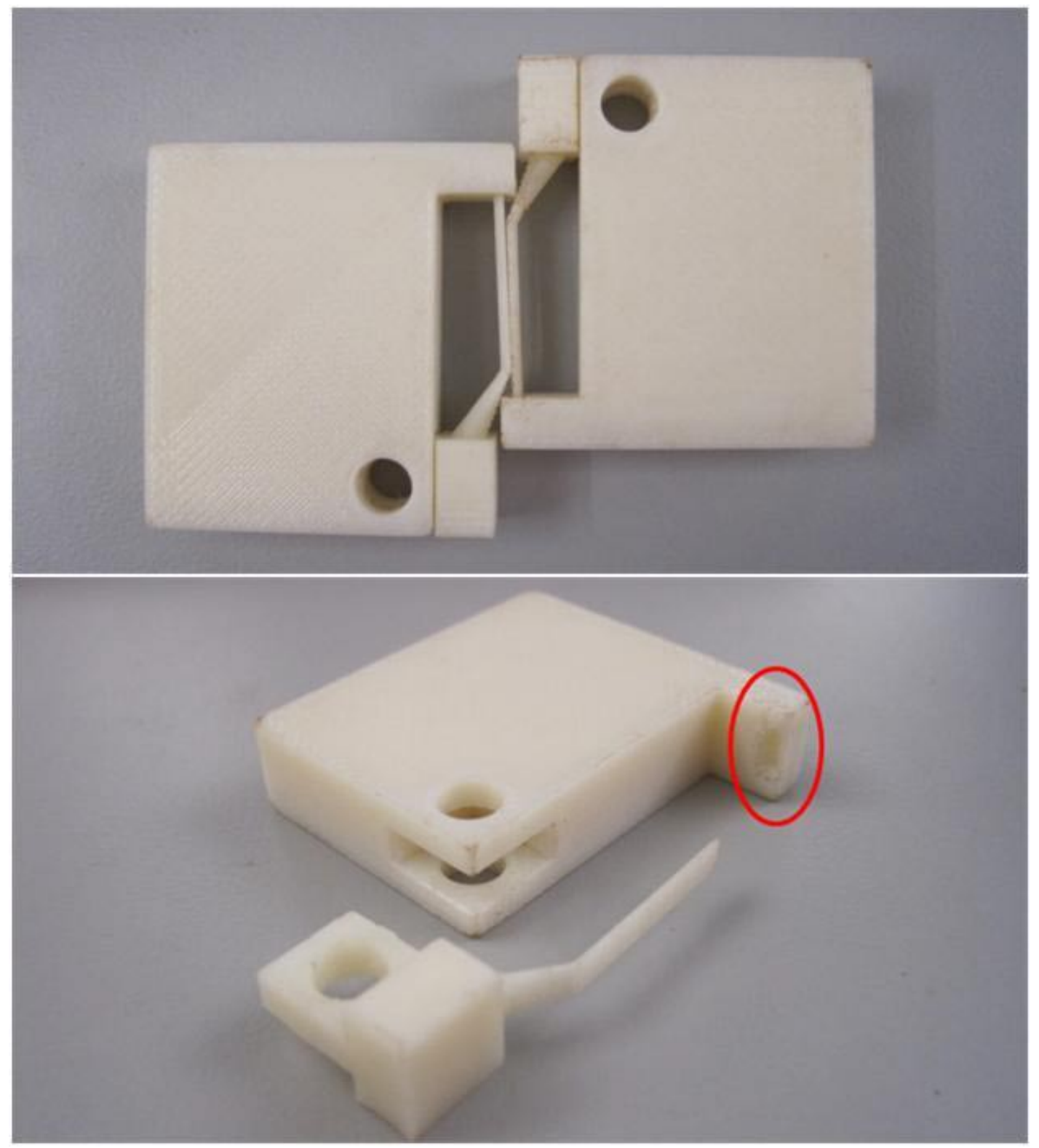

Figure 26: Rapid prototyped model of initial design 3. The top picture shows how bending both rods provided a flush surface against each other, while eliminating the 


\section{difficult angle for stiffer materials to slide around. The bottom picture shows the slot, circled in red, that supports each rod.}

After evaluating the rapid prototyped model of this design iteration, it seemed as though this was a promising model for the final design of the transverse test fixture. After seeing how the rapid prototyped model would fit in the Instron machine, the only modification to be made during manufacturing was to decrease the thickness of the connection grip to $4 \mathrm{~mm}$ (originally $10 \mathrm{~mm}$ ) in order to fit in the grips of the Instron machine. The part was taken to the Mustang '60 machine shop on Cal Poly's campus, and the connection grip was manufactured first using a computed numerically controlled (CNC) machine. The material used was 3/8” mild steel beam purchased from McCarthy Steel in San Luis Obispo, CA. The figure below shows the manufactured connection grip.

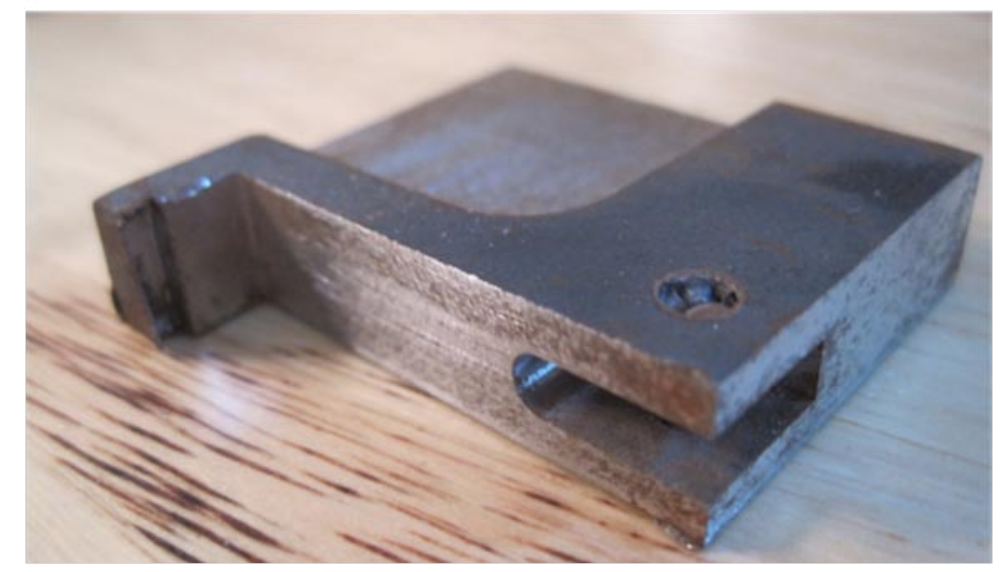

Figure 27: Manufactured connection grip for initial design 3. Part was made out of 3/8" mild steel beam in Cal Poly's Mustang '60 machine shop.

The slot in which the rod was supported by the connection grip turned out to be harder to manufacture than originally thought due to the small dimensions of the part. 
With a thickness of $0.5 \mathrm{~mm}$, the end of the slot was angled outward due to the larger size of the end mill in the CNC machine. A smaller end mill could not be used as it would not be strong enough to cut the steel beam. A close up of the slot is shown below, which would clearly not be useful as a support to the rod.

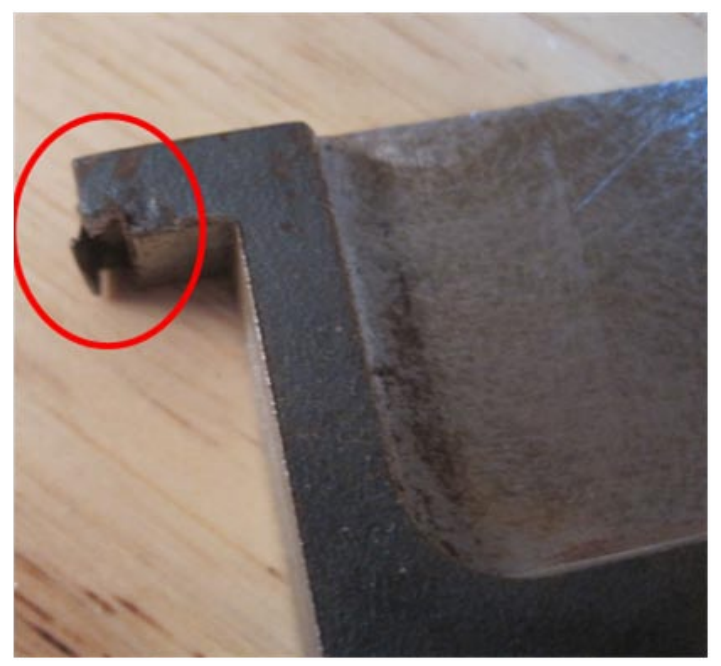

Figure 28: Manufactured connection grip of initial design 3. Red circle shows where the slot is angled outward, making it unsuitable to support a rod.

Another manufacturing issue was addressed with regards to how the rod was going to be attached to the pinhole. Originally the plan was to press fit a steel rod into a machined part where the pinhole would be. Subsequently, the rod would be ground down to the correct height and bent to form the drop down model previously shown in the SolidWorks drawing in Figure 25. After consulting with machine shop technicians, this did not seem feasible. It was then back to the drawing board to find a solution to these manufacturing problems. 


\subsubsection{Initial Design 4}

When considering manufacturing for the third design iteration, steel was chosen for the material of the test fixture. After running into issues with the manufacturability of the slot to support the rod, it was discussed whether or not a support for the rod was even needed. Under the assumption that steel would be strong enough to withstand the ultimate tensile strength of the material, the support from the connection grip was removed from the fourth design iteration. Because the rod no longer used support from the connection grip, the material could easily slide onto the end of the rod, eliminating the need for a pinhole connection between the rod and connection grip. Thus, a SolidWorks model of the fourth, and final, design iteration is shown below.

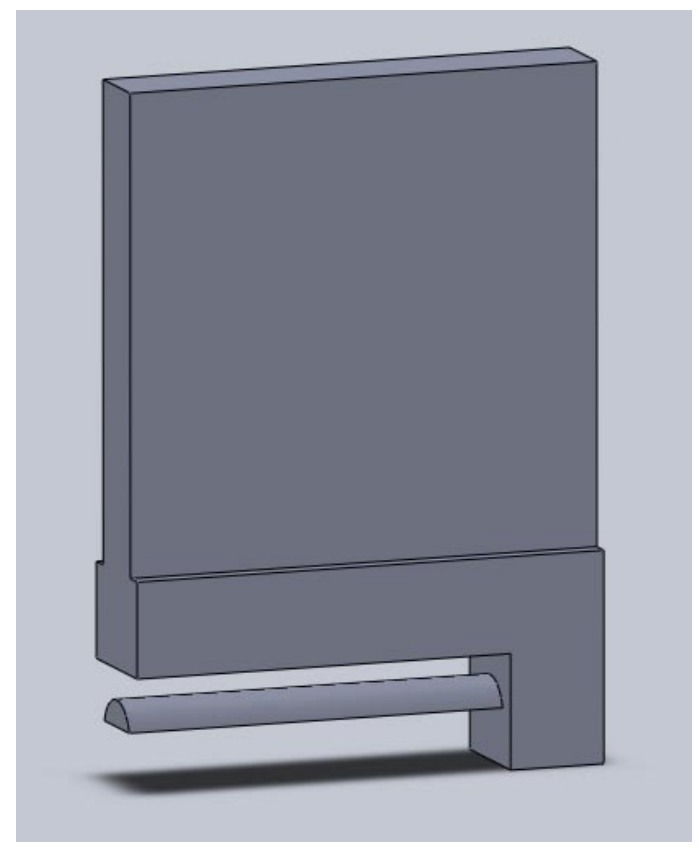

Figure 29: Solid works model of design iteration 4, chosen for the final design.

A rapid prototyped model of the fourth design iteration was made and evaluated using test samples of ePTFE and PLGA. Both materials fit well onto the rods, as shown 
below, with the two parts placed opposite of each other to prevent the connection grips from running into each other.

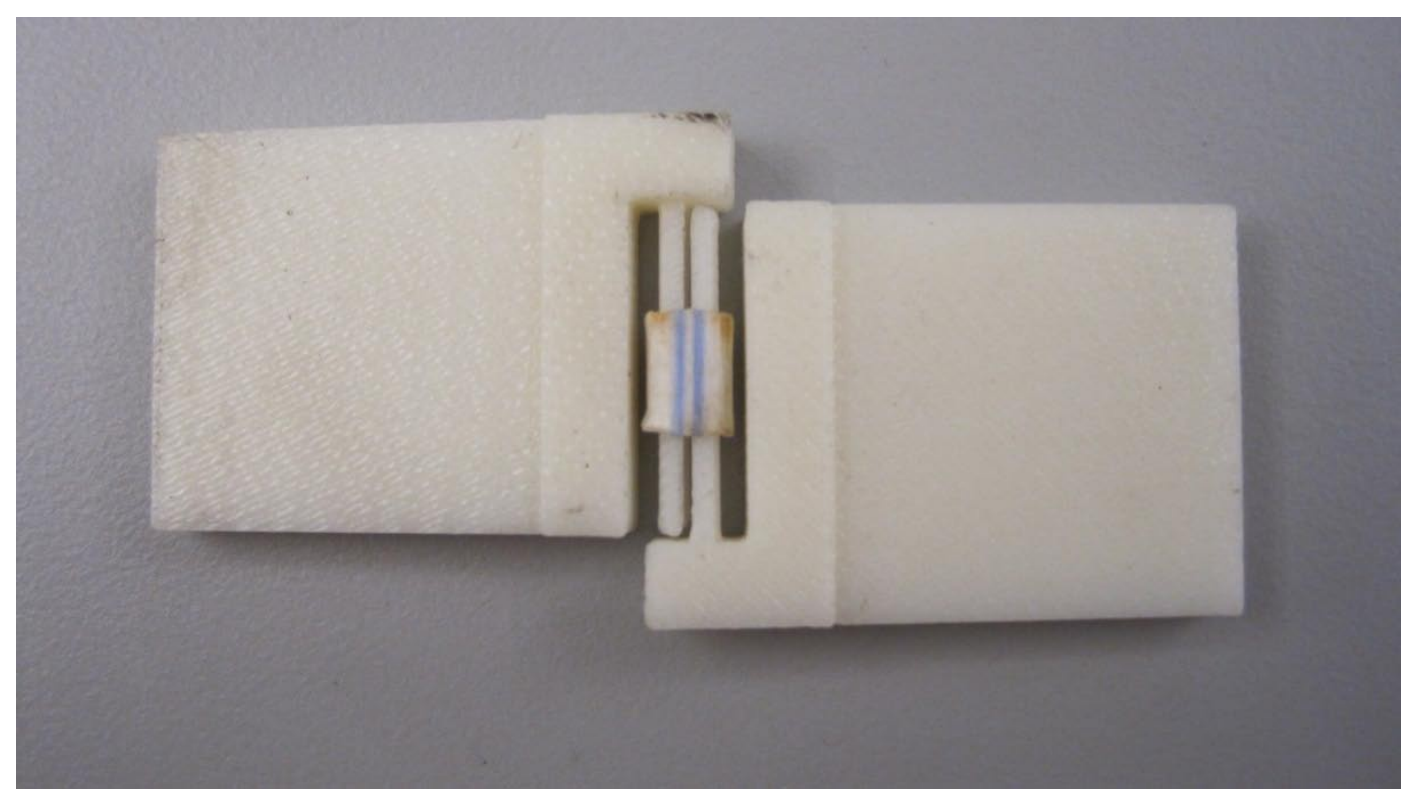

Figure 30: Rapid prototype model of final design with sampleof ePTFE attached.

The part was then tried out in the Instron machine grips to assure fit. The design worked well with the test material as well as with the Instron machine, as shown below. 


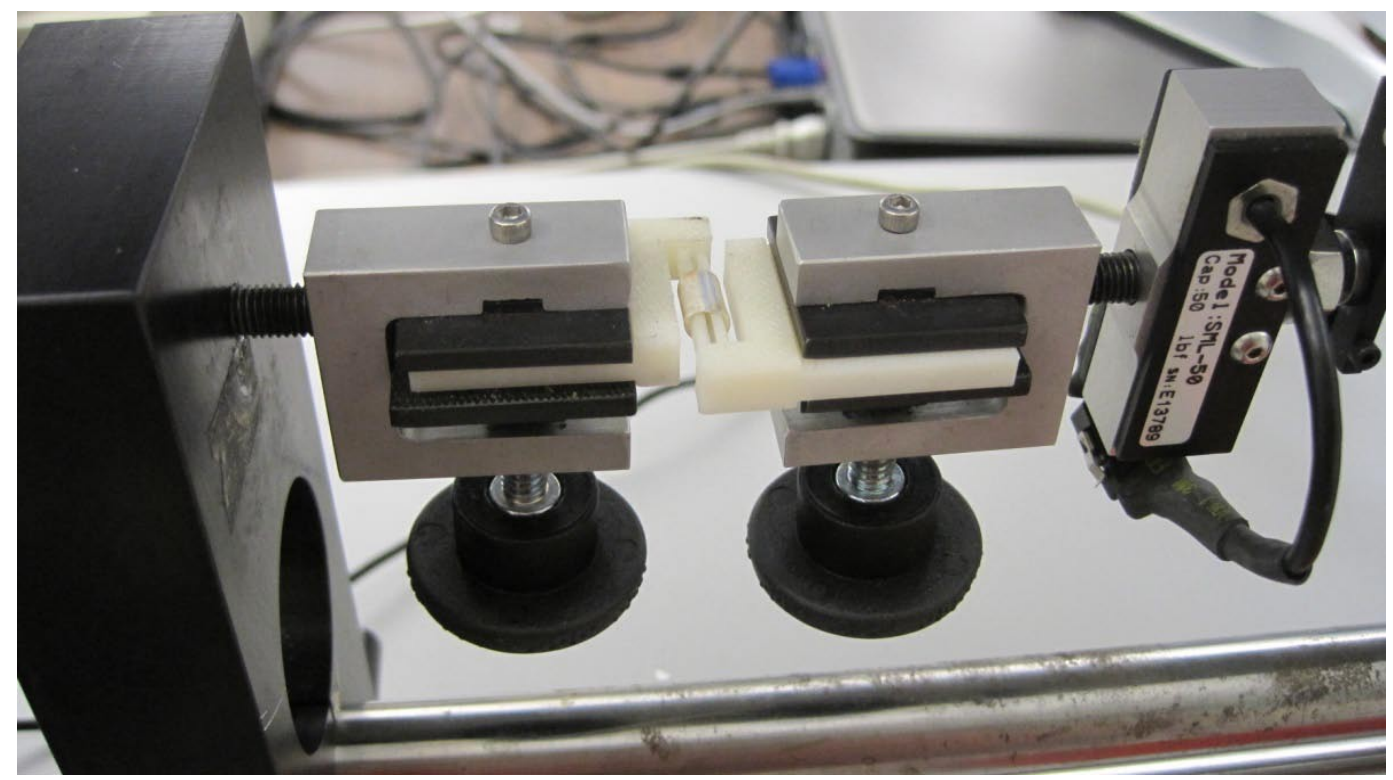

Figure 31: Final design rapid prototype being tested in Instron grips to assure fit. Sample of ePTFE attached to prototyped fixture.

The manufacturability was considered in the creation of this design iteration and, after consulting a machine shop technician and analyzing the rapid prototyped model, this design was deemed feasible to make using mild steel. Thus, the fourth design iteration was chosen to move forward with as the final design. The following chapter will describe the details of the design and the manufacturing process used. 


\section{Chapter 4 - Final Design of Transverse Test Fixture}

\subsection{Introduction}

As mentioned in Chapter 1, it is important for a vascular graft material to have similar tensile properties to the native vessel that the graft is replacing [4]. In order to accurately test the transverse tensile properties of vascular graft materials, the material must remain in tubular form during testing. Thus, it is the goal of this thesis to create a test fixture that will more accurately test the tensile properties of both ePTFE and PLGA tubes compared to the current method of testing. The current method consists of cutting the tube and laying it flat, then pulling on both sides in the transverse direction.

Chapter 3 outlined the design criteria required for the transverse test fixture design, and documented the design iterations and prototypes throughout the design process. The most important of these requirements was the need for the final design to keep the test material in its tubular form. Both the ePTFE and PLGA tubes that were to be tested using this fixture have a $4 \mathrm{~mm}$ inner diameter; thus, the final design must incorporate a rod that fits these dimensions in order to apply the test load to the tube. Furthermore, the fixture must be able to fit into the $25 \mathrm{~mm}^{2}$ grips of the Instron machine with a thickness of $5 \mathrm{~mm}$.

The fourth and final iteration was chosen for the final design because the prototype met all design requirements. Additionally, after consultation with Dave Laiho, a technician for the BMED department, this design was deemed easiest to manufacture compared to the other design iterations. The final design prototype was tested for fit in 
the Instron grips, and seemed easy to use when mounting the material onto the rods and securing the fixture in the Instron grips. This chapter will describe in detail the final design of the transverse test fixture including how it was constructed, how it was tested, as well as how it shall be used.

\subsection{Materials and Methods}

\subsubsection{Transverse Test Fixture}

The connection grip for the transverse test fixture was made out of the same mild steel beam (3/8") used in the third design iteration. The SolidWorks drawings were then taken to Mustang '60 Machine Shop on Cal Poly's campus to be made using a CNC machine. CNC machines are automated tool machines that use a computer language called G-code to program the machine to construct a part. The part is constructed by cutting away pieces of material from a solid block. Material can range from soft foam or plastic to hard metals [55].

In order to create the half cylinder rod that would support the test material and apply the test load to the sample, it was decided that a cylindrical rod should be press fit into the connection grip then ground down to make a half cylinder. A 0.1485 ” diameter hole was drilled into the face where the rod was to be placed in order to press fit a steel rod into the connection grip. Tolerances for a typical press fit are 0.001 ”; thus, 0.1485 ” hole was drilled to accommodate a 0.1495 ” diameter rod [56]. 


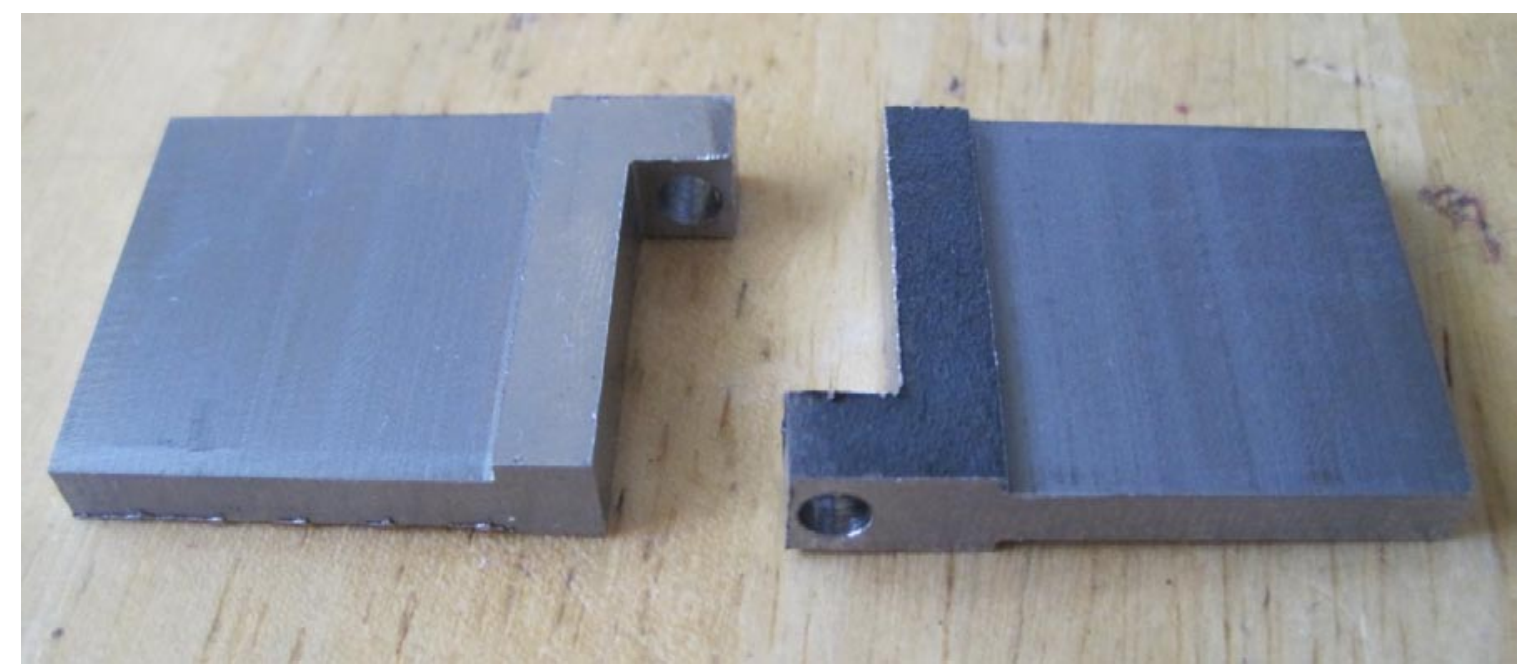

Figure 32: CNC machined connection grip. 0.1485 ” holes were drilled in order to press fit a $0.1495 ”$ rod.

In order to apply an accurate force to the test sample from both Instron grips in a uniaxial direction, the rods must be perpendicular from the face they are protruding from. Because of this, it was decided that a local machine shop would be hired to ensure the rods be perpendicular to the connection grip face. Two M2/M7 high-speed steel rods, 0.1495 " in diameter and 3" in length, were purchased from McMaster-Carr. The rods and connection grips were then taken to Precision Machine Shop in San Luis Obispo, CA. The rods were cut to $25 \mathrm{~mm}$ length, and then ground down from the middle of the cross-section to create a half cylinder shape before being press fit into the connection grips as shown in the diagram below. 


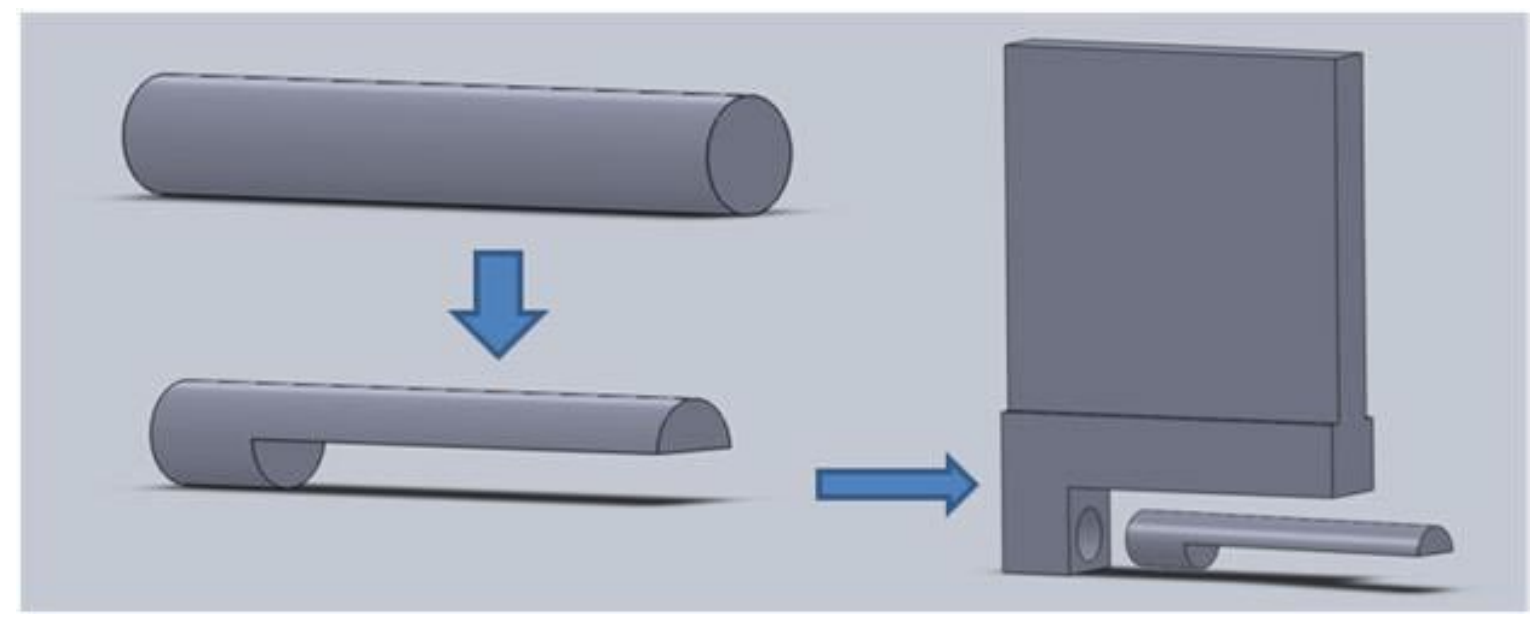

Figure 33: Two rods were cut to $25 \mathrm{~mm}$ in length then ground down $0.075 " 20 \mathrm{~mm}$ down the rod. Then each rod was press fit into the hole of each connection grip.

The final metal fixture is shown below. To prevent any moving of the press fit rod in the connection grip, a tack weld was placed on the back of the press fit also shown below. A detailed list of parts and drawings for the final transverse test fixture can be found in Appendix C.

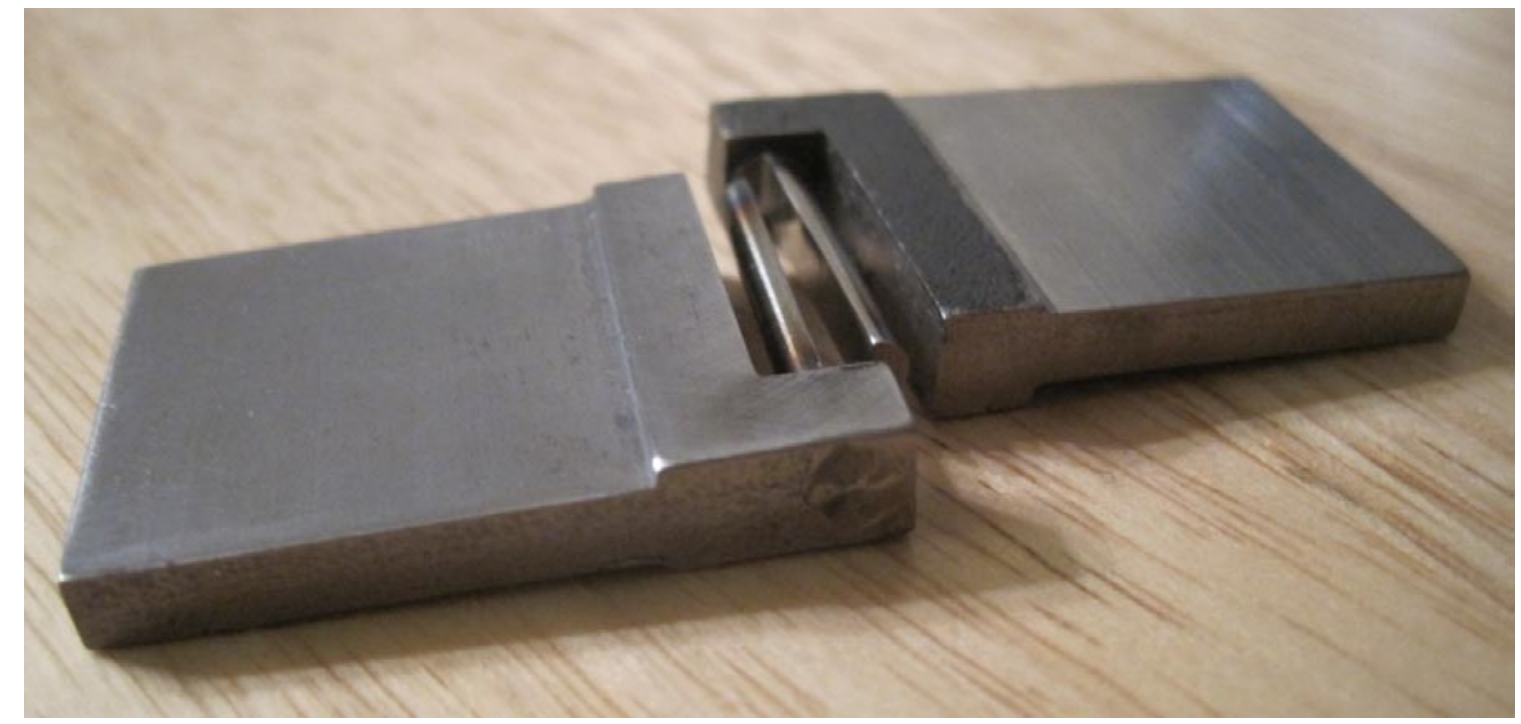

Figure 34: Final manufactured transverse test fixture. 


\subsubsection{Test Methods}

In order to test the functionality and accuracy of the final transverse test fixture, samples of ePTFE and PLGA tubing were tested for elastic modulus and ultimate tensile strength in the transverse direction using the transverse test fixture compared to the current method of testing. While this section will provide an overview of the methods used to test the final transverse test fixture design, a detailed protocol for using the transverse test fixture can be found in Appendix D and protocols for testing material using current methods and be found in Appendix E.

For the current method of testing, samples of ePTFE and PLGA tubing are cut into 5-10 mm length sections. The tubular sample would then be cut down the length of the tube flattened and inserted into the Instron grips as shown in the figure below.

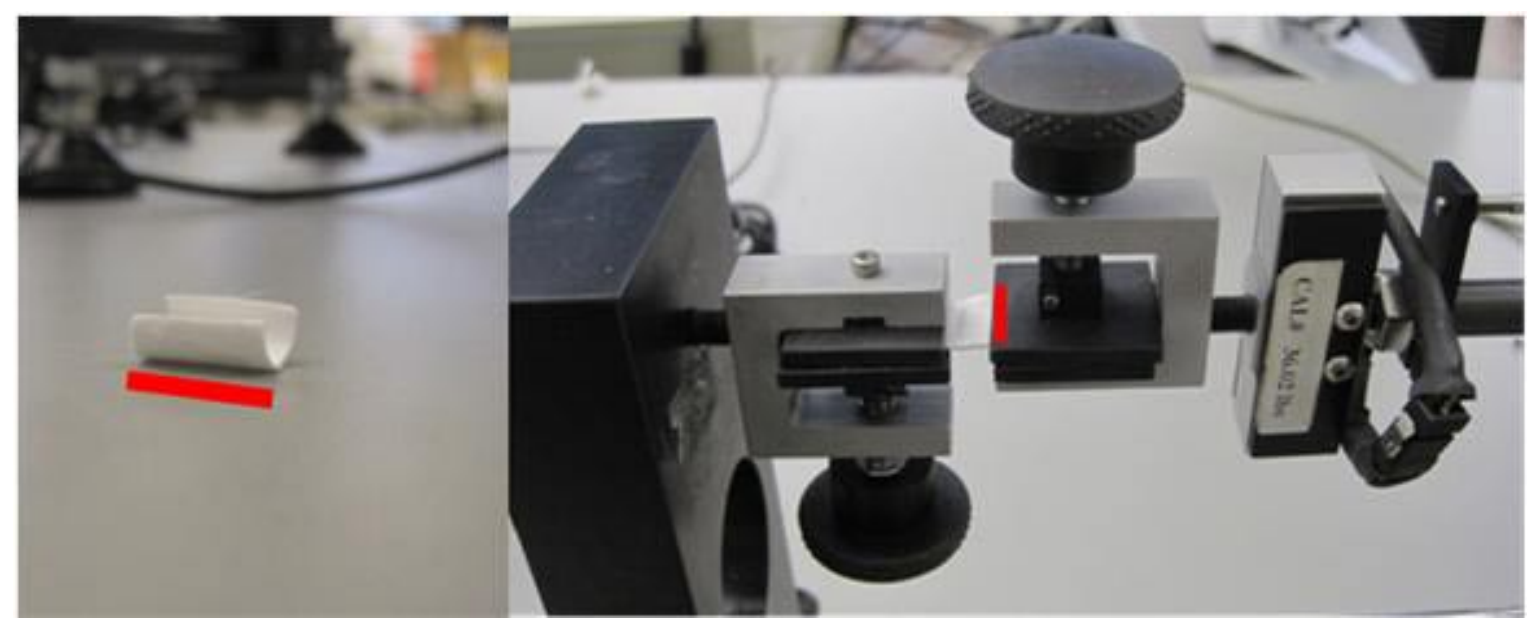

Figure 35: The figure on the left shows a test sample prepared to be tested using the current method of cutting the tube then lying it flat to be tested. The red line shows the width of the test sample, and the figure on the right shows how the sample would be aligned in the Instron grips. 
For each test, the width and thickness of the sample was measured as well as the gage length of the sample once placed in the Instron grips. All measurements were gathered using a caliper with an accuracy of $+/-0.001$ ”. The width and thickness were multiplied together to calculate the cross-sectional area of the test sample, and then divided over the load data gathered by the Instron machine to get the stress of the test sample. The strain was calculated by dividing the extension data gathered by the Instron machine by the gage length of the test sample. A stress-strain curve for each sample was then generated and subsequently, the elastic modulus and ultimate tensile strength were determined.

Material samples for testing the new transverse test fixture were prepared differently compared to the current method of testing. ePTFE and PLGA tubes were still cut into 5-10 mm length sections, but kept in tubular form. In order to localize the deformation in the test sample and pre-set gage length was cut out of the tubular samples using a 1/4” diameter hollow pin and a drill press as shown below. 


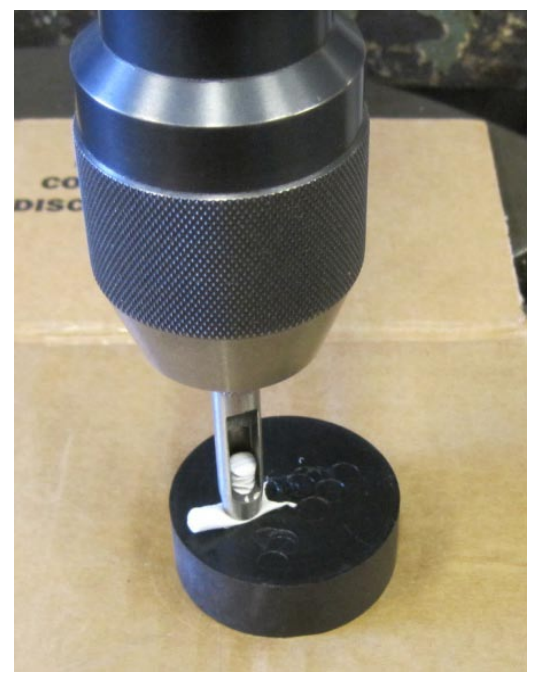

Figure 36: Drill press used to prepare notched samples of ePTFE and PLGA in tubular form.

A 1/8” radius curve was chosen due to the specifications in ASTM standard D 2290. This standard specifies that tubular test samples should have a reduced section with a radius of 1/8” [51]. After the tube was cut to the correct dimensions with a reduced section, its thickness was measured along with the width of the narrowest region of the reduced section. The test sample was then loaded onto the transverse test fixture and the fixture was secured in the Instron grips. The gage length was measured as the distance between the two rods supporting the test sample, as shown below. 


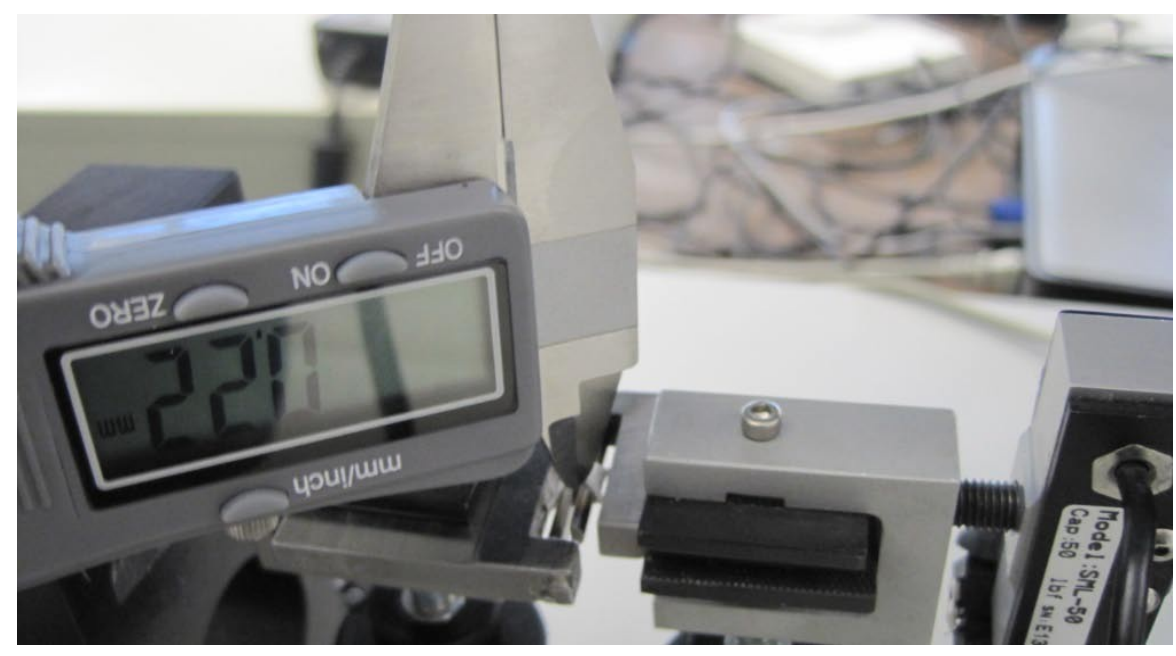

Figure 37: A sample of ePTFE being tested in tubular form using the new transverse test fixture. Calipers were used to measure the gage length of each sample.

The elastic modulus and ultimate tensile strength were determined using the same calculations as the current method with one exception. The cross-sectional area for each sample was calculated by multiplying the product of the width and thickness by two, because the sample is kept in tubular form during testing. The picture below shows a group of PLGA samples before and after testing using the new transverse test fixture. 


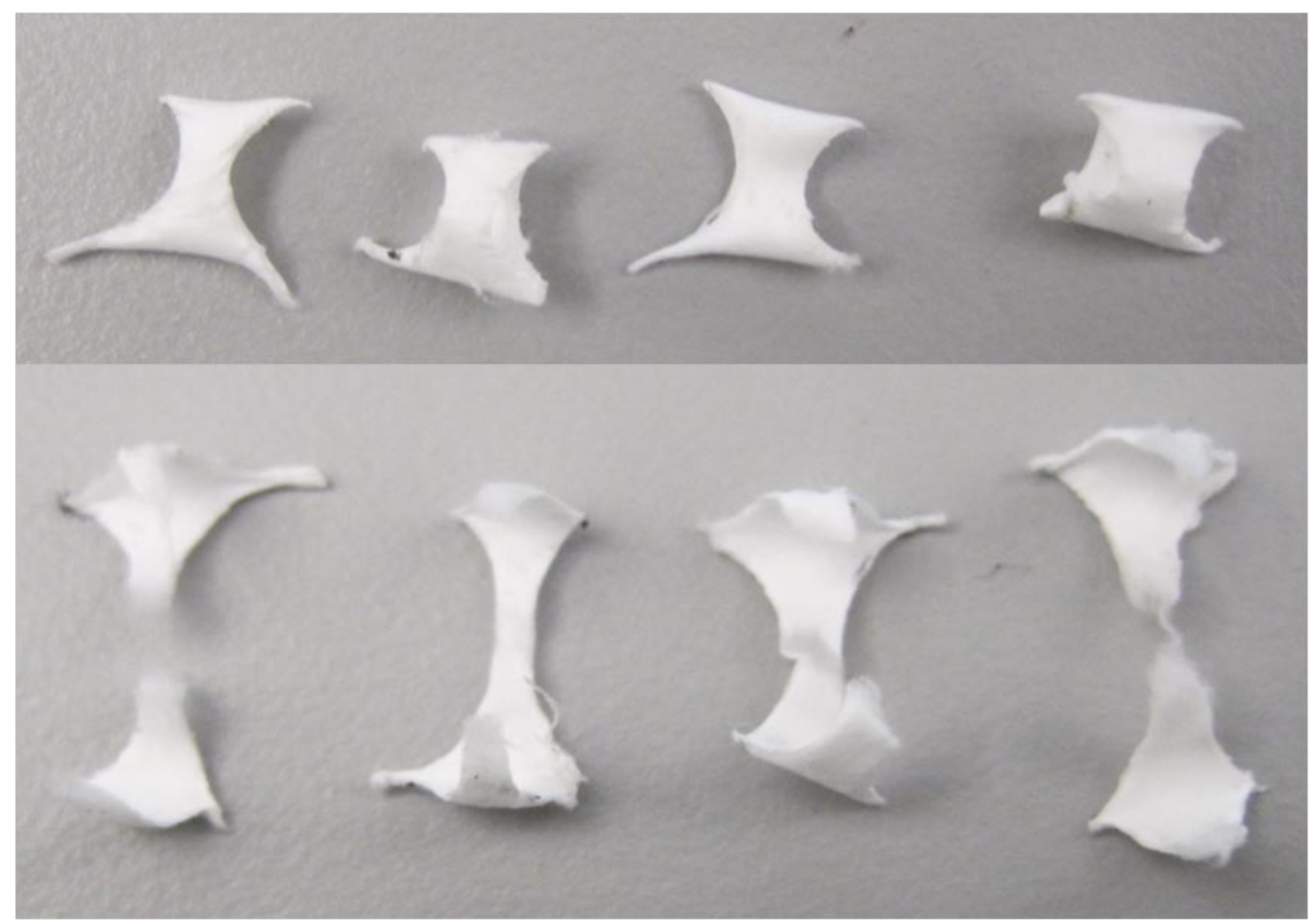

Figure 38: The top picture shows the prepared PLGA samples in tubular form. The bottom picture shows the samples after being tested. Note that for most samples tested in tubular form, one side of the tube fractured first.

Seven samples of ePTFE were tested using the transverse test fixture and four samples of ePTFE were tested using the current method of testing to perform a pilot study and power analysis. Three more samples were tested using the transverse fixture due to extra material available during the pilot study. A power analysis was performed to determine the appropriate number of samples needed to compare the two test methods in an experimental study. Four samples of PLGA were tested for each method of testing for a pilot study. The following section outlines the results of these pilot studies along with the results of the experimental study and statistical analysis to determine whether or not there was a significant difference in the two testing methods. 


\subsection{Results}

This section will present the data gathered to compare the new transverse test fixture against the current method of testing the transverse tensile properties of the tubular polymers ePTFE and PLGA. Statistical methods were used to determine whether or not there is a significant difference between the two test methods, but first a pilot study was conducted to establish an appropriate number of material samples needed in order to get accurate statistical results.

\subsubsection{Pilot Study}

A pilot study is essential to any statistical study to ensure an appropriate sample size is tested; thus, avoiding unnecessary time, costs, or materials. This also ensures that the results of the study are within a tolerable error and satisfactory level of confidence. While tolerable error is dependent on the context of the problem, a generally accepted level of confidence is $95 \%$, which is considered a reasonable level of certainty [57]. Thus, this was the level of confidence decided on for this experimental study. As mentioned in previously, four samples of ePTFE were tested using the current method of transverse tensile testing and compared to seven samples of ePTFE tested using the new transverse test fixture. Four samples of PLGA were used for each method of testing. The elastic modulus (E) and ultimate tensile strength (UTS) were calculated and analyzed separately in a power analysis using Minitab software. The following table shows the results of the ePTFE pilot study. 
Table 11: ePTFE Pilot Study Results

\begin{tabular}{|c|c|c|c|c|c|}
\hline \multicolumn{3}{|c|}{ Transverse Test Fixture } & \multicolumn{3}{|c|}{ Current Transverse Test Method } \\
\hline Sample & UTS (MPa) & $\begin{array}{l}\text { Elastic } \\
\text { Modulus } \\
\text { (MPa) }\end{array}$ & Sample & UTS (MPa) & $\begin{array}{l}\text { Elastic } \\
\text { Modulus } \\
\text { (MPa) }\end{array}$ \\
\hline 1 & 5.962 & 6.949 & 1 & 12.718 & 22.068 \\
\hline 2 & 6.105 & 5.669 & 2 & 8.023 & 16.225 \\
\hline 3 & 8.158 & 8.217 & 3 & 5.545 & 11.568 \\
\hline 4 & 7.589 & 7.023 & 4 & 12.736 & 20.842 \\
\hline 5 & 9.021 & 9.740 & \begin{tabular}{|l|} 
Average \\
\end{tabular} & 9.756 & 17.676 \\
\hline 6 & 11.300 & 9.326 & & & \\
\hline 7 & 8.863 & 7.682 & & & \\
\hline Average & 8.143 & 7.801 & & & \\
\hline
\end{tabular}

Based on the data above, the maximum difference between the means and the standard deviations were calculated for both the UTS and elastic modulus. Those values were input into Minitab’s power analysis algorithm and power curves were produced, which show the sample size needed to determine, with a $95 \%$ level of confidence, whether or not there is a significant difference between the two different methods of transverse tensile testing. The power curves below show that fifty four samples of ePTFE were needed to determine whether there is a significant difference in testing methods based on the material's UTS. The second power curve shows that only nine samples of ePTFE were needed to determine a difference based on elastic modulus. 


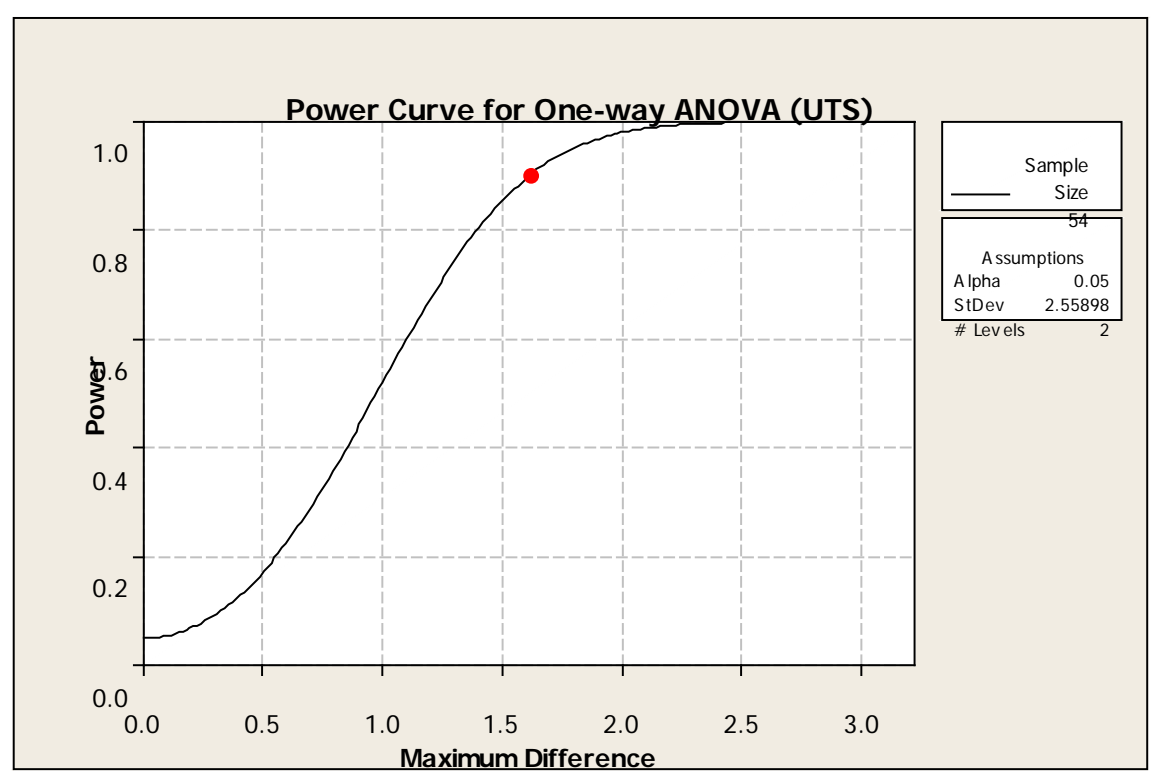

Figure 39: ePTFE power curve for pilot study. Results show a sample size of 54 is needed to determine with a $95 \%$ level of confidence if there is a main effect on UTS due to test method.

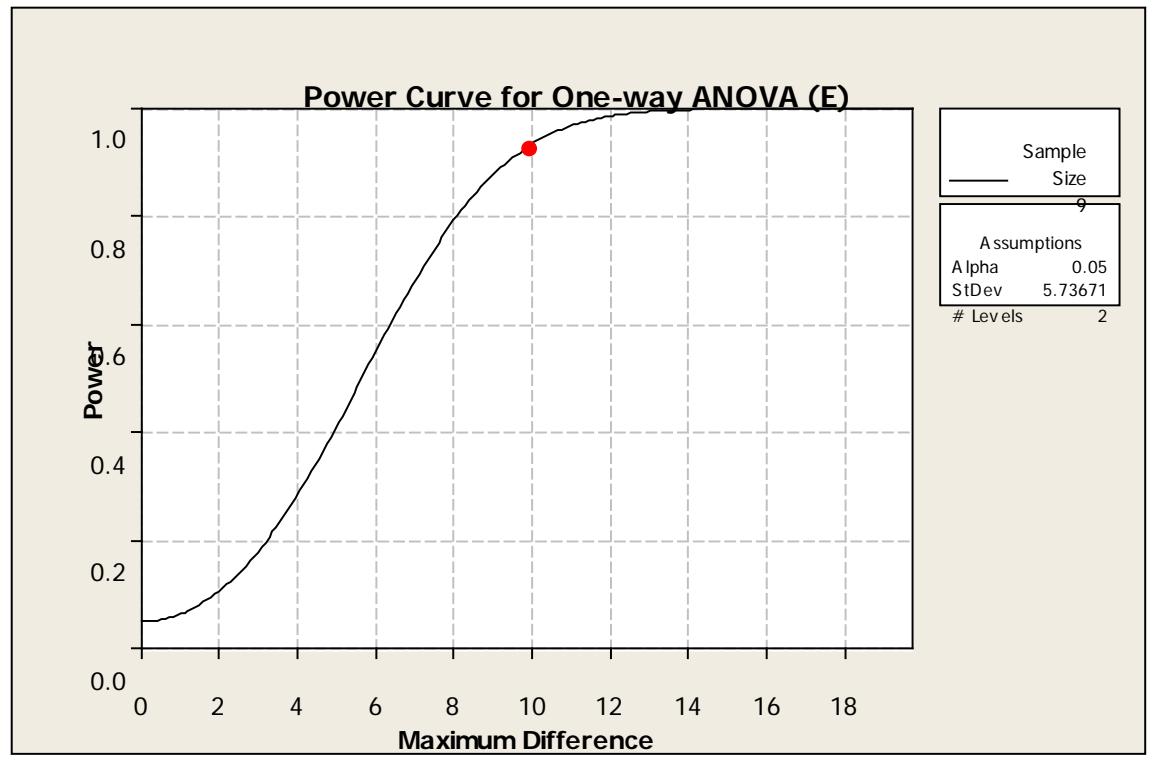

Figure 40: ePTFE power curve for pilot study. Results show a sample size of 9 is needed to determine with a $95 \%$ level of confidence if there is a main effect on elastic modulus due to test method. 
The same methods were used to calculate a sample size for the PLGA material. Below shows the data collected from the tensile tests of PLGA. The first test using the new transverse test fixture failed to record data, thus only three values for the new fixture were documented.

Table 12: PLGA Pilot Study Results

\begin{tabular}{|c|c|c|c|c|c|}
\hline \multicolumn{3}{|c|}{ Transverse Test Fixture } & \multicolumn{3}{|c|}{$\begin{array}{c}\text { Current Transverse Test } \\
\text { Method }\end{array}$} \\
\hline Sample & $\begin{array}{l}\text { UTS } \\
(\mathrm{MPa})\end{array}$ & $\mathrm{E}(\mathrm{MPa})$ & Sample & $\begin{array}{l}\text { UTS } \\
(\mathrm{MPa})\end{array}$ & $\mathrm{E}(\mathrm{MPa})$ \\
\hline 2 & 2.784 & 4.174 & 1 & 2.693 & 72.893 \\
\hline 3 & 2.169 & 31.613 & 2 & 2.794 & 34.649 \\
\hline 4 & 2.536 & 3.966 & 3 & 2.942 & 12.340 \\
\hline Average & 2.496 & 13.251 & 4 & 2.710 & 64.515 \\
\hline & & & Average & 2.785 & 46.099 \\
\hline
\end{tabular}

After calculating the difference between means and the standard deviations for both UTS and elastic modulus, the data was input into Minitab to produce a power curve. It was determined that a sample size of seventeen would show if there was a significant difference between the two testing methods based on both UTS and elastic modulus. This determination was based on a $95 \%$ level of confidence. Below shows the two power curves based on UTS or elastic modulus. 


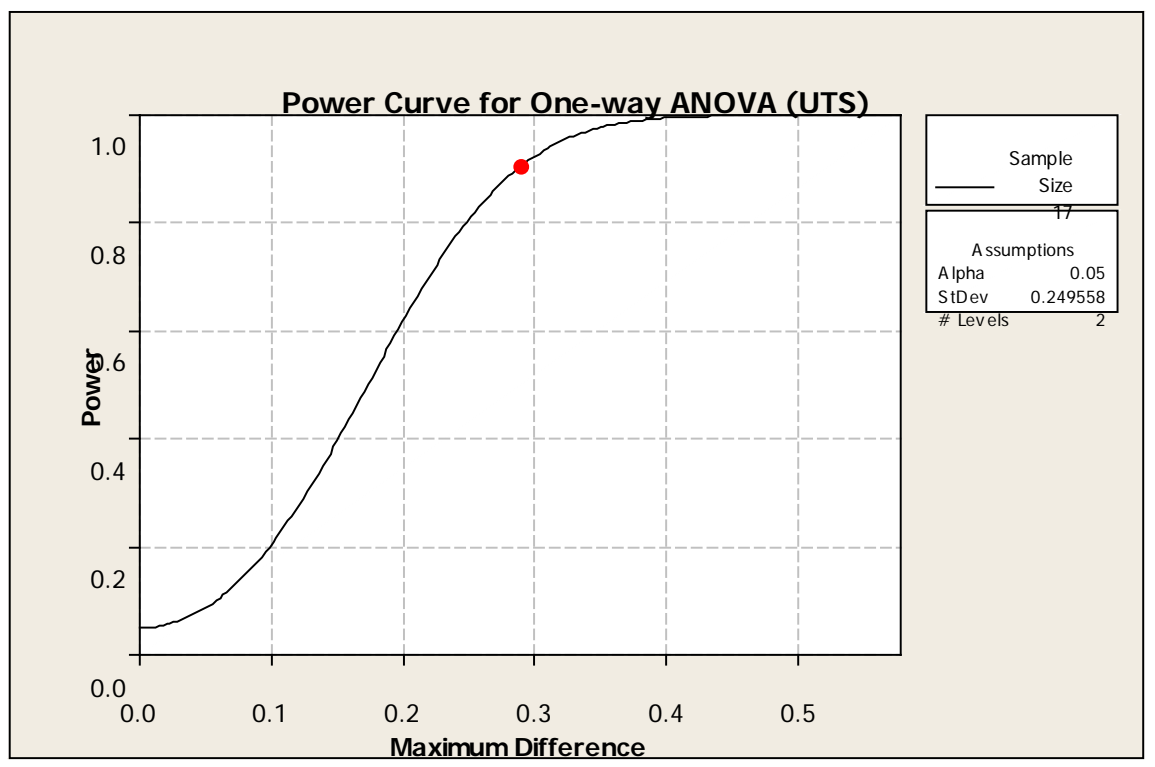

Figure 41: PLGA power curve for pilot study. Results show a sample size of 17 is needed to determine with a $95 \%$ level of confidence if there is a main effect on UTS due to test method.

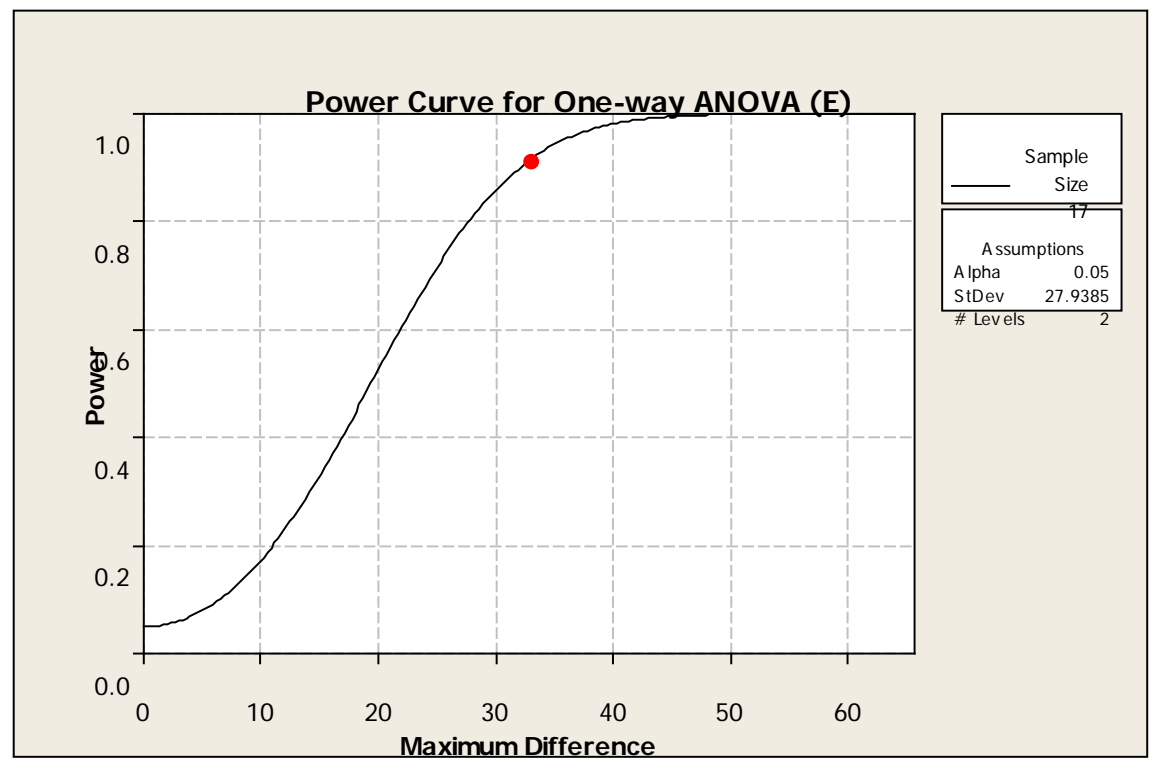

Figure 42: PLGA power curve for pilot study. Results show a sample size of 17 is needed to determine with a $95 \%$ level of confidence if there is a main effect on elastic modulus due to test method. 
Based on the cost and availability of ePTFE, fifty four samples was an unrealistic number for a sample size. Because nine samples were considered sufficient to determine whether or not there is a difference between testing methods based on elastic modulus, this was the sample size chosen for the experimental study. For a sample size of nine to determine a significant difference in test methods for ePTFE, only a 75\% level of confidence could be reached for UTS. Seventeen was a realistic sample size for PLGA due to its availability from Cal Poly's tissue engineering group. The following section will explain in detail the design of experiments including objectives, hypotheses, and treatment and design structure.

\subsubsection{Design of Experiments}

The objective of this statistical analysis was to determine, with a $95 \%$ confidence, if there is a significant difference in testing the transverse tensile properties of tubular material specimens using two different testing methods: testing a sample in tubular form using the newly developed test fixture as opposed to the current method of testing a sample that is cut and laid flat. Both the ultimate tensile strength and elastic modulus of the materials were sampled for PLGA and ePTFE. The research hypothesis was outlined as follows:

$$
\mathrm{H}_{0}: \mu_{\mathrm{TF}}=\mu_{\mathrm{CM}} \mathrm{H}_{\mathrm{A}}: \mu_{\mathrm{TF}} \neq \mu_{\mathrm{CM}}
$$

$\mu_{\mathrm{TF}}$ symbolizes the average ultimate tensile strength or elastic modulus of all material samples tested using the new transverse test fixture, while $\mu_{\mathrm{CM}}$ represents the average ultimate tensile strength or elastic modulus of all material samples tested using the current method of testing. 
The treatment structure for this experiment was set up as "test method" at two levels: testing the material in tubular form as opposed to cutting the tube and laying it flat. The two response variables were ultimate tensile strength and elastic modulus, with a single test sample of material being the experimental unit.

The design structure was to be completely randomized; however, the lack of material availability offered limitations to this design. The material provided came in long tubes that were cut down to sample size using the methods described in section 4.2.2. Because all of the ePTFE samples came from the same length of tubing, conclusions can be made for that batch of material, but are limited as to the population of ePTFE. Likewise, the majority of samples of PLGA came from only two different electrospinning sessions; thus, an assumption as to the population of PLGA cannot be made with confidence. Other sources of variation in this experimental design that should be noted include, but are not limited to the preparation of each experimental unit, measurement of the thickness, width, and gage length of each sample, and material fabrication (PLGA). All statistical analyses were performed using Minitab software. The next section presents the results of the experimental study, beginning with the results for ePTFE.

\subsubsection{Results-ePTFE}

A total of nine samples of ePTFE were tested using the current method, and eight results were recorded due to an error on the PDA. Of the eight recorded results, two samples of ePTFE never fractured because they slipped out of the Instron grips, thus counting that data null and void. For the ePTFE tested in tubular form, eight samples 
were tested due to material availability; however, only seven tests were recorded due to one sample breaking before test could be conducted. The results for all ePTFE testing, including each sample's cross-sectional area, ultimate tensile strength, and elastic modulus, may be found in Appendix F. After testing, a one-way analysis of variance (ANOVA) test was analyzed for both elastic modulus and ultimate tensile strength. Three assumptions must be made when using an ANOVA test: samples come from normally distributed populations, variances are equal, and samples are independent. The following residual plots confirm these assumptions.

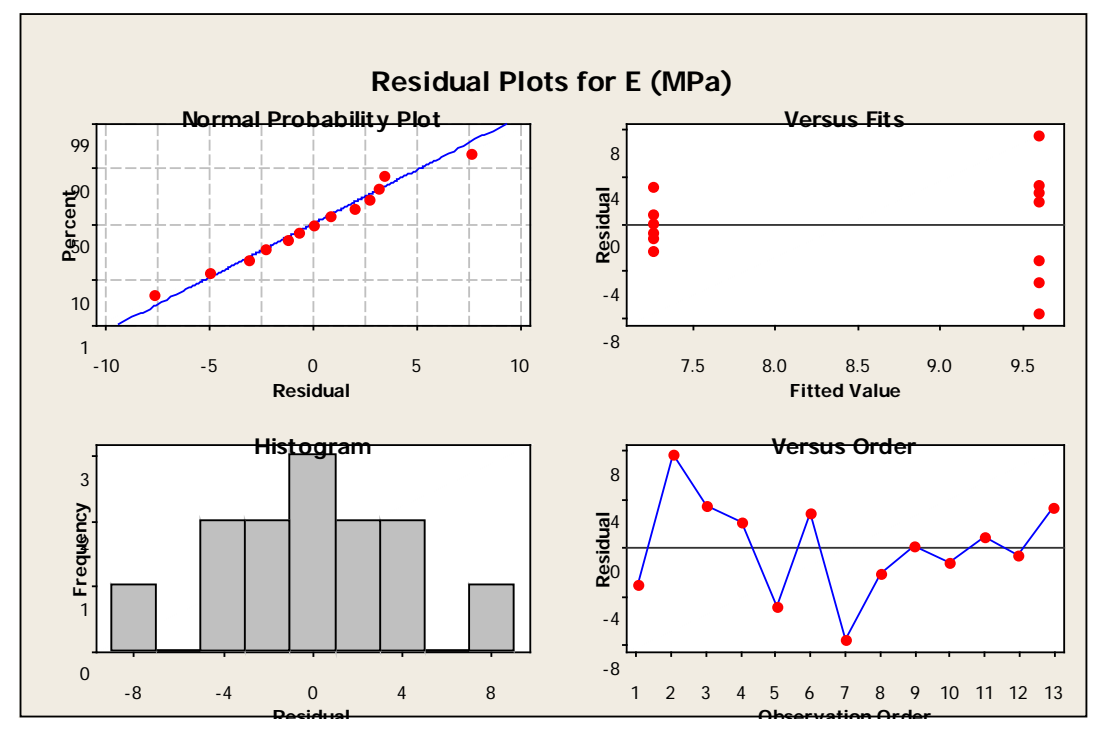

Figure 43: One-way ANOVA residual plots for ePTFE elastic modulus. Plots show normally distributed populations and variances within three times of each other. 


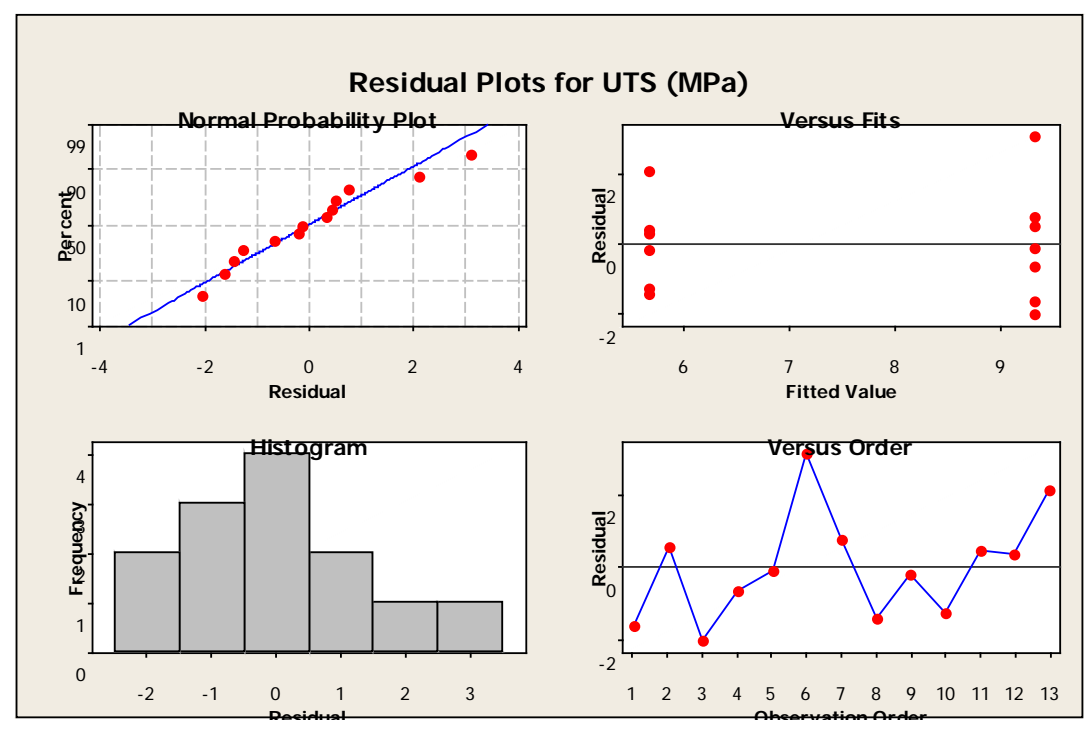

Figure 44: One-way ANOVA residual plots for ePTFE UTS. Plots show normally distributed population and variances within three times of each other.

In both normal probability plots for elastic modulus and UTS, there are no extreme outliers, which confirm normally distributed populations. In the versus fits plot, both populations for elastic modulus and UTS show variances within three times of each other, thus we can assume equal variances. A completely randomized design assures independent populations, which for this experimental study, does have some limitations as discussed above.

The one-way ANOVA test for UTS using Minitab software resulted in a p-value of 0.001 . This is less than our significance level of the test $(\alpha=0.05)$, thus $\mathrm{H}_{0}$ was rejected. This concludes that there is a significant difference between test methods on the ultimate tensile strength of ePTFE. Below are the results of the one-way ANOVA study. 


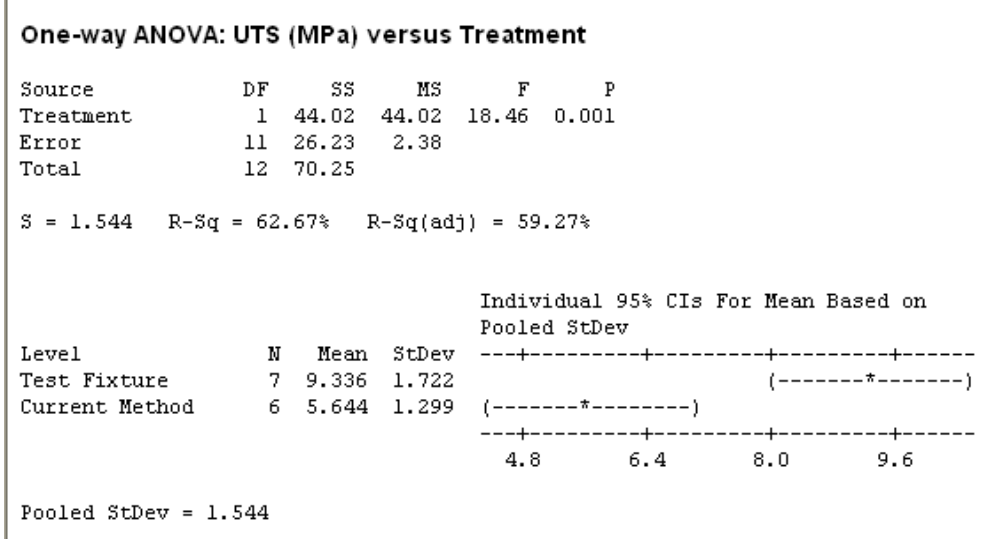

Figure 45: ePTFE one-way ANOVA results for UTS. $P=0.001<0.05=\alpha$, thus $\mathrm{H}_{0}$ is rejected.

The outcome of the one-way ANOVA for the elastic modulus of ePTFE resulted in a p-value of 0.333 , which is greater than the significance level of the test $(\alpha=0.05)$, thus the test failed to reject $\mathrm{H}_{0}$. This shows that there is not sufficient evidence to suggest a difference between test methods for the elastic modulus of ePTFE. Below are the results of the one-way ANOVA experimental study.

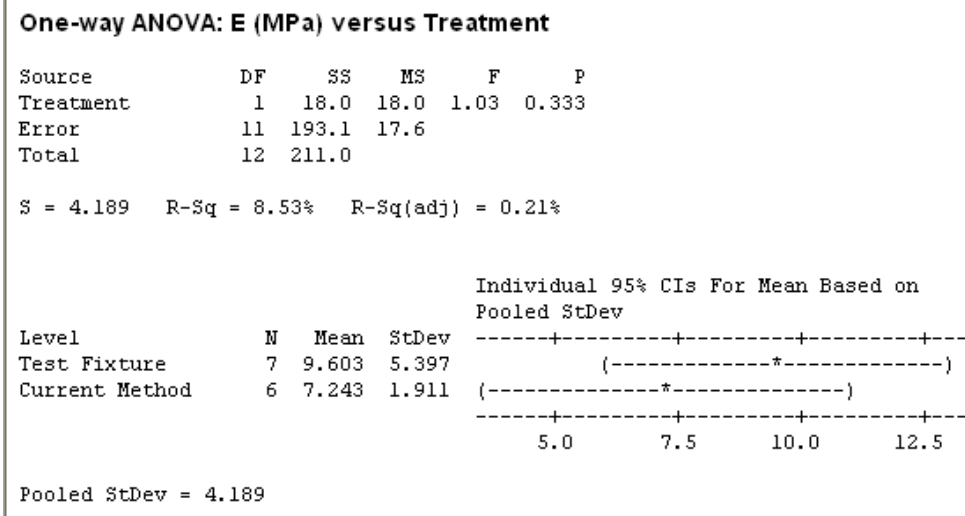

Pooled StDev $=4.189$

Figure 46: ePTFE one-way ANOVA results for elastic modulus. $P=0.333>0.05=$ $\alpha$, thus the test failed to reject $\mathrm{H}_{0}$. 


\subsubsection{Results-PLGA}

Seventeen samples of PLGA were tested and results were recorded using the current method of testing. Due to material availability, however, only fourteen samples of PLGA were tested and results recorded using the new method of testing where the material was kept in tubular form. The results of all PLGA testing can be found in Appendix F, including each sample’s cross-sectional area, ultimate tensile strength, and elastic modulus. Below are the residual plots for the two response variables (elastic modulus and ultimate tensile strength) compared at the two testing levels (current method vs. transverse test fixture).

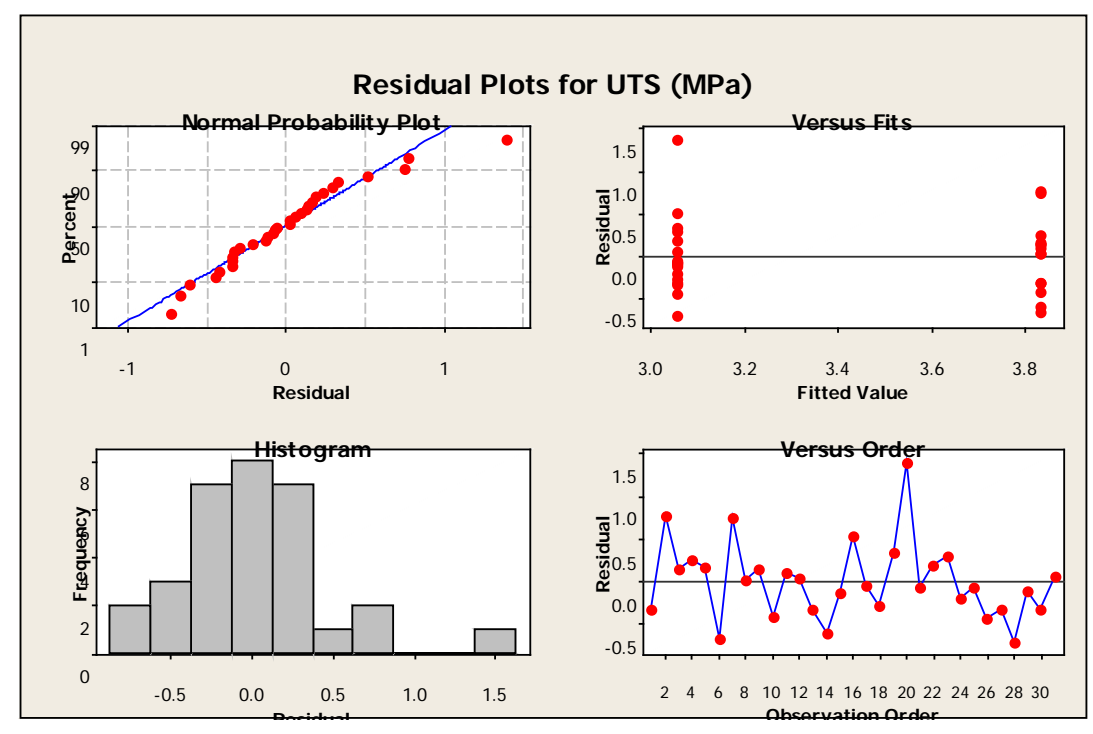

Figure 47: One-way ANOVA residual plots for PLGA UTS. Plots show normal population distribution and variances within three times of each other. 


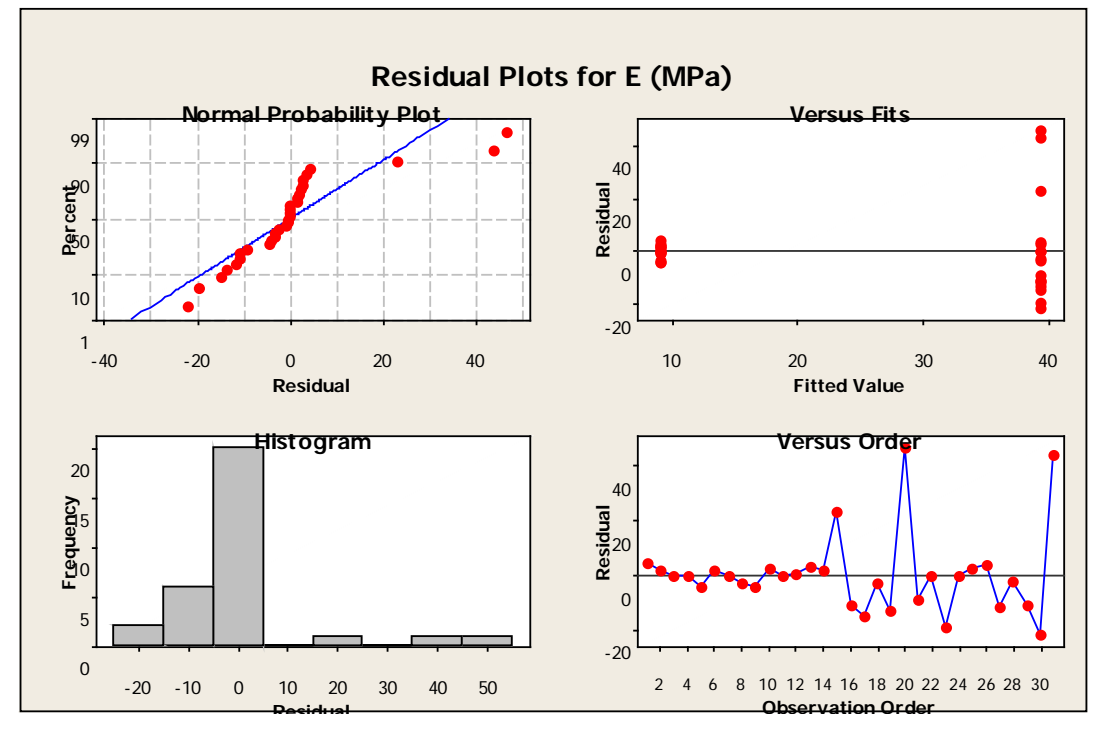

Figure 48: One-way ANOVA residual plots for PLGA elastic modulus. Plots do not show normal population distribution and variances are unequal.

In the residual plots for UTS, the normal probability plot and versus fits plot show a normally distributed population and equal variances for PLGA. The residual plots for elastic modulus, on the other hand show a number of outliers in the normal probability plot, and the versus fits plot does not suggest equal variances. While a one-way ANOVA study was carried out for the elastic modulus of PLGA and the results were recorded below, this study lacks a valid conclusion because the assumptions were not met.

The one-way ANOVA study comparing the effects of test method on the ultimate tensile strength of PLGA produced a p-value of 0.000 , which is less than the significance level of the test $(\alpha=0.05)$. This means $\mathrm{H}_{0}$ was rejected, and it was concluded that there is a significant difference between test methods on the ultimate tensile strength of PLGA. The following figure displays the results of the one-way ANOVA study. 


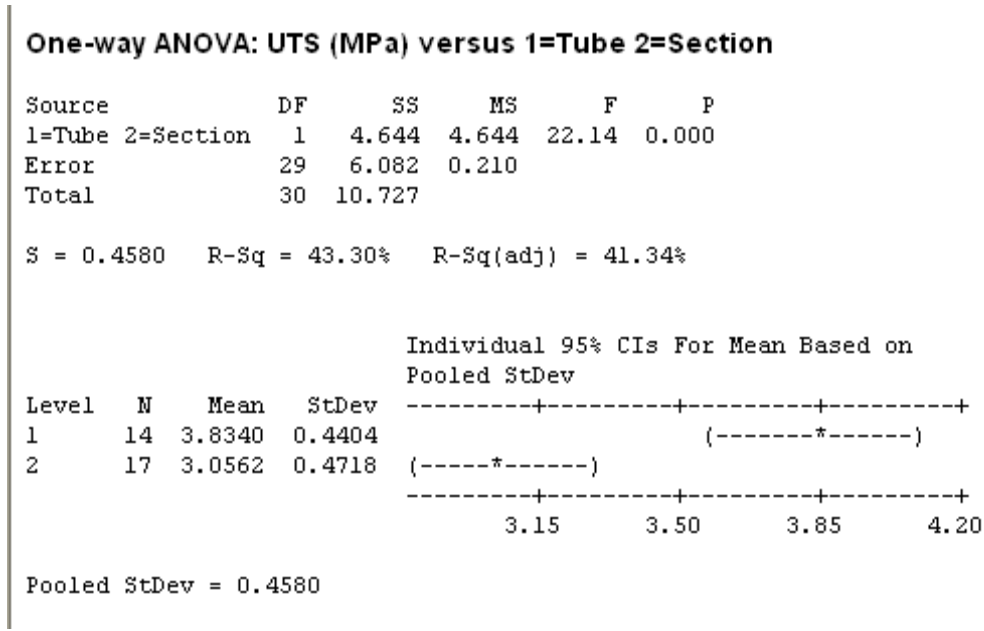

Figure 49: PLGA one-way ANOVA results for UTS. $P=0.000<0.05=\alpha$, thus $H_{0}$ is rejected.

The same one-way ANOVA test was used to determine if there was a main effect on elastic modulus of PLGA due to test method. The experimental study resulted in a pvalue of 0.000 , which would normally lead to the conclusion to reject $\mathrm{H}_{0}$; however, this is not a valid conclusion because the assumptions were not met, as discussed previously. Below are the results of the one-way ANOVA study for the elastic modulus of PLGA. 


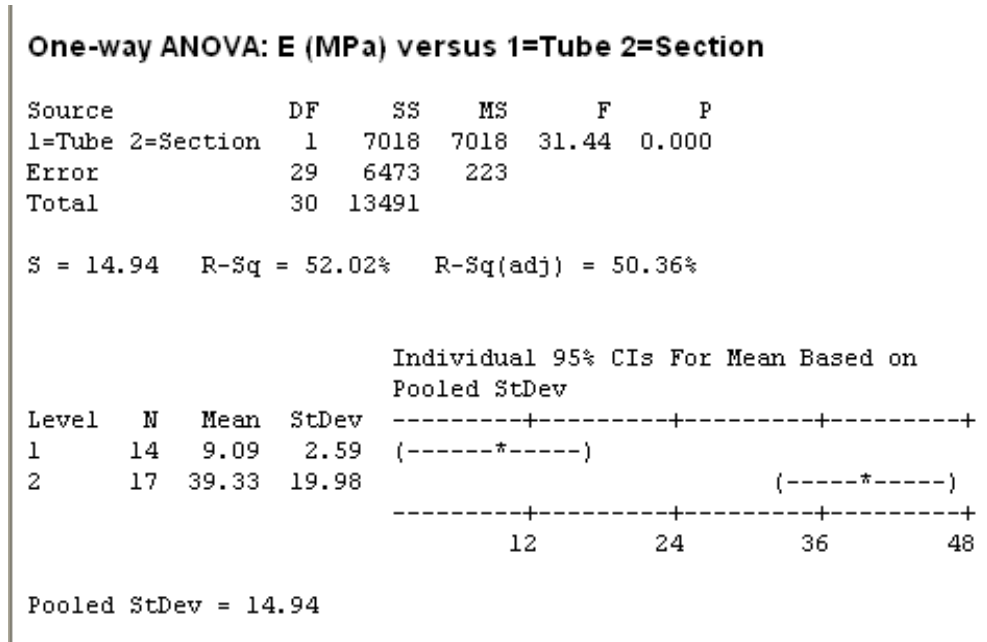

Figure 50: PLGA one-way ANOVA results for elastic modulus. These results, however, are discounted due to unequal variances.

\subsubsection{Summary of Results}

In summary, we can conclude that there was a main effect on ultimate tensile strength for both ePTFE and PLGA due to test method. This shows there is a difference in test methods when testing for ultimate tensile strength, but this analysis cannot conclude which method is more accurate. Additionally, there was no main effect on elastic modulus for ePTFE due to test method, and no conclusions could be made as to the effect on elastic modulus for PLGA due to the failure to meet the assumptions for an ANOVA test. More testing is needed to clarify the effects on elastic modulus due to test method for PLGA. Through testing the ultimate tensile strength of both materials, however, the data provided demonstrated a significant difference in test method, thus a step forward in developing a standard transverse tensile test method for small diameter tubular polymers. Chapter 6 will discuss these results in detail, but first, Chapter 5 will provide an overview of the design and analysis of the longitudinal test fixture. 


\section{Chapter 5 - Design of Longitudinal Test Fixture}

\subsection{Overview}

The ideation and design of the longitudinal test fixture for this thesis was performed in collaboration with John Anderson, a Biomedical Engineering undergraduate student, in order to fulfill requirements for his senior project. This chapter will summarize John's work, including an outline of the design requirements, a description of initial design iterations, and finally, the final longitudinal test fixture design.

Currently, in order to test the tensile properties of a tubular material with the Biomedical Engineering department's Instron In-Spec 2200, the material is cut then laid flat between the Instron grips and pulled apart in the longitudinal direction, similar to how the transverse tensile properties are tested as discussed in Chapter 4. Below shows a diagram of how a typical, tubular material would be set up for testing.

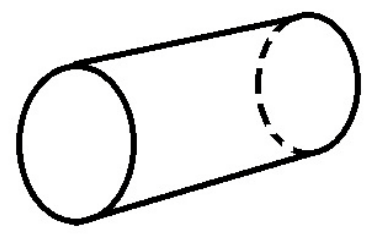

$\underline{\text { Tubular Form }}$

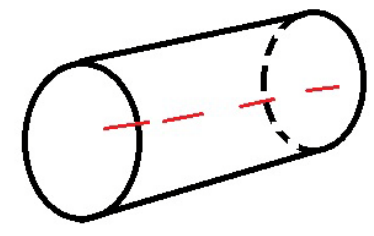

Cut is made along the red line

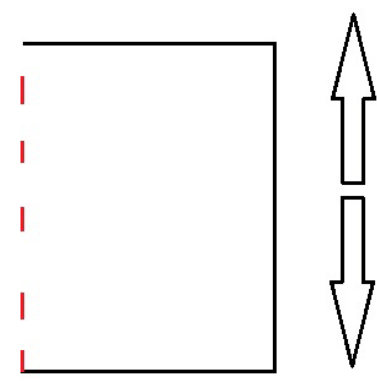

Polymer Layed Flat and gripped at ends

Figure 51: In order to test the tensile properties of tubular materials in the longitudinal direction, the samples are prepared by cutting along the length of the tube and laying the piece flat between the Instron grips as diagrammed above. 
Similar to the transverse test fixture, the goal of the final longitudinal test fixture was for it to be tested with the Instron testing machine while keeping the material test sample in tubular form. The following section will explain more specific design criterion.

\subsection{Design Requirements}

In order for the final longitudinal test fixture to be compatible with the Instron InSpec 2200 testing machine, it must meet specific size criterion to fit in the Instron grips, as discussed previously regarding the transverse test fixture. Another design requirement that was specified was for an inner support structure to keep the material in tubular form. This would also keep the sample from flattening when inserted into the Instron grips. The third criteria for the longitudinal test fixture design required a cylindrical interface around the ends of each material sample. Currently the grips on the Instron machine are flat and have small jaws in order to securely hold a material sample. These jaws, however, can apply excessive shearing to the material that may result in material tearing at the grip interface, especially with an elastic material such as ePTFE. Therefore, a cynlindrical, smooth interface that will securely hold the test sample in the Instron grips will keep the material in tubular form as well as prevent tearing at the grips. With these design requirements in mind, the next section will briefly describe the three initial design iterations leading up to the final design for the longitudinal test fixture. 


\subsection{Initial Design Iterations}

\subsubsection{Initial Design 1}

The first design for the longitudinal test fixture included two identical components for each end of the tubular test sample that would fit into the Instron grips. Each component was comprised of a central piece: one end was a flat slab that would be secured in the Instron grips and the other end was a mandrel that would fit into the tubular sample. Two surrounding half cylinder pieces would then be secured over the test sample to hold it in place during testing. The half cylinder pieces would be held in place by an attached threaded component fitting through the back of the central piece and secured with a nut. A picture of the rapid prototyped design is shown in the figure below.
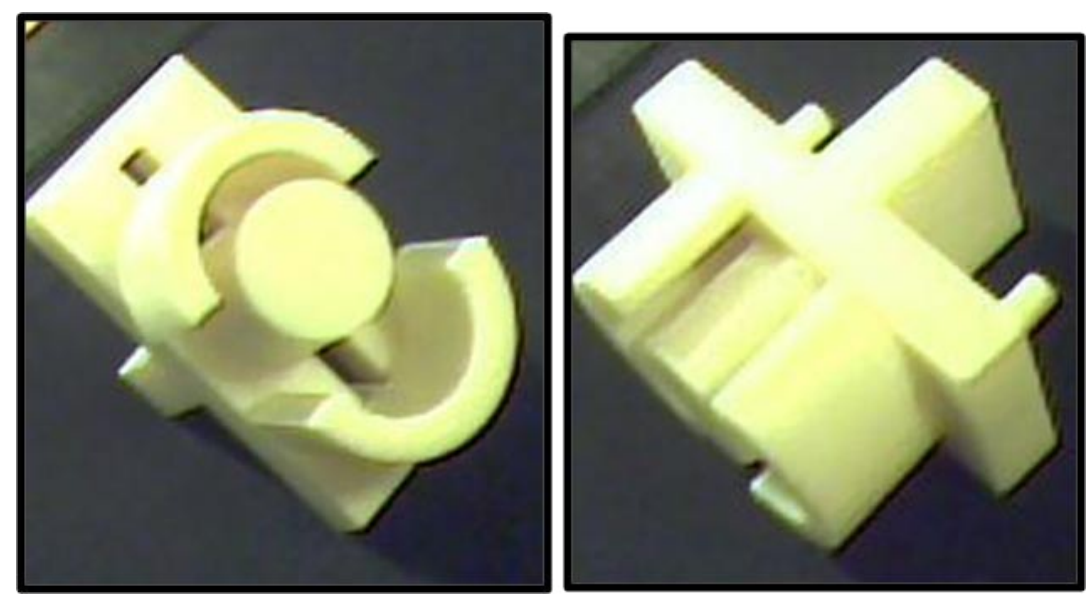

Figure 52: Rapid prototyped model of the first design iteration.

While the ease of use for this design was excellent, the manufacturability would be extremely difficult to produce to a scale that would match ePTFE or PLGA samples. Another limitation to this design was the inability of the surround half cylindrical parts to successfully hold the material sample during testing. Because the surrounding parts were 
secured laterally to the fixture, there was not enough vertical pressure to clamp the test sample while it was being pulled in the lateral direction. From this design, it was clear that the next iteration must be easier to manufacture as well as provide a more secure grip on the tubular material sample during testing.

\subsubsection{Initial Design 2}

With the idea of ease of manufacturing in mind, the second design iteration for the longitudinal test fixture incorporated a mandrel that would fit inside each end of the tubular material sample. Each end would then be surrounded by two blocks that each had a semi circular notch cut out in order to secure the sample. Each set of blocks would then fit into the Instron grips to provide pressure to the tube in order to hold it in place during testing. The rapid prototyped model of this design iteration is pictured below.

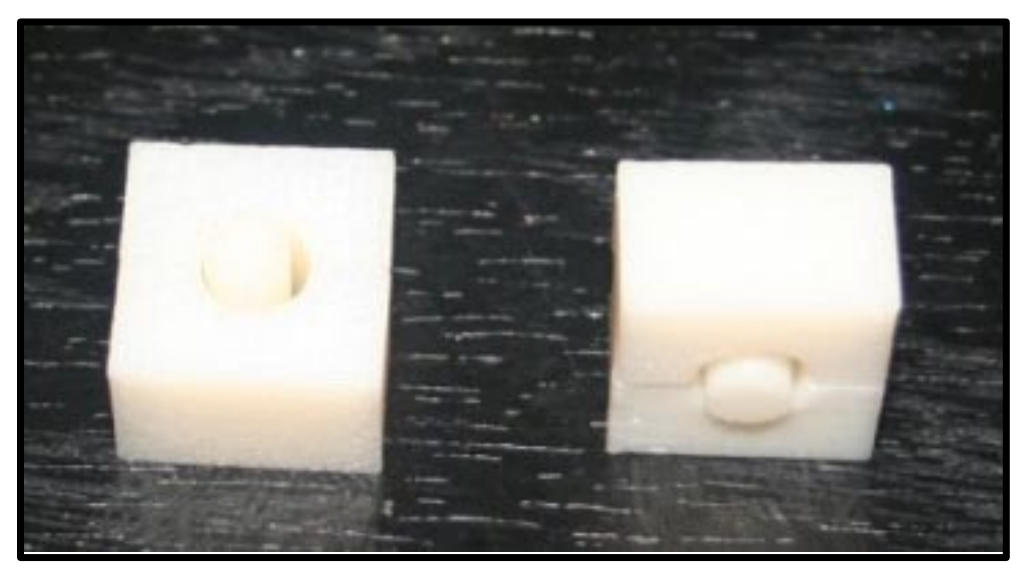

Figure 53: Rapid prototyped model of the second design iteration.

While the simplicity of this design would make the fixture easy to manufacture, the rapid prototyped model uncovered some design limitations. Because there was nothing holding the three components of this fixture together, it was hard to align the 
material within the fixture and then secure it to the Instron grips. The ease of handling this longitudinal test fixture design was poor; thus, it was deemed that the mandrel should be somehow attached to the part of the fixture that is secured to the Instron grips, similar to the first design iteration. The following section will describe how the third initial design addressed this handling issue.

\subsubsection{Initial Design 3}

The major change to the longitudinal test fixture in the third design iteration was a back plate fixed to the end of each mandrel for ease of handling when testing tubular samples. To load a test sample onto the mandrel, the user could simply hold the back plate in order to stabilize the test fixture. The surrounding blocks, which remained the same from the second design iteration, were then easily placed around the mandrel with the back plate causing no interference. The rapid prototype of the third design iteration is shown in the figure below.

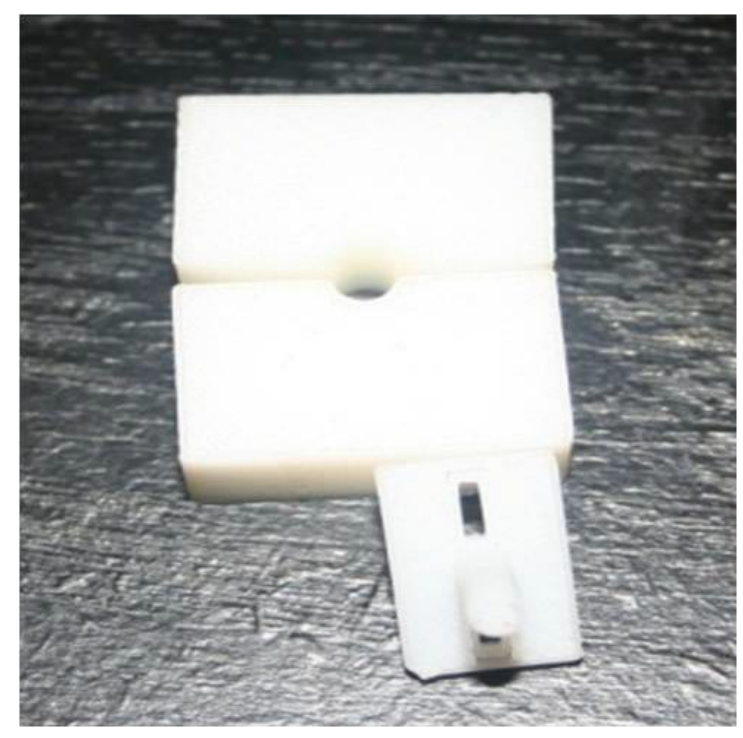

Figure 54: Rapid prototyped model of the third design iteration. 
Because the third design iteration was mainly focused on ease of use, no design changes were made to the blocks used to surround and support the test sample around each mandrel. It was discovered through testing in the Instron In-Spec grips, however, that the blocks did not apply enough pressure to the test sample and mandrel to keep the sample from slipping out of the grips during testing. This obstacle was overcome in the final design iteration that is described in the next section.

\subsection{Final Design of Longitudinal Test Fixture}

The final design for the longitudinal test fixture incorporated many aspects of previous design iterations, but combined them in a way as to overcome all previous design challenges. The mandrel attached to a back plate remained the same from the third design iteration. The blocks that surrounded the mandrel from the third design, however, failed to secure the test sample around the mandrel to prevent the sample from slipping out of the fixture during testing. In order to apply enough pressure to the test sample around the mandrel, the final design integrated the nut and bolt tightening feature from the first design iteration with a slight difference. In the first design iteration, the nut and bolt feature tightened the fixture together laterally, but in the final design, the nut and bolt was repositioned vertically on the fixture. This provided more pressure to the test sample in order to prevent it from slipping out of the mandrel during testing. The figure below shows the Solidworks model of both the mandrel and the top portion of the test fixture that surrounds the test sample on the mandrel. 

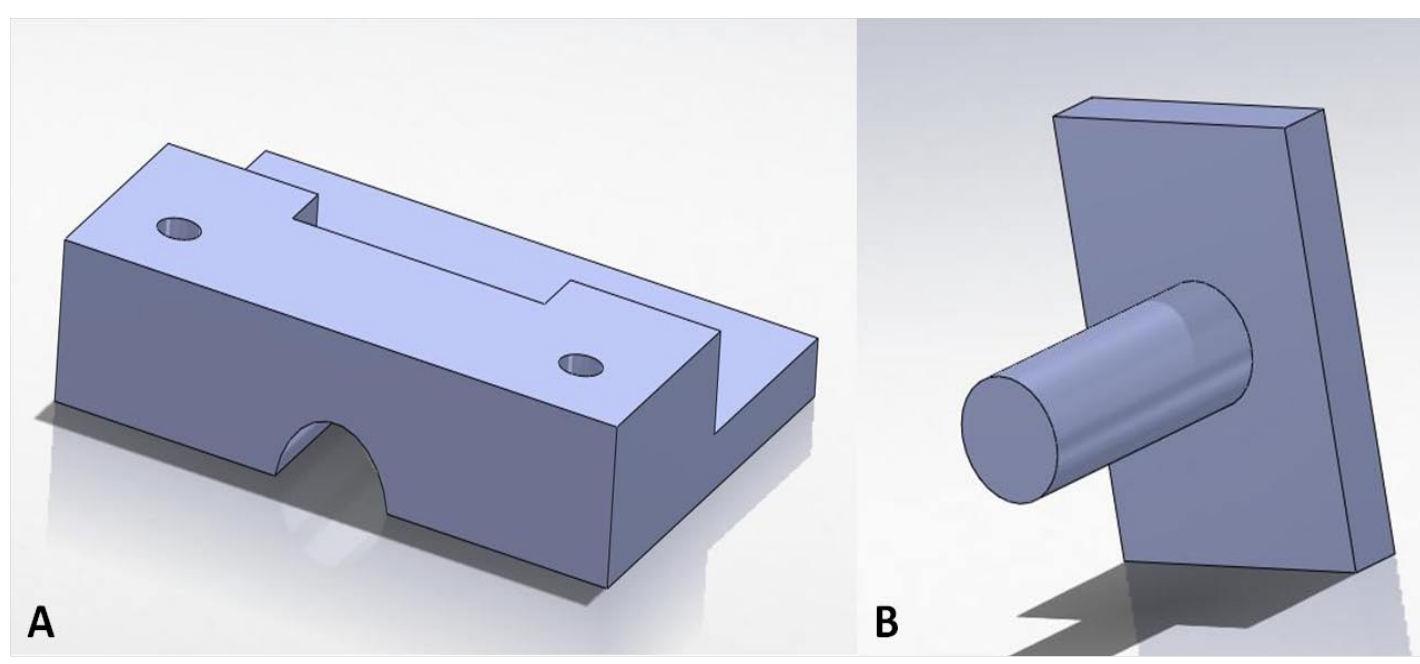

Figure 55: A) Solidworks model of the top portion of the longitudinal test fixture, with a semicircular notch cut out on the bottom and two holes placed on top for the screws to be secured. B) Solidworks model of the mandrel attached to the back plate, which slides inside the slot made on the top and bottom portion of the fixture.

The top and bottom parts of the fixture that would surround the mandrel consisted of a thin back half of the piece that would fit into the Instron In-Spec grips connected to a thicker front half of the piece that would protrude out of the grips in order to fit the two screws that would secure the fixture around the test sample. Between the thinner back half and thicker front half, a slot was placed to accommodate the back plate attached to the mandrel. This would further assist in handling the fixture during tensile testing, while not compromising any of the other design requirements. The same semicircular notch from the second and third design iteration was used in the top and bottom parts of the fixture to encompass the test sample around the mandrel while keeping it as close to its natural tubular form as possible. Two holes were placed on each side of the semicircular notch on both the top and bottom parts. The top part was to be machined as a through hole, where as the bottom part was a threaded hole. This allowed for two screws to be 
easily placed in the top part of the fixture then secured to the bottom part by the threads, thereby securing the fixture around the test sample and the mandrel. The detailed drawings for final test fixture can be found in Appendix G.

The final test fixture was manufactured at Precision Machine Shop in San Luis Obispo. The same mild steel beam used for the transverse test fixture was used to machine the longitudinal test fixture parts. The figure below shows the final manufactured longitudinal test fixture.

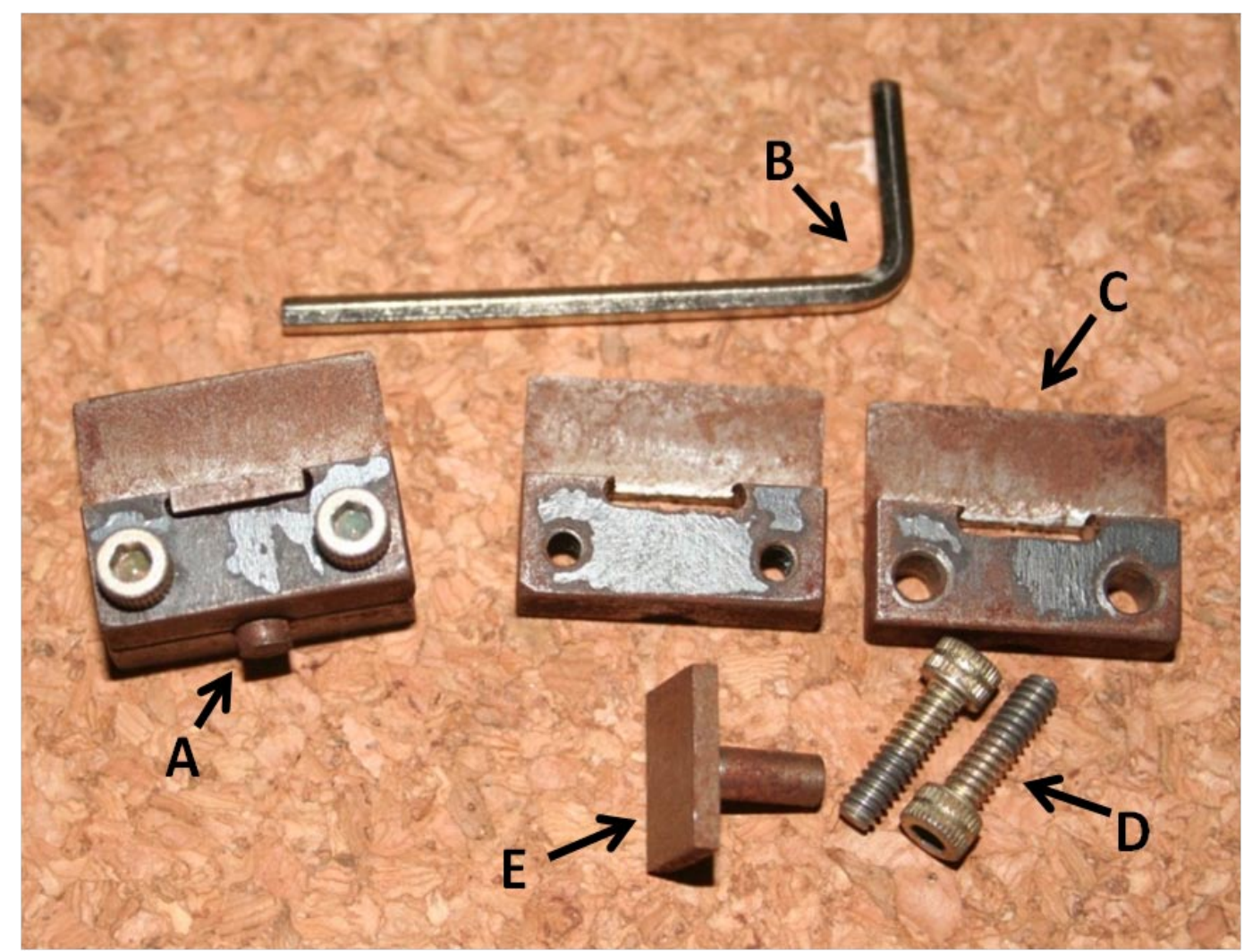

Figure 56: A) Final manufactured longitudinal test fixture; B) L-wrench used to tighten screws onto fixture; C) Top portion of fixture; D) Screws used to secure top and bottom portions around mandrel; E) Mandrel connected to back plate. 
The following section will show how the longitudinal test fixture is used to tensile test small diameter tubular polymers in the Instron In-Spec machine, and provide preliminary data in testing the efficacy of this fixture compared to the current method of testing.

\subsubsection{Testing the Final Longitudinal Test Fixture}

Material preparation and testing for the longitudinal test fixture was simple and straight forward. While PLGA was the only material tested using the newly designed fixture, it is intended to be used for ePTFE as well as other small diameter tubular polymers. The PLGA samples, which came in long lengths of tubing, were cut into 10$15 \mathrm{~mm}$ length test samples. The wall thickness and width of the tubular samples were measured using calipers before being loaded into the test fixture. The width of each sample was measured as the tube's diameter. The sample was then loaded onto the mandrel and positioned into the top and bottom parts of the fixture. The screws were tightened to secure the test sample in place then the entire fixture was loaded into the Instron grips. The gage length of the sample was measured as the distance between each of the opposing fixtures after being loaded into the Instron machine. Figure 57 below, shows a test sample of PLGA secured in the test fixture and loaded into the Instron grips. 


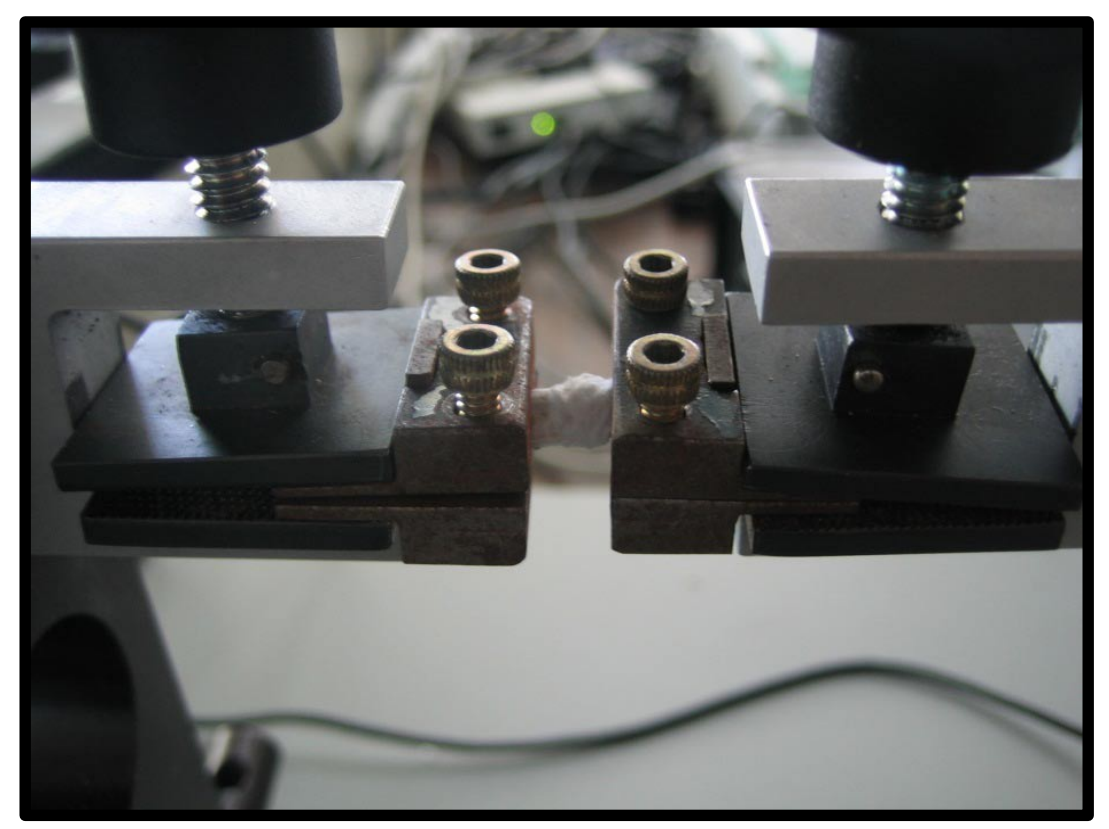

Figure 57: Tubular sample of PLGA secured in test fixture and loaded onto Instron grips, ready to be tested.

A total of six samples of PLGA were tested using the newly designed test fixture, and the ultimate tensile strength was recorded for each test. In all six tests, none of the samples ever slipped out of the test fixture, and a clean, mid-section tear was accomplished in every sample. The table below summarizes the results. 
Table 13: UTS results from testing six samples of PLGA in tubular form using the final longitudinal test fixture.

\begin{tabular}{|c|c|}
\hline Sample & Ultimate Tensile Strength (Mpa) \\
\hline 1 & 1.571 \\
\hline 2 & 2.934 \\
\hline 3 & 4.285 \\
\hline 4 & 6.413 \\
\hline 5 & 6.322 \\
\hline 6 & 7.35 \\
\hline
\end{tabular}

While the results of the preliminary testing of the longitudinal test fixture did not offer a comparison to the current method of testing, it did show the feasibility of using the fixture, and somewhat consistent ultimate tensile strength data. The following section will provide a discussion of John's work including design limitations and suggestions as to how this longitudinal test fixture design could be improved or expanded upon.

\subsection{Discussion}

Through research, conceptualization, and assessment, a fixture was created that would successfully test the longitudinal tensile properties of small diameter tubular polymers while maintaining the test sample in tubular form. The design was able to meet the requirements outlined previously that included the following: the fixture must be compatible with the Instron In-Spec tensile testing machine; the test sample must remain in tubular form requiring an inner mandrel on the fixture to prevent the tube from collapsing on itself; and lastly, a cylindrical, smooth surface surrounding the test sample 
in order to secure it to the fixture without causing tearing at the grips. There were some limitations, however, which will be addressed in the following paragraphs.

A major limitation to the design process was that the first 3 design iterations were not modeled to scale making it difficult to assess the true functionality of the fixture. The first initial design was modeled to be rapid prototyped to scale; however, manufacturability was an issue as discussed previously. In the second design iteration, the parts for the fixture were simple and deemed easy to manufacture, yet were still modeled on a larger scale. The second initial design's rapid prototyped part was too large to fit a sample of PLGA or fit in the Instron grips. While it was shown that this design was not user friendly, it was difficult to assess the functionality of the oversized model.

Because the second design iteration was immediately dismissed due to ease of use issues, it was overlooked that the blocks surrounding the mandrel would not apply enough pressure needed to secure the test sample to the fixture during testing. Thus, it took a third design iteration to uncover this problem. Eventually, John was able to overcome all design challenges in the final design; however, in an industry setting, more design iterations can become extremely costly and time consuming. By modeling each design iteration to the appropriate scale, a more complete assessment of each design would be able to be accomplished.

While the final longitudinal test fixture met the initial design criteria, there was no extensive testing of the final test fixture nor was a statistical analysis conducted. In order to further analyze the validity of the design, it would be beneficial to compare tensile strengths of material tested using the new fixture compared to the current method of 
testing, much like the ANOVA studies documented in Chapter 4. Testing another material, such as ePTFE, would allow further assessment of the test fixture as well. In summary, John was successful in creating a fixture that would test the longitudinal tensile properties of small diameter tubular polymers while keeping the test sample in tubular form. The following chapter is meant to provide an in depth discussion of the results of testing the transverse test fixture, which also applies to future work with the longitudinal fixture, as well as explain the limitations of the experimental study and offer suggestions for future work to expand on this thesis for both test fixtures. 


\section{Chapter 6 - Discussion and Conclusions}

\subsection{Overview}

As the incidence of coronary and peripheral artery disease continues to grow, there continues to be a search for optimal treatment and replacement of diseased vessels [1]. Vascular bypass grafts are a popular option; however, there has yet to be a "gold standard” for a biomaterial that will mimic the mechanical properties of native blood vessels [2]. Furthermore, as different materials are researched to replace diseased vessels, methods of testing the tensile properties of these potential grafts have been inconsistent. This has made comparing documented tensile strengths of small diameter tubular polymers to native vessel strengths difficult. Thus, the aim of this thesis was to develop a test method that would measure the transverse and longitudinal tensile properties of small diameter tubular polymers in their natural tubular form on Cal Poly’s campus.

Dr. Cardinal's vascular tissue engineering research group on Cal Poly’s campus is currently researching endothelialization of bare metal stents, in which ePTFE and PLGA are being used as scaffold materials for "blood vessel mimics" (BVM) [12]. These two materials are also commonly used in vascular graft applications, thus they were used to test the newly designed test fixtures [14, 19].

After several design iterations, a transverse test fixture was fabricated to meet the initial design requirements. The first of these requirements was a test fixture that was compatible with the Instron In-Spec 2200 testing machine. The initial design also 
required an appropriate fixture size that would fit the test sample and keep it as close to its original tubular shape as possible while pulling it apart in a uniaxial direction. Finally, the final test fixture needed a material that could withstand the force of a normal transverse tensile test for vascular graft materials (less than $50 \mathrm{~N}$ ). The final transverse fixture was successful in meeting these design criteria; however, the following section will point out specific challenges and limitations that were uncovered during the design process.

\subsection{Final Transverse Test Fixture Design Challenges and Limitations}

\subsubsection{Manufacturing}

The final design was fabricated in part on Cal Poly’s campus using the Mechanical Engineering department's Mustang '60 machine shop, while the rest of the piece was made by an off-campus machine shop due to limited capabilities of the Mustang '60 machine shop staff. Both machine shops were inexperienced in machining parts on such a small scale; thus, it was challenging to secure the rod of such small dimensions exactly perpendicular to the face it was protruding from. Also, the tack weld that secured the rod to the connection grip gave the slightest amount of deflection when force was applied to the rod. This deflection was not measured, and it would be hard to tell if this had any effect on the transverse tensile results.

\subsubsection{Compliance to Standards}

ISO 7198, which describes a test method for finding the transverse tensile strength in regards to tubular vascular prostheses, is very vague in describing a rod that 
should be "appropriately sized for the sample prosthesis to be held" [47]. The final transverse test fixture met this criteria with a rod conformed to the shape and size of the ePTFE and PLGA test samples; however, ISO 7198 does not offer much guidance on accurately measuring the transverse tensile properties of small diameter tubular polymers. In addition, no peer-reviewed literature could be found that cited ISO 7198 for testing vascular graft material or native vessels, which shows even more of a need for a standardized test procedure.

The final transverse test fixture was tested using the Instron In-Spec machine. The test samples remained in tubular form and were prepared similarly to methods described in literature and standards as explained in Chapter 4 [5, 10, 47, 51, 54, 58]. According to ASTM Standard D 2290 (V 08.04), to test the hoop tensile strength for thermoplastic piping of any size, the test sample should have a notch cut out with an inner width of 0.25 inches and a radius of 0.125 inches as cited in Chapter 1 [51]. In order to comply with this standard, the test sample tubes were cut using a drill press with a $1 / 4$ " diameter hollow hole as shown in Chapter 4 . While the radius was compliant with the ASTM Standard D 2290, it was not small enough to create a notch in the ePTFE or PLGA tubes that would localize the deformation in the test sample as it is diagrammed in ASTM Standard D 2290 and also shown in Figure 8. Also, due to material availability, the widths of the samples were thinner than 0.25 inches.

\subsection{Discussion of Statistical Analysis for Transverse Test Fixture}

The data collected during testing included ultimate tensile strength (UTS) and elastic modulus (E) of both ePTFE and PLGA. Seven samples of ePTFE and fourteen 
samples of PLGA were tested in tubular form then compared to six samples of ePTFE and seventeen samples of PLGA using the current method of testing. Results of the UTS tests showed a statistical difference between the new transverse test fixture versus the current method of testing for both ePTFE and PLGA. The average UTS for both ePTFE and PLGA was larger for the test samples kept in tubular form compared to those samples that were cut and laid flat. The average UTS for PLGA tubular samples 3.83 MPa compared to a 3.06 MPa average for the cut samples. For ePTFE, the average UTS of the tubular samples was 9.34 MPa compared to an average of $5.64 \mathrm{MPa}$ of the cut samples. There was no correlation between the sample's UTS and cross-sectional size for either test method or material.

\subsubsection{Stress-Strain Data for PLGA and ePTFE Testing}

The graphs below show the stress-strain curves for the PLGA samples tested in tubular form above the samples tested using the current method. A notable difference in the shape of the curves between Figure 58 and Figure 59 is the yield stress point. As explained in Chapter 1, the yield stress is the point at which plastic, or permanent, deformation begins to occur [8]. In Figure 58, the stress-strain curve of PLGA tested in tubular form has more of a defined yield stress point followed by a gradual plastic deformation region. Figure 59, on the other hand, has a less defined yield stress point and more of a sweeping arc between the elastic and plastic deformation regions for material tested using the current method. Because PLGA has so many different makeups and applications, there is no well documented stress-strain behavior for this material, making it difficult to analyze the difference between these two stress-strain curves. 


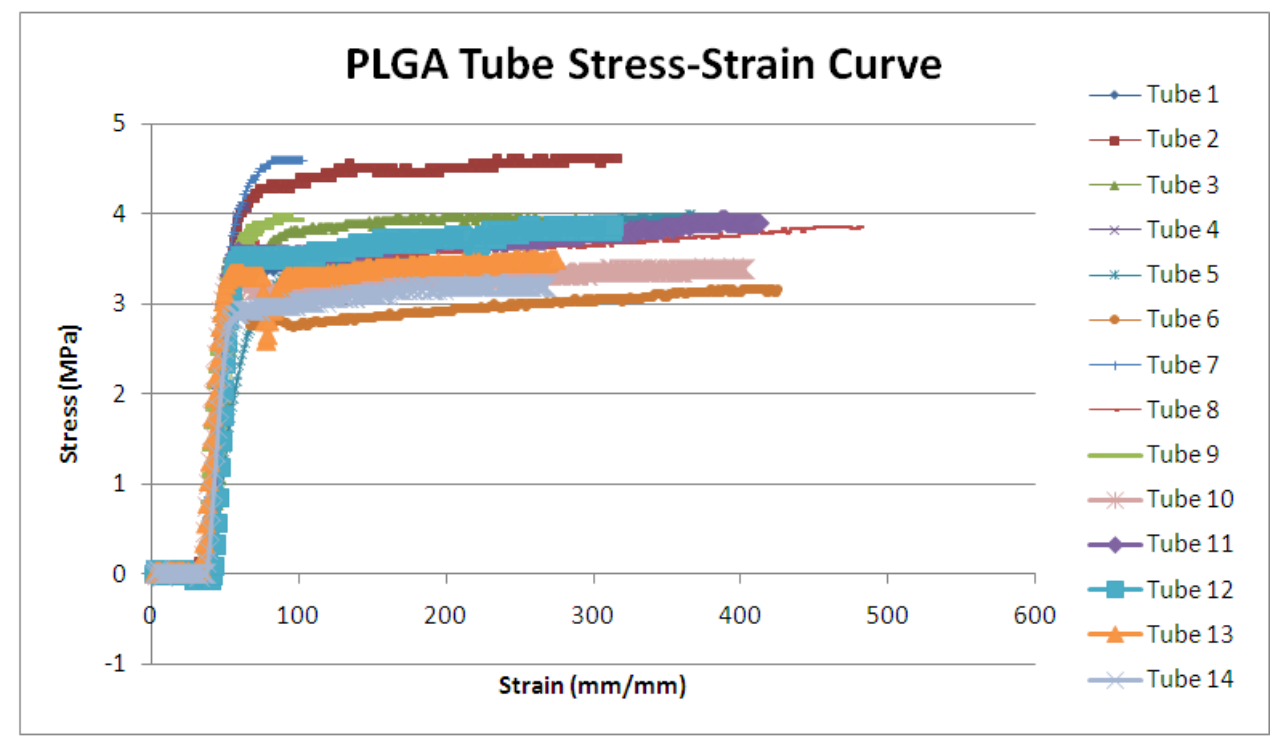

Figure 58: PLGA stress-strain curve for samples tested in tubular form using the transverse test fixture.

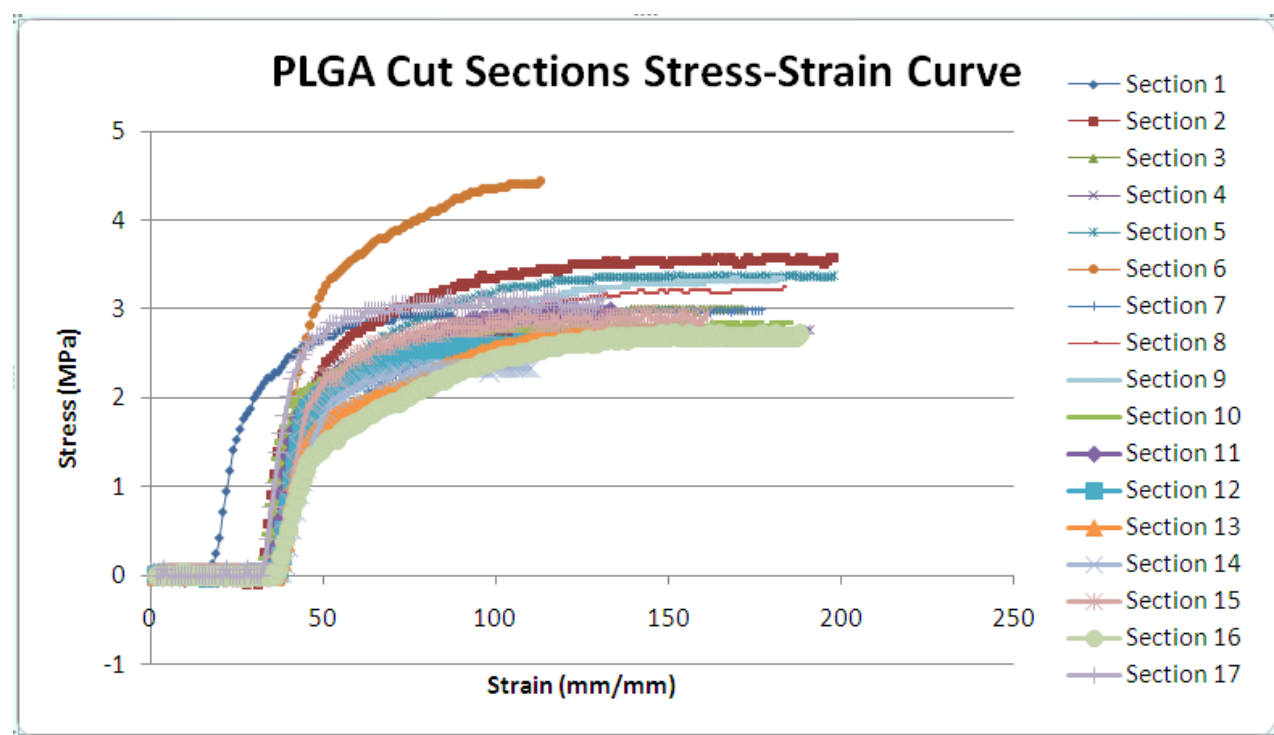

Figure 59: PLGA stress-strain curve for samples tested using the current method of cutting the sample and laying it flat between the Instron grips.

The stress-strain curves of the ePTFE test samples using the two different test methods have a more similar shape to each other than the PLGA curves, even though the 
UTS between test methods was significantly different. Both of these graphs are shown below.

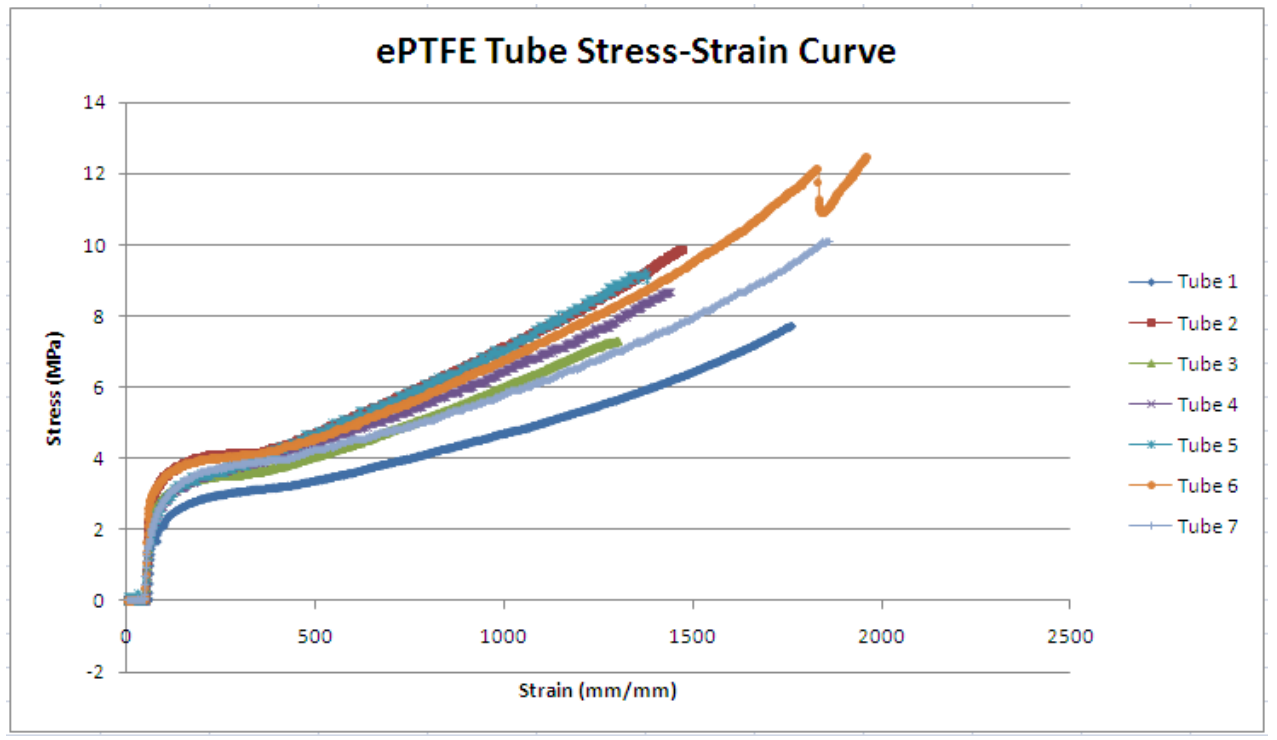

Figure 60: ePTFE stress-strain curve for samples tested in tubular form using the new transverse test fixture.

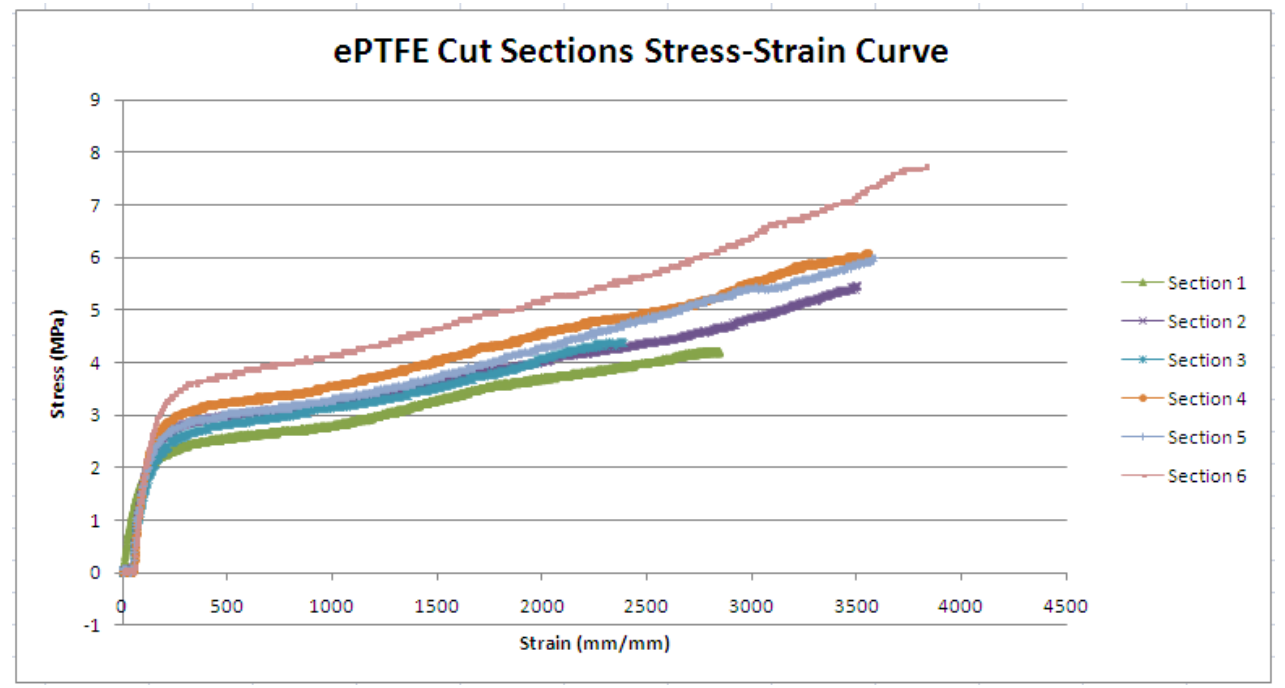

Figure 61: ePTFE stress-strain curve for samples tested using the current method of cutting the sample and laying it flat between the Instron grips. 
Another important difference between test methods that was observed during testing was the location on each test sample where the fracture occurred. For both ePTFE and PLGA samples tested using the current method of cutting the sample and laying it flat, fracture tended to occur on the piece of material closest to the Instron machine grips. As noted in Chapter 4, there were two test samples of ePTFE that slipped from the grips of the Instron, thus the data for those tests were discounted. All test samples that were tested in tubular form had deformation and fracture between the two connection grips and within the notch of the sample; however, fracture usually occurred at only one point on the tubular sample. Below shows a picture of the fractured samples of ePTFE tested in tubular form compared to being cut and laid flat.

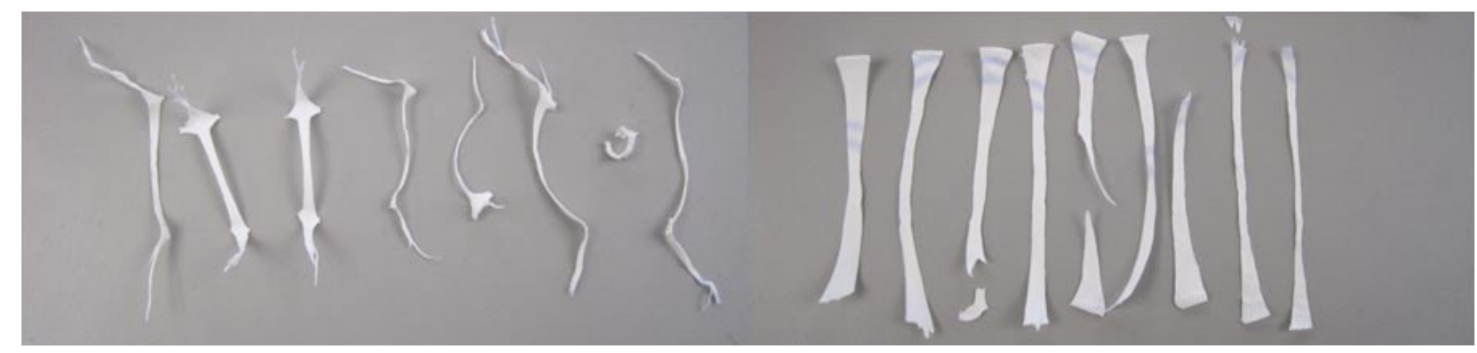

Figure 62: The ePTFE samples shown to the left were tested in tubular form while the samples on the right were tested after the sample was cut and laid flat between the Instron grips. Note the samples tested in tubular form fractured on only one side of the tube, but fractured in the middle of the notch. The samples that were cut fractured closer to where they were attached to the Instron grips.

While the experimental study based on UTS showed consistent results between ePTFE and PLGA, it is difficult to measure which test method is more accurate. Comparing the results with already documented transverse UTS measurements of both 
ePTFE and PLGA would be suggested; however, as discussed in Chapter 1, there are not many documented transverse measurements to be found. Also, many of these documented transverse measurements use the method of cutting the sample and laying it flat $[3,32,37]$. Furthermore, ePTFE has varying internodal distances, and PLGA has different weights of lactide versus glycolide acid $[19,40]$. These variations provide an additional obstacle in uncovering the material's true transverse tensile properties, as well as the correct method of testing for these properties.

Statistical results for the elastic modulus data did not offer as clear cut conclusions as did the UTS data. There was no significant difference between test methods based on the ePTFE data, and no conclusions could be drawn from the elastic modulus data collected from the PLGA samples. For the elastic modulus data collected for ePTFE, it is interesting to note that there was one outlier in the samples tested in tubular form. Tube number seven had an elastic modulus of 1.94 MPa, but had a fairly normal UTS compared to the rest of the ePTFE samples tested in tubular form. Tube number seven's cross-sectional area was also common among the other test samples. Also, the ePTFE pilot study outlined in Chapter 4 required fifty-four samples to be tested in order to ensure a $95 \%$ level of confidence. Due to material availability and a few unsuccessful tests as mentioned earlier, only a fraction of the ideal sample size could be reached. Thus, it is difficult to draw true conclusions regarding the effect on UTS or elastic modulus due to test method for ePTFE.

It was interesting to see such a large difference in variability among the UTS and elastic modulus data for PLGA. While the UTS data met all of the requirements to perform an ANOVA study, the elastic modulus results did not show equal variances. In 
fact, the standard deviation for the current method of testing data was seven times greater than the standard deviation for the tubular test samples. The average elastic modulus for the tubular testing method was 7.69 MPa compared to $39.33 \mathrm{MPa}$ for the current method of testing. Unfortunately, there is not a lot of other documented elastic modulus data collected for the transverse direction of PLGA (85/15). Pena cited a $31.48 \mathrm{MPa}$ transverse elastic modulus for PLGA that was fabricated on the same machine at Cal Poly, and tested using the current method of cutting the sample and laying it flat [12]. That shows consistency compared to our results for the current method, but does not grant validity to the newly designed transverse test fixture. Based on the previously discussed design challenges and limitations for the transverse test fixture, the next section

will suggest future work to resolve these issues and expand on the design and testing that has already been done.

\subsection{Future Work}

Based on ASTM and ISO standards, as well as documented test methods from literature, there seems to be no single, specific method to date for testing the tensile properties of small diameter tubular polymers. The goal of this thesis was to incorporate elements from these standards and literature to create a method of transverse and longitudinal tensile testing that would not only benefit Cal Poly's campus, but create awareness for the need of a more universal way of testing specifically for vascular graft materials, native vessels, and vascular tissue engineered constructs. That being said, there is still work to be done to provide enough evidence to suggest a standard, accurate method of transverse testing. This section will point out some suggestions that would 
help expand and improve upon the work of this thesis, as well as suggest future experiments that could lead to a more standardized method of testing.

\subsubsection{Randomization and More Extensive Testing}

The biggest limitation of this thesis was the lack of randomization in the experimental study. Both of the ePTFE and PLGA samples tested were fabricated from the same few lengths of tubing provided at the time of study. Ideally, each test sample would come from different lengths of tubing fabricated using a consistent method at different times. This would allow assumptions to be made for the entire population of that specific material, instead of only the lengths of tubes provided. Additionally, the lack of available ePTFE tubing made it difficult to conclude a significant difference in test methods. No conclusion could be drawn from the PLGA testing due to unequal variances; however, with a larger sample size, that variance could be reduced. Thus, more extensive testing of both ePTFE and PLGA could better determine the significance of using the both the longitudinal and transverse test fixtures.

It was also hard to compare transverse tensile test methods due to lack of currently documented transverse tensile properties of ePTFE and PLGA. ePTFE and PLGA are materials that have varying make-ups as mentioned previously, such as internodal distance (ePTFE) and mole fractions (PLGA) [19, 40]. These materials were chosen because of their availability on campus and their use by Cal Poly's tissue engineering group. It would be beneficial to test a more consistent or simpler material that has been extensively tested and researched for further testing of the new transverse fixture so it could be more easily compared to already documented tensile strengths. 
Silicone, polyurethane, and polypropylene tubing are all commonly used and fairly non-variable materials that have a broad range of applications and also come in small diameter dimensions. Because a number or companies offer these products in identical formulas and sizes, such as NewAge Industries, Grainger, and Hudson Extrusions, Inc., each material could be tested and analyzed randomly. Most of these companies only offered burst pressure as a tensile strength measurement on their websites, thus further research is needed to determine an average ultimate tensile strength (MPa) and elastic modulus (MPa) for these materials [59-61].

\subsubsection{More Detailed Observations During Testing}

Another limitation to this experimental study occurred during testing. While each test was observed and oddities were noted, for example, if the test sample slipped out of the connection grips, there were not specific observations made and documented as to the behavior of each sample during testing. There were a number of outliers recorded for both UTS and elastic modulus in both materials that did not correlate to the crosssectional size of the test sample. This could be due to the sample deforming differently between the connection grips or being placed in a different way onto the rods of the test fixture. Great care was taken to make sure all test samples were tested consistently; however, more specific testing observations would be beneficial to analyze the results. Even video recording each test in the future would be recommended so that some correlation or explanation could be suggested for outlying results. This suggestion would apply to testing with both the transverse and longitudinal test fixtures, especially due to the high shear stress created in the longitudinal fixture and risk of the sample tearing at the grips. 


\subsubsection{Burst Pressure Testing}

As discussed in Chapter 1, about half of the literature reviewed for this thesis used burst pressure as a method of characterizing the mechanical properties of vascular grafts. This could be another suitable option for testing the properties of small diameter tubes on Cal Poly's campus, but as mentioned previously, Cal Poly currently does not have the capabilities of burst testing these types of tubes. Furthermore, burst pressure testing measures the maximal internal pressure at which a tubular construct breaks [44]. This is similar to the way ultimate tensile strength characterizes a material, only it is usually measured in pounds per square inch (psi) instead of MPa.

The fallback of burst pressure, however, is it does not measure the elasticity of the tubular construct. Elasticity is an important property to know for vascular graft materials due to intimal hyperplasia, the most common cause of long time failure in small diameter vascular grafts $[2,17]$. Different elasticities between blood vessels and vascular grafts lead to differences in shear stress at the endothelium. This injury then leads to intimal hyperplasia [2,34]. In order to reduce the occurrence of this problem, vascular grafts must maintain matching, or as close to matching tensile properties as possible. This explains the need for accurate methods of testing the transverse elastic modulus of vascular graft materials. While this thesis did not come to any valid conclusions regarding the elastic modulus results of ePTFE and PLGA, further testing, as mentioned above, is recommended in order to continue the search for a universally accepted, accurate method of testing the transverse, as well as longitudinal properties of small diameter tubular polymers. 


\subsection{Conclusion}

In order to create an optimal replacement for vessels diseased from coronary and peripheral artery disease, the replacement, or vascular graft, must mimic the characteristics of a native, healthy vessel. Among these characteristics are the mechanical properties, including the ultimate tensile strength and elastic modulus that are tested in both the longitudinal and transverse directions. Today, there is no "gold standard" for testing these properties for small diameter tubes in the transverse direction. While efforts have been made to design a method of testing that accurately measures these properties, more studies must be conducted and literature produced so as to provide a comparison for other researchers' methods and results. Once an appropriate method of testing has been collectively accepted, it will open new doors to finding the optimal vessel replacement material. The work described in this thesis will provide the foundation for further research on tensile test methods for small diameter tubular polymers, and encourage future work in this area on Cal Poly’s campus. 


\section{List of References}

1. Ravi, S., Z. Qu, and E.L. Chaikof, Polymeric materials for tissue engineering of arterial substitutes. Vascular, 2009. 17 Suppl 1: p. S45-54.

2. Greenwald, S.E. and C.L. Berry, Improving vascular grafts: the importance of mechanical and haemodynamic properties. J Pathol, 2000. 190(3): p. 292-9.

3. Iwasaki, K., et al., Bioengineered three-layered robust and elastic artery using hemodynamically-equivalent pulsatile bioreactor. Circulation, 2008. 118(14 Suppl): p. S52-7.

4. Kannan, R.Y., et al., Current status of prosthetic bypass grafts: a review. J Biomed Mater Res B Appl Biomater, 2005. 74(1): p. 570-81.

5. Berglund, J.D., R.M. Nerem, and A. Sambanis, Viscoelastic testing methodologies for tissue engineered blood vessels. J Biomech Eng, 2005. 127(7): p. 1176-84.

6. Craig, R.R., ed. Mechanics of Materials. 1996, John Wiley \& Sons: New York.

7. Dowling, N.E., ed. Mechanical Behavior of Materials. 1993, Prentice Hall: Englewood Cliffs, NJ.

8. Smith, W.F., ed. Principles of Materials Science and Engineering. 3 ed. 1996, McGraw-Hill, Inc.: New York.

9. Roh, J.D., et al., Small-diameter biodegradable scaffolds for functional vascular tissue engineering in the mouse model. Biomaterials, 2008. 29(10): p. 1454-63.

10. Soffer, L., et al., Silk-based electrospun tubular scaffolds for tissue-engineered vascular grafts. J Biomater Sci Polym Ed, 2008. 19(5): p. 653-64. 
11. Thomas, V., et al., Functionally graded electrospun scaffolds with tunable mechanical properties for vascular tissue regeneration. Biomed Mater, 2007. 2(4): p. 224-32.

12. Pena, T.R., Preparation and Characterization of Electrospun Poly(D,L-LactideCo-Glycolide) Scaffolds for Vascular Tissue Engineering and the Advancement of an In Vitro Blood Vessel Mimic, in Biomedical Engineering. 2009, California Polytechnic State University: San Luis Obispo.

13. Baguneid, M.S., et al., Tissue engineering of blood vessels. Br J Surg, 2006. 93(3): p. 282-90.

14. Ravi, S. and E.L. Chaikof, Biomaterials for vascular tissue engineering. Regen Med. 5(1): p. 107-20.

15. Wikol, M., et al., Expanded Polytetrafluoroethylene Membranes and Their Applications. Filtration in the Biopharmaceutical Industry, 1998: p. 619-640.

16. Xue, L. and H.P. Greisler, Biomaterials in the development and future of vascular grafts. J Vasc Surg, 2003. 37(2): p. 472-80.

17. Wu, L., et al., "Wet-state" mechanical properties of three-dimensional polyester porous scaffolds. J Biomed Mater Res A, 2006. 76(2): p. 264-71.

18. Jeong, S.I., et al., Tissue-engineered vascular grafts composed of marine collagen and PLGA fibers using pulsatile perfusion bioreactors. Biomaterials, 2007. 28(6): p. 1115-22.

19. Leung, L., et al., Comparison of morphology and mechanical properties of PLGA bioscaffolds. Biomed Mater, 2008. 3(2): p. 025006. 
20. Doshi, J. and D.H. Reneker, Electrospinning Process and Applications of Electrospun Fibers. Journal of Electrostatics, 1995. 35: p. 151-160.

21. Liatsikos, E., et al., Ureteral stents: past, present and future. Expert Rev Med Devices, 2009. 6(3): p. 313-24.

22. Ates, Y., et al., The mechanical properties of intact and traumatized epidural catheters. Anesth Analg, 2000. 90(2): p. 393-9.

23. Tanaka, S., et al., Mechanical Properties of the Bioabsorbable Polyglycolic AcidCollagen Nerve Guide Tube. Polymer Engineering and Science, 2006: p. 14611467.

24. Deusch, E., et al., The mechanical properties of continuous spinal small-bore catheters. Anesth Analg, 2004. 99(6): p. 1844-7, table of contents.

25. Bell, A.L., R. Jayaraman, and L.M. Vercaigne, Effect of ethanol/trisodium citrate lock on the mechanical properties of carbothane hemodialysis catheters. Clin Nephrol, 2006. 65(5): p. 342-8.

26. Tsui, B.C. and B. Finucane, Tensile strength of 19- and 20-gauge arrow epidural catheters. Anesth Analg, 2003. 97(5): p. 1524-6.

27. Plikk, P., S. Malberg, and A.C. Albertsson, Design of resorbable porous tubular copolyester scaffolds for use in nerve regeneration. Biomacromolecules, 2009. 10(5): p. 1259-64.

28. Lange, D., et al., Uropathogen interaction with the surface of urological stents using different surface properties. J Urol, 2009. 182(3): p. 1194-200. 
29. Ramesh, P., R. Joseph, and M.C. Sunny, A comparative evaluation of coefficient of friction and mechanical properties of commercially available Foley catheters. $\mathrm{J}$ Biomater Appl, 2001. 15(4): p. 344-50.

30. American Heart Association Heart Disease and Stroke Statistics-2009 Update. 2009, American Heart Association: Dallas, Texas.

31. Kurane, A., D.T. Simionescu, and N.R. Vyavahare, In vivo cellular repopulation of tubular elastin scaffolds mediated by basic fibroblast growth factor. Biomaterials, 2007. 28(18): p. 2830-8.

32. Teng, Z., et al., An experimental study on the ultimate strength of the adventitia and media of human atherosclerotic carotid arteries in circumferential and axial directions. J Biomech, 2009. 42(15): p. 2535-9.

33. Sell, S.A., et al., Electrospun polydioxanone-elastin blends: potential for bioresorbable vascular grafts. Biomed Mater, 2006. 1(2): p. 72-80.

34. Salacinski, H.J., et al., The mechanical behavior of vascular grafts: a review. J Biomater Appl, 2001. 15(3): p. 241-78.

35. Tiwari, A., et al., New prostheses for use in bypass grafts with special emphasis on polyurethanes. Cardiovasc Surg, 2002. 10(3): p. 191-7.

36. Shimada, T., et al., Improved healing of small-caliber, long-fibril expanded polytetrafluoroethylene vascular grafts by covalent bonding of fibronectin. Surg Today, 2004. 34(12): p. 1025-30.

37. Isaka, M., et al., Experimental study on stability of a high-porosity expanded polytetrafluoroethylene graft in dogs. Ann Thorac Cardiovasc Surg, 2006. 12(1): p. 37-41. 
38. Greene, D., L. Pruitt, and C.S. Maas, Biomechanical effects of e-PTFE implant structure on soft tissue implantation stability: a study in the porcine model. Laryngoscope, 1997. 107(7): p. 957-62.

39. Doble, M., et al., Analysis of explanted ePTFE cardiovascular grafts (modified BT shunt). Biomed Mater, 2008. 3(3): p. 034118.

40. Catanese, J., 3rd, et al., Mechanical properties of medical grade expanded polytetrafluoroethylene: the effects of internodal distance, density, and displacement rate. J Biomed Mater Res, 1999. 48(2): p. 187-92.

41. Crapo, P.M. and Y. Wang, Physiologic compliance in engineered small-diameter arterial constructs based on an elastomeric substrate. Biomaterials. 31(7): p. 1626-35.

42. Li, P., et al., Influences of tensile load on in vitro degradation of an electrospun poly(l-lactide-co-glycolide) scaffold. Acta Biomater.

43. Shearer, H., et al., Effects of common sterilization methods on the structure and properties of poly(D,L lactic-co-glycolic acid) scaffolds. Tissue Eng, 2006. 12(10): p. 2717-27.

44. Arsene, S. and J. Bai, A New Approach to Measuring Transverse Properties of Structural Tubing by a Ring Test. Journal of Testing and Evaluation, 1996. 24(6): p. 386-391.

45. Instron Worldwide. [cited 2010; Available from: http://www.instron.us/.

46. Standard Test Method for Tensile Properties of Plastics. 2007, ASTM International. 
47. Cardiovascular implants - Tubular vascular prostheses. 1998, International Organization for Standardization.

48. Hinds, M.T., et al., Development of a reinforced porcine elastin composite vascular scaffold. J Biomed Mater Res A, 2006. 77(3): p. 458-69.

49. Zhu, C., et al., Initial investigation of novel human-like collagen/chitosan scaffold for vascular tissue engineering. J Biomed Mater Res A, 2009. 89(3): p. 829-40.

50. Guan, J., et al., Preparation and characterization of highly porous, biodegradable polyurethane scaffolds for soft tissue applications. Biomaterials, 2005. 26(18): $\mathrm{p}$. 3961-71.

51. Standard Test Method for Apparent Hoop Tensile Strength of Plastic or Reinforced Plastic Pipe by Split Disk Method. 2007, ASTM International.

52. Krashchenko, V.P. and A.I. Gurarii, Methods of Tensile Testing Ring Specimens (Review). Zavodskaya Laboratoriya, 1985. 51(11): p. 66-69.

53. Wang, H., et al., Ring Hoop Tension Test (RHTT): A Test for Transverse Tensile Properties of Tubular Materials. Journal of Testing and Evaluation, 2002. 30(5).

54. Seliktar, D., et al., Dynamic mechanical conditioning of collagen-gel blood vessel constructs induces remodeling in vitro. Ann Biomed Eng, 2000. 28(4): p. 351-62.

55. Madison, J., CNC Machining Handbook: Basic Theory, Production Data, and Machining Procedures. 1996, New York, NY: Industrial Press Inc.

56. Jindal, U.C., Machine Design. 2010: Pearson Education India. 892.

57. Ott, R.L. and M. Longnecker, An Introduction to Statistical Methods and Data Analysis. Sixth ed. 2010: CENGAGE Learning. 
58. Crapo, P.M., J. Gao, and Y. Wang, Seamless tubular poly(glycerol sebacate) scaffolds: high-yield fabrication and potential applications. J Biomed Mater Res A, 2008. 86(2): p. 354-63.

59. NewAge Industries. 2011; Available from: http://www.newageindustries.com/.

60. W. W. Grainger, Inc. 2011; Available from: http://www.grainger.com/Grainger/wwg/start.shtml.

61. Hudson Extrusions Inc. 2011; Available from: http://www.hudsonextrusions.com/. 


\section{Appendix A: ASTM D 2290-Standard Test Method for}

\section{Apparent Hoop Tensile Strength of Plastic or Reinforced}

\section{Plastic Pipe by Split Disk Method}

Standard Test Method for Apparent Hoop Tensile Strength of Plastic or Reinforced Plastic Pipe by Split Disk Method ${ }^{1}$

This standard is issued under the fixed designation D 2290, the number immediately following the designation indicates the year of original adoption or, in the case of revision, the year of last revision. A number in parentheses indicates the year of last reapproval, A superscript cpsilon $(e)$ indicates an editorial change since the last revision or reapproval.

This standant has been approved for we by agencies of the Deparmment of Defense.

\section{Scope}

1.1 This test method covers the determination of the comparative apparent tensile strength of most plastic products utilizing a split disk test fixture, when tested under defined conditions of pretreatment, temperature, humidity, and test machine speed. This test method is applicable to reinforcedthermosetting resin pipe regardless of fabrication method. This test method also is applicable to extruded and molded thermoplastic pipe.

Procedure $\mathrm{A}$ is used for reinforced-thermosetting resin pipe: Procedure B is used for thermoplastic pipe of any size: Procedure $\mathrm{C}$ is used for thermoplastic pipe with nominal diameter of $4 \frac{1}{2} \mathrm{in}$. and greater.

1.2 The values stated in inch-pound units are to be regarded as standard. The values given in parentheses are mathematical conversions to $\mathrm{SI}$ units that are provided for information only and are not considered standard.

13 This standard does not purport to address all of the safery concerns, if any, associated with its use. It is the responsibility of the user of this standard to establish appropriate safety and health practices and determine the applica. bility of regulatory limitations prior to use.

2. Referenced Documents

2.1 ASTM Standands:2

D 618 Practice for Conditioning Plastics for Testing

D 1599 Test Method for Resistance to Short-Time Hydrau-

lie Pressure of Plastic Pipe, Tubing, and Fittings

E 4 Practices for Force Verification of Testing Machines

3. Summary of Test Method

3.1 The test specimen is loaded through the suggested self-aligning split disk test fixture (Fig. 1) which applies tensile

This test method is under the jurisdiction of ASTM Committee F17 on Plastic Piping Systems and is the dires responsibility of Subcomminter F17.40 on Test Piping Syuc

Current edition approved Nov, 1, 2008. Published November 2008 . Originally approved in 1964 . Last previous edition approved in 2004 as D $2290-04$.

${ }^{2}$ For referenced ASTM standards, visit the ASTM wetsite, www wastm. org, of eontact ASTM Customer Service at service te antm. org. For Annual Book of ASTM Standact ASTM Customer Service at service Gastmi. org. For Annias Boek of ASTM the ASTM wetwite. stress to the test ring. An apparent tensile strength rather that a true tensile strength is obtained in this test because of a bending moment imposed during test at the split between the split disk test fixture. This moment is induced by the change is contour of the ring between the two disk sections as they separate. The test fixture is designed to minimize the effect of this bending moment.

4. Significance and Use

4.1 Split disk tension tests, properly interpreted, provid reasonably accurate information with regard to the apparea tensile strength of plastic pipe when employed under condi tions approximating those under which the tests are made.

4.2 Tension tests may provide data for research and devel. opment, engineering design, quality control, acceptance of rejection under specifications, and for special purposes. The test cannot be considered significant for applications differing widely from the load-time scale of the standard test.

Nom: 1-Procedure $\mathrm{C}$ has been used on polyethylene and polybutylene pipe to produce results equivalent to Quick Burst results (Test Method D 1599) for 4 in. to 8 in. pipes

\section{Apparatus}

5.1 Micrometers-Suitable ball-type micrometers, reading to at least $0.001 \mathrm{in}$., for measuring the width and thickness of the test specimens.

5.2 Testing Machine-A universal testing machine of the constant-rate-of-cross-head-movement type and comprising essentially the following:

5.2.1 Drive Mechanism-A drive mechanism for imparting to the crosshead a uniform controlled velocity with respect to the base, this velocity to be regulated as specified in Section 9

5.2.2 Load Indicator-A load-indicating mechanism car pable of showing the total tensile load carried by the tes specimen. This mechanism shall be essentially free from inertia lag at the specified rate of testing and shall indicate the load with an accuracy of $\pm 1 \%$ of the indicated value. The accuracy of the testing machine shall be verified in accordance with Method E 4.

5.3 Test Fixture- The recommended test fixture is shown it Fig. 1. The width of the split disk for Procedure $\mathrm{A}$ and Procedure B shall be at least 0.1 in. greater than the width of 


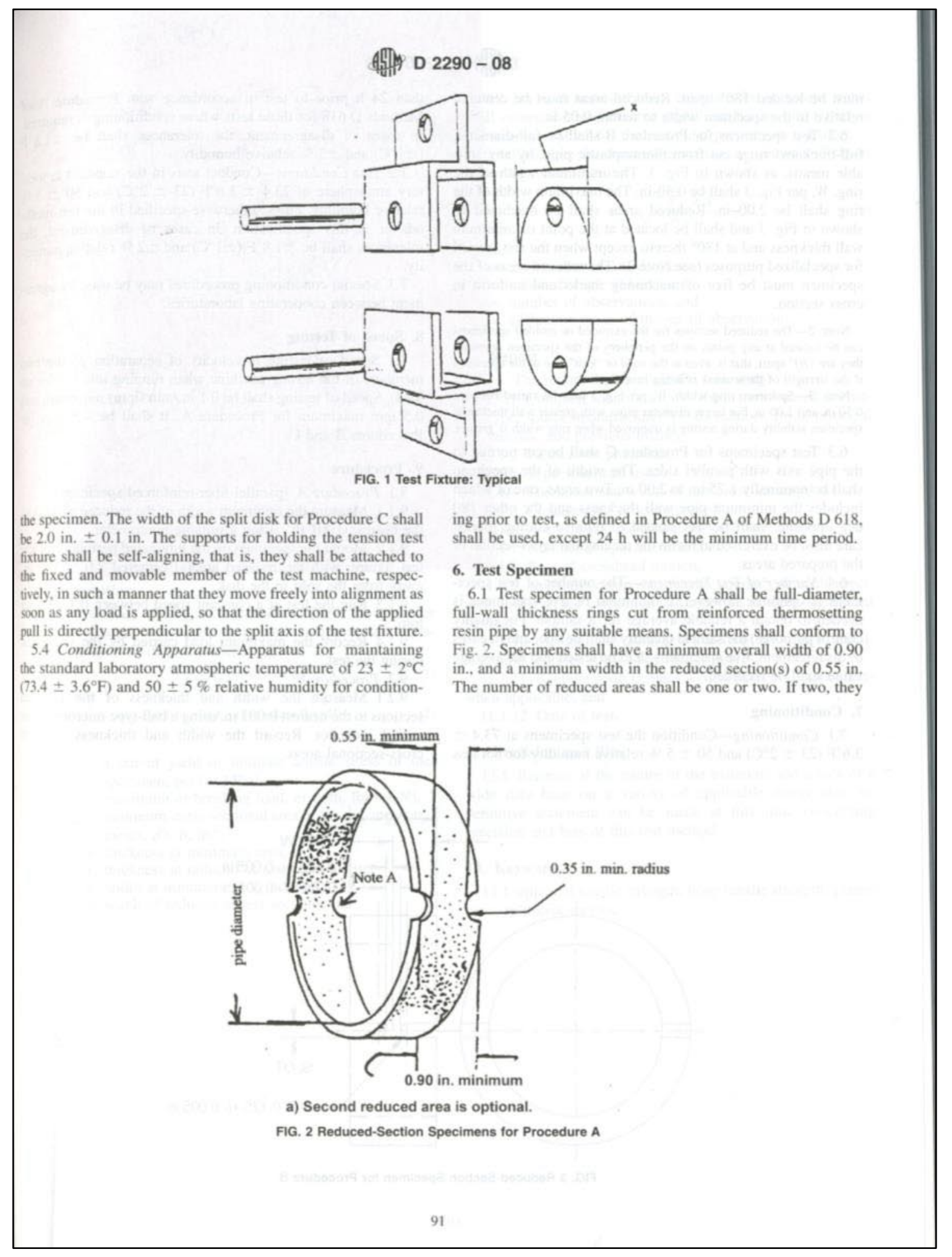




\section{2290-08}

must be located $180^{\circ}$ apart. Reduced areas must be centered relative to the specimen width to within 0.05 in.

6.2 Test specimens for Procedure B shall be full-diameter, full-thickness rings cut from thermoplastic pipe, by any suitable means, as shown in Fig. 3. The minimum width of the ring, $W$, per Fig. 3 shall be 0.50 -in. The maximum width of the ring shall be 2.00 -in. Reduced areas shall be machined as shown in Fig. 3 and shall be located at the point of minimum wall thickness and at $180^{\circ}$ thereto except when the test is used for specialized purposes (see Note 2). The reduced areas of the specimen must be free of machining marks and uniform in cross section.

Norn 2-The reduced sections for the extruded or molded specimens can be located at any points on the periphery of the specimen provided they are $180^{\circ}$ apart, that is, even at the weld or "knit" line of the specimen if the strength of these areas is being investigated.

Nort 3-Specimen ring width, $W$, per Fig. 3 may be varied between 0.50 in. and $2.00 \mathrm{in}$. For larger diameter pipes with greater wall thickness, specimen stability during testing is improved when ring width is greater.

6.3 Test specimens for Procedure $\mathrm{C}$ shall be cut normal to the pipe axis with parallel sides. The width of the specimen shall be nominally 1.75 in. to 2.00 in. Two areas, one of which includes the minimum pipe wall thickness and the other 180 deg opposite, shall be wet sanded to remove cutting marks; care must be exercised to retain the rectangular cross-section of the prepared areas.

6.4 Number of Test Specimens-The number of test specimens is optional; however, a minimum of five specimens is needed to obtain a reliable average for a sample. For quality control purposes, the sample quantity shall be one, and in cases of disagreement, five specimens shall be tested and the average value shall be recorded.

\section{Conditioning}

7.1 Conditioning - Condition the test specimens at $73.4 \pm$ $3.6^{\circ} \mathrm{F}\left(23 \pm 2^{\circ} \mathrm{C}\right)$ and $50 \pm 5 \%$ relative humidity for not less than $24 \mathrm{~h}$ prior to test in accordance with Procedure A dit Methods D 618 for those tests where conditioning is required In cases of disagreement, the tolerances shall be $\pm 1.8^{\circ}$ $\left( \pm 1^{\circ} \mathrm{C}\right)$ and $\pm 2 \%$ relative humidity.

7.2 Test Conditions - Conduct tests in the standard labon tory atmosphere of $73.4 \pm 3.6^{\circ} \mathrm{F}\left(23 \pm 2^{\circ} \mathrm{C}\right)$ and $50 \pm 5$ s relative humidity, unless otherwise specified in the test mett ods or in this specification. In cases of disagreement, the tolerances shall be $\pm 1.8^{\circ} \mathrm{F}\left( \pm 1^{\circ} \mathrm{C}\right)$ and $\pm 2 \%$ relative humid. ity.

7.3 Special conditioning procedures may be used by agree ment between cooperating laboratories.

\section{Speed of Testing}

8.1 Speed of testing is velocity of separation of the twe members of the testing machine when running idle (under no load). Speed of testing shall be $0.1 \mathrm{in} / \mathrm{min}$ (ipm) minimum an 0.5 ipm maximum for Procedure A. It shall be 0.5 ipm fo Procedures B and C.

\section{Procedure}

9.1 Procedure A (parallel-fiber-reinforced specimens):

9.1.1 Measure the minimum width of the reduced area(s) the nearest 0.001 in., using a suitable micrometer.

9.1.2 Mount the specimen on the lubricated periphery of th: test fixture, with the reduced area(s) centered $2.0 \pm 0.2$ i away from the split in the disk.

9.1.3 Run the test at a constant speed between 0.1 and 0.5 ipm.

9.1.4 Record the maximum load carried by the specime during the test.

9.2 Procedure $B$ :

9.2.1 Measure the width and thickness of the reducel sections to the nearest 0.001 in. using a ball-type micrometer a dial indicator. Record the width and thickness of bot cross-sectional areas.

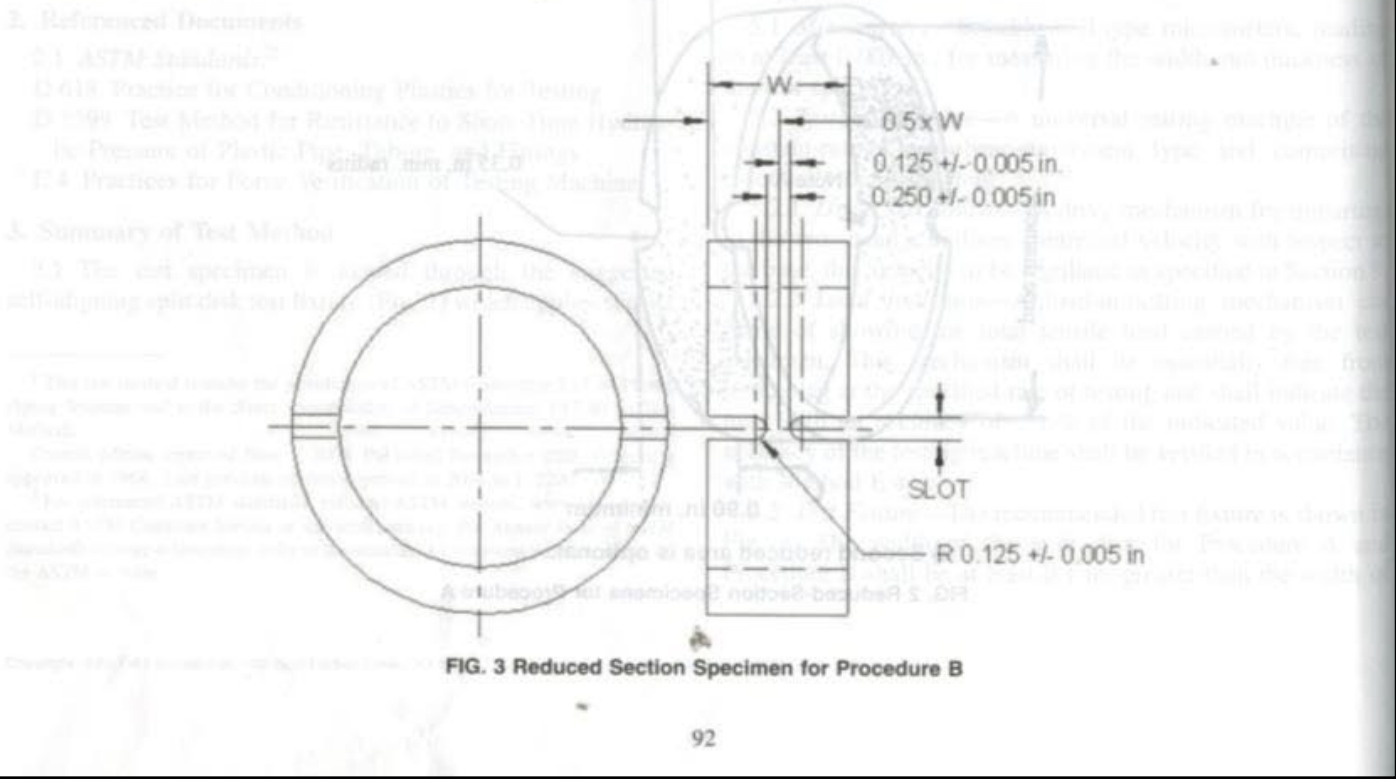




\section{Aflly D 2290-08}

9.2.2 Mount the test specimen on the split disk test fixture 9.2. Me reduced sections at the split in the fixture. Align the west specimen on the split disk specimen holder so that it is estered on the line joining the points of attachment of the fixture to the test machine.

9.2 .3 Set the speed control at $0.50 \mathrm{in} / \mathrm{min}$ and start the test machine.

92.4 Record the yield and ultimate loads carried by the specimen during the test.

9.3 Procedure C:

9.3.1 Measure the width and thickness of the prepared areas to the nearest 0.001 in. using a ball anvil type micrometer. Record the width and thickness of both prepared crosssectional areas.

9.3.2 Mount the test specimen on the outside of the split disk fixture with the prepared and measured areas located at the split in the fixture. Align the test specimen on the test fixture so that it is centered on the line joining the points of attachment to the test machine.

93.3 Set the speed control at $0.50 \mathrm{in} . / \mathrm{min}$ and start the machine.

9.3.4 Record the yield point and maximum load carried by the specimen during the test.

9.3.5 Record the crosshead separation required to rupture the test specimen.

\section{Calculation}

10.1 Calculate the apparent tensile strength (at yield or rupture, or both) of the specimen using the appropriate equation and report to three significant figures.

$$
\begin{gathered}
\sigma_{s}=P_{y} / 2 A_{m} \text { (Procedure A) } \\
\text { or: } P_{p} /\left(d_{1} b_{1}+d b_{2}\right) \text { (Procedure B or C) }
\end{gathered}
$$

where:

$\sigma_{0} \quad=$ apparent yield or ultimate tensile stress of the specimen, psi (or MPa),

$P_{h} \quad=$ maximum or breaking load, or both, Ibf (or N),

$A_{m}=$ minimum cross-sectional area of the two measurements, $d \times b$, in? ${ }^{2}$.

$d \quad=$ thickness at minimum area, in..

$d_{y_{1}} d_{2}=$ thickness at reduced or test sections, in

$b \quad=$ width at minimum area, in., and

$b_{1}, b_{2}=$ width of reduced or test sections, in.

10.2 For each series of tests, ealculate the arithmetic mean of all values obtained to three significant figures and report as the" average value" for the particular property in question.

10.3 Calculate the standard deviation as follows and report to two significant figures:

$$
s=\sqrt{\frac{\sum X-n \bar{X}^{2}}{n-1}}
$$

where:

$s=$ estimated standard deviation,

$X=$ value of single observation.

$n=$ number of observations, and

$\bar{X}=$ arithmetic mean of the set of observations.

\section{Report}

11.1 The report shall include the following:

11.1.1 Complete identification of the material tested, including type, source, manufacturer's code number, form, principal dimensions, and previous history.

11.1.2 Fabrication procedure,

11.1.3 Type specimen used,

11.1.4 Thickness and width of test cross-sectional area.

11.1.5 Conditioning procedure used,

11.6 Atmospheric conditions in test room.

11.1.7 Number of specimens tested,

11.1.8 Rate of crosshead motion.

11.1.9 Apparent composite tensile strength of each specimen, and average calculated and reported to three significant figures,

11.1.10 Standard deviation (estimated) of the sample calculated and reported to two significant figures.

11.1.11 Average resin content weight percent of specimens when applicable, and

11.1.12 Date of test.

\section{Precision and Bias}

12.1 Because of the nature of the materials and a lack of a wide data base on a variety of applicable composites, no definitive statement can be made at this time concerning precision and bias of this test method.

\section{Keywords}

13.1 apparent tensile strength; hoop tensile strength: plastic pipe; split-disk method 


\section{Appendix B: ISO 7198-Determination of Circumferential}

\section{Tensile Strength}

ISO 7198:1998(E)

Additional information shall be recorded in accordance with 4.9.2.

8.2.4 Determination of water entry pressure $(\mathrm{N})$

8.2.4.1 Principle

This test is intended to determine the water entry pressure of vascular prostheses.

\subsubsection{Apparatus}

Apparatus to be used include a machine capable of incrementally pressurizing samples until leakage occurs. An appropriate pressure transducer should also be used.

8.2.4.3 Sampling

Sampling shall be in accordance with clause 7.

8.2.4.4 Test procedure

Samples are filled with water and pressurized to an initial value determined by the manufacturer. The pressure is then increased gradually. Once water is observed on the external surface, the pressure is recorded and the test terminated. This is the water entry pressure.

\subsubsection{Expression of results}

The pressure shall be recorded in kilopascals (millimetres mercury).

8.2.4.6 Test report and additional information

The test report shall include the mean and standard deviations of the water entry pressure of the sample prostheses, and the details required by 4.9.1.

Additional information, including the rate of pressure increase, shall be recorded together with the details required by 4.9 .2 .

8.3 Determination of strength

NOTE Separate tests, applying a unidirectional stress, are required for determining the longitudinal and the circumferential tensile strengths of a sample prosthesis. Both tests shall be applied when appropriate.

8.3.1 Determination of circumferential tensile strength (A)

8.3.1.1 Principle

The sample prosthesis in its tubular form is placed onto two rounded pins. It is then stretched at a uniform rate until the yield and/or break point is reached. The test is a modification of ISO 5081.

\subsubsection{Apparatus}

Apparatus to be used include:

a) a tensile testing machine meeting the requirements of ISO 5081, having a constant rate of traverse, and with appropriately sized pins and suitable holders over which the sample prosthesis may be threaded: a suitable example is given in figure $4 \mathrm{a}$ ) and b);

b) a measuring device accurate to $\pm 0,5 \mathrm{~mm}$, e.g. a ruler or vernier calipers;

c) apparatus to measure the relaxed internal diameter (see 8.5).

8.3.1.3 Sampling

Sampling shall be in accordance with clause 7 .

24 


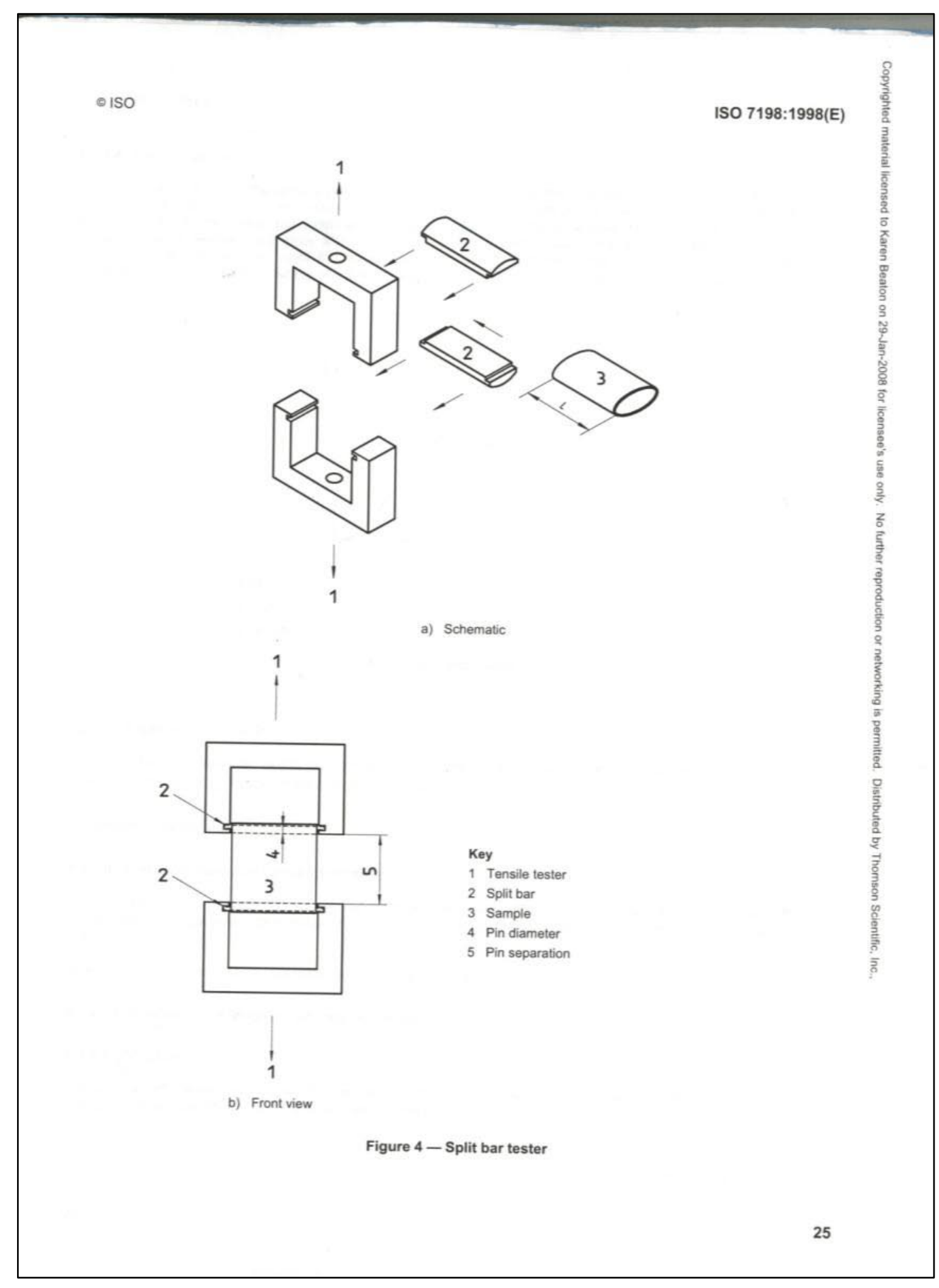




\subsubsection{Test procedure}

Cut a test specimen from the sample prosthesis with a length not less than the nominal relaxed internal diameter (8.5). After careful removal of any crimp, measure and record the length of the specimen $(L)$ in millimetres to an accuracy of $\pm 0,5 \mathrm{~mm}$. Thread the specimen over the two pins. Care should be taken to ensure that the specimen is not stretched or twisted, and slack should be kept to a minimum. Stretch the specimen at a steady rate of $50 \mathrm{~mm}$-min-1 to $200 \mathrm{~mm} \cdot \mathrm{min}^{-1}$ until the break point is reached. Determine the load at yield or break, i.e. the maximum load $\left(T_{\max }\right)$, to an accuracy of $\pm 2 \%$, and record the rate of extension (see figure 5 ), if appropriate.

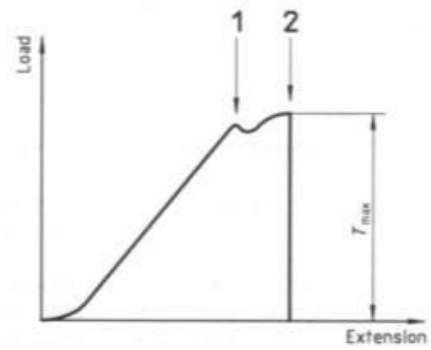

\subsubsection{Expression of results}

Caiculate the circumferential tensile strength of each sample, expressed as kilonewtons per millimetre, by dividing the maximum load $\left(T_{\max }\right)$ by the original length of the sample.

Maximum load/Length $=\frac{T_{\max }}{2 L}$

\subsubsection{Test report and additional information}

The test report shall include the mean and standard deviations of the circumferential strength of the sample prostheses, the strain rate with rationale if not within the specified range (see 8.3.1.4), and the details required by 4.9.1.

Additional information shall be recorded in accordance with 4.9.2.

8.3.2 Determination of longitudinal tensile strength (A)

8.3.2.1 Principle

The sample prosthesis in its tubular form is placed with its ends in suitable jaws. It is then stretched at a uniform rate until the yield and/or break point is reached. The test is a modification of ISO 5081. 


\section{Appendix C: Final Transverse Test Fixture Engineered}

\section{Drawings}

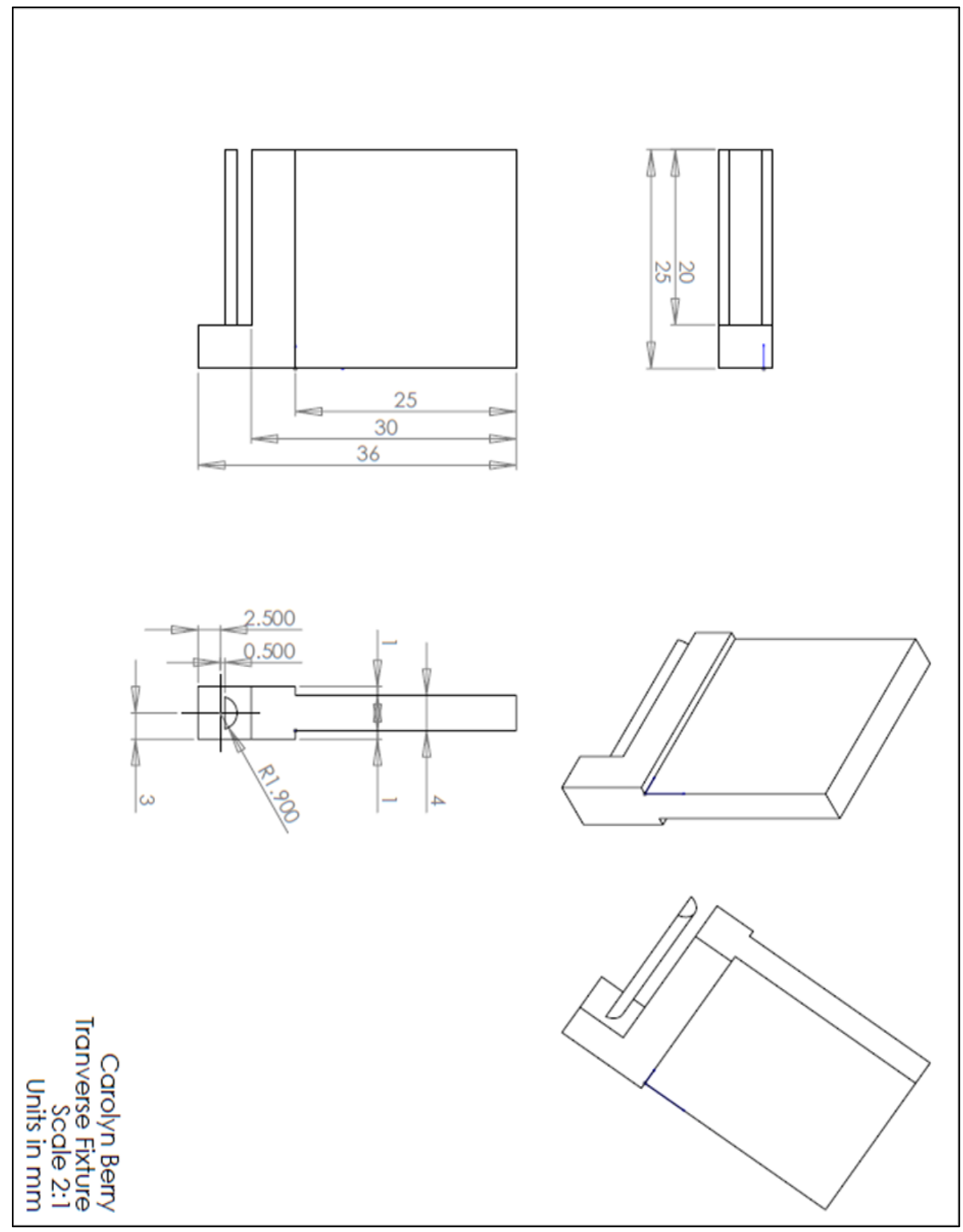




\section{Appendix D: Protocol for Testing Tubular Polymers using}

\section{Transverse Test Fixture on Instron In-Spec Machine}

1. Prepare sample of ePTFE or PLGA:

a. Cut a 5-10 mm piece of tubing.

b. Use drill press, located in the manufacturing lab in building 41, to cut $1 / 8$ " radius curves into each side of the sample:

i. Attach $1 / 4$ " hollow pin to drill press.

ii. Measure 1/8” down the length of the tubular sample and mark with a pencil.

iii. Place material under drill press and align the mark on the sample with the outer most edge of the hollow pin (see Figure 1).

iv. Pull down on drill press to create a $1 / 8$ " radius semi circular cut on the end of the tube.

v. Turn the tube to the other end and repeat steps ii through iv.

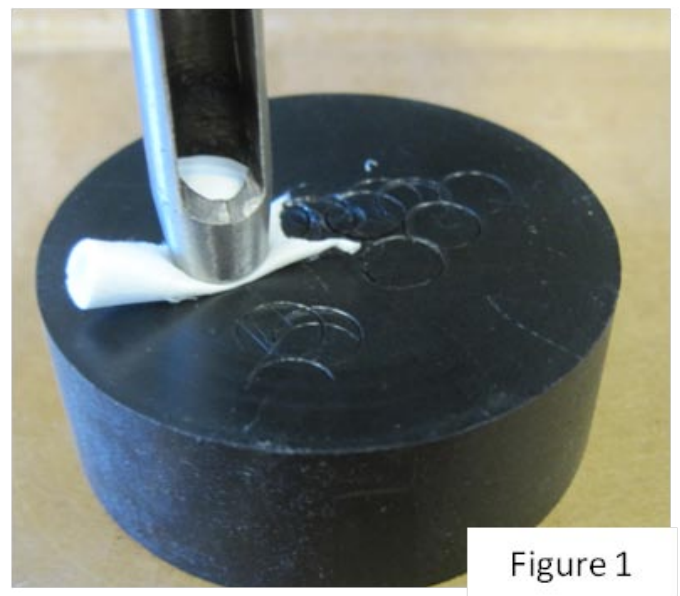

*Note: Please get permission from Martin Koch or Dave Laiho before using drill press.

c. Take sample to Building 192-330, where the Instron In-Spec 2200 is located. 
2. Turn on Instron machine using the switch located in the back, and turn on the Palm Pilot PDA by pressing the black button with the green dot.

3. Measure the width (w) of sample, which is the smallest distance between the two curves made by the drill press.

4. Measure the thickness $(t)$ of the wall of the sample.

5. On the PDA, touch Home (house icon), then touch "In-Spec".

6. Attach one connection grip of the test fixture to the Instron grips closest to the load cell.

7. Slide material sample onto the rod of the connection grip.

8. Insert the rod of the other connection grip into the material sample so the flat sides of each rod are flush against each other.

9. Move the Instron grips, using the arrowed switch button on top of the machine, close enough together to insert the loose connection grip of the test fixture into the empty grip of the Instron.

10. Secure the connection grip into the grip of the Instron.

11. Adjust the sample on the two rods so that the widest ends of the tube are

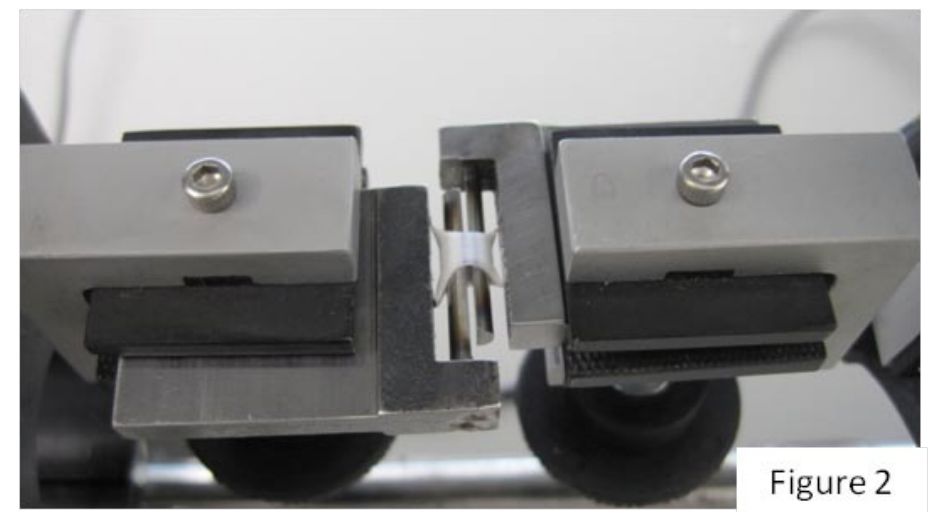
against the round parts of the rod.

12. Move the Instron grips far enough apart so the material sample is taught on the rods, but is not subjected to excessive force. This should look similar to Figure 2. 
13. Measure the gage length (1) of the sample by measuring with calipers the distance between the two rods, as shown in Figure 3.

14. On the PDA, zero the balance by selecting the "bal" icon on the top right corner of the screen.

15. Make sure the switch on

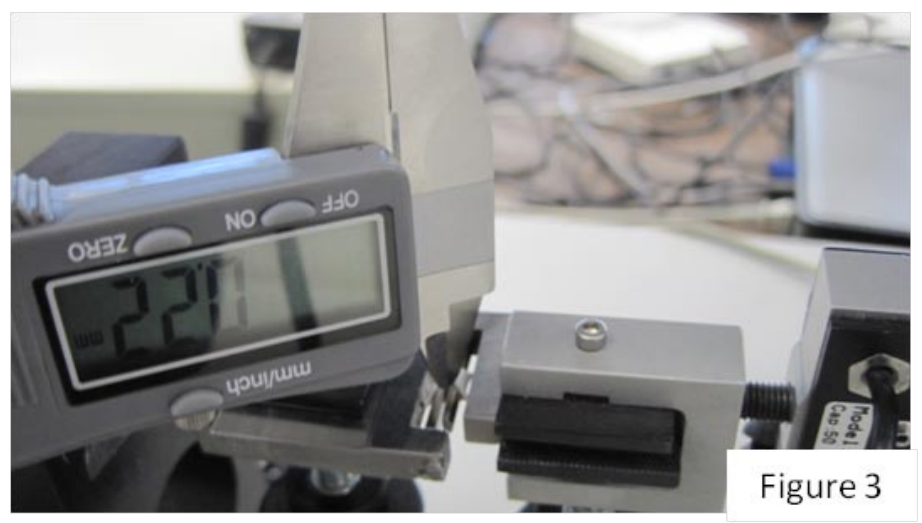
the side of the Instron machine is toward "toggle"

16. Also ensure that the correct direction is selected to pull the connection grips away from each other on the switch below "toggle".

17. Select the "start" icon on the PDA to begin testing.

18. Press the green button on top of the Instron machine.

19. Watch the graph on the PDA as well as the material sample to look for fracture.

20. Once fracture occurs, and the force over time curve on the graph begins to slope downward, press the green button again to stop the test.

21. Select the "stop" icon on the PDA then a window will appear to save the test. Name the test appropriately so it can easily be found later.

22. Repeat numbers 1-21 until desired number of samples have been tested.

23. To export data from PDA, select the "home" icon.

24. Select the "hot sync" icon on the menu.

25. Tap the screen of the PDA again to begin sync to the accompanying laptop. 
26. On the desktop of the laptop, open program called "Handheld" with an icon showing three square blocks.

27. Make sure "time, extension, load" is selected then open saved file name from PDA.

28. Save the file to the computer as a ".csv" so the file may be opened in Microsoft Excel.

29. Turn off the Instron machine and PDA.

30. Find the ultimate tensile strength and elastic modulus in Excel:

a. Open test file in Excel.

b. Columns A, B, and C should be labeled "Time”, "Extension”, and "Load", respectively.

c. Label column D “Strain” and column E "Stress”.

d. For column D, the extension in each row should be divided by the measured gage length of the sample to get the Strain.

e. For column E, the load in each row should be divided by the calculated cross-sectional area of the test sample.

i. To calculate the cross-sectional area, multiply the product of the sample width and thickness by two.

f. Plot a stress-strain curve using columns D and E.

g. The maximum stress is equal to the ultimate tensile strength (MPa).

h. To calculate the elastic modulus, plot a trendline on the initial upwards slope of the stress strain curve. The slope of the trendline is equal to the elastic modulus (MPa). 


\section{Appendix E: Protocol for Testing Tubular Polymers using}

\section{Instron In-Spec Machine (Current Method of Testing)}

1. Turn on Instron Tester (switch on the right side)

2. Secure safety locks

in the downward

position (red circle

on Figure 1) to avoid

grips running into

each other

3. Turn on PDA (black button with the green

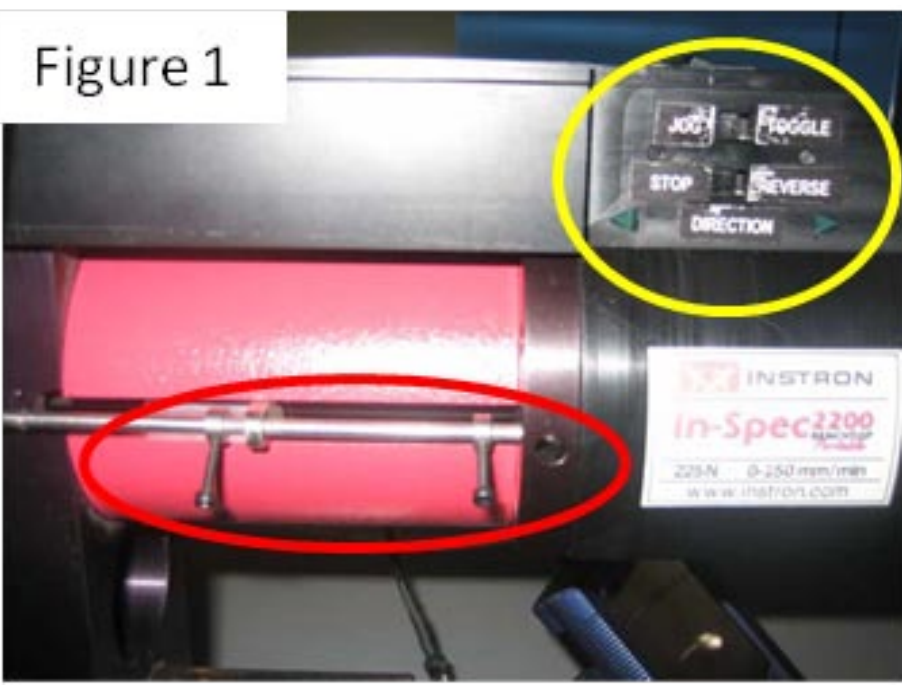
dot)

a. Touch Home (house looking icon)

b. Touch "In-Spec"

c. Adjust scale by touching the scale button on the bottom of the screen if necessary. The scale can be adjusted throughout the testing process as well.

4. Cut the sample longitudinally down the middle as shown in figure 2. (Use scissors)

5. Prepare the sample

FOR TESTING IN THE LONGITUDINAL DIRECTION:

a. Measure the thickness $(\mathrm{t})$ 
b. Measure the width (w): the length of the sample running perpendicular to the cut

c. Load sample into

\section{Figure 2}

Instron grips so that

the length of the

sample (the plane

along which the cut

was made) runs

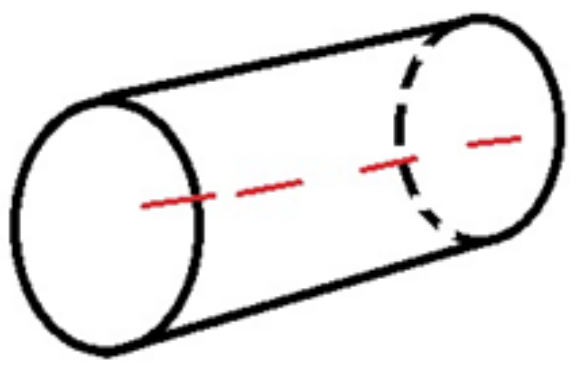

between the Instron grips

i. Place proximal end into the clamp that remains stationary

ii. Tigthen the clamp on a small part of the sample, but enough to hold it in place

iii. Repeat clamping process with the distal end of the moving clamp

1. Move the clamps so that the test sample is taut, but not stretched, by switch the machine to "jog” in the direction desired, then pressing the green button until the ideal position is reached

d. Measure the gage length (1): the distance between the Instron grips, or in other words, the length of the sample showing between the grips.

\section{FOR TESTING IN THE TRANSVERSE DIRECTION:}

e. Measure the thickness $(\mathrm{t})$

f. Measure the width (w): the distance along the line that was cut on tube 
g. Load sample into Instron grips so that each end of the sample that was created by cutting the tube is inserted into each clamp of the Instron grips

i. Place proximal end into the clamp that remains stationary

ii. Tigthen the clamp on a small part of the sample, but enough to hold it in place

iii. Repeat clamping process with the distal end of the moving clamp

1. Move the clamps so that the test sample is taut, but not stretched, by switch the machine to "jog” in the direction desired, then pressing the green button until the ideal position is reached

h. Measure the gage length (1): the distance between the Instron grips, or in other words, the length of the sample showing between the grips.

6. Zero the balance and load using the "bal" button (icons on top right of screen)

7. Ensure switch is pointing in the correct directions (since the sample is in tension, the arrow should be pointing to the right.) - "test direction” button

8. Ensure that "jog" (yellow circle on Figure 1) switch is selected

9. Hold down green button to apply a small load

10. Switch to "toggle"

11. Touch "start" on the PDA to start data collection

12. Press the Green button to start the tensile testing machine(green circle on Figure 3) 
13. Wait for data to plateau, then hit stop on the PDA

14. Push the green button to stop the test

15. Save the data as something that distinguishes your piece (there are lots of data

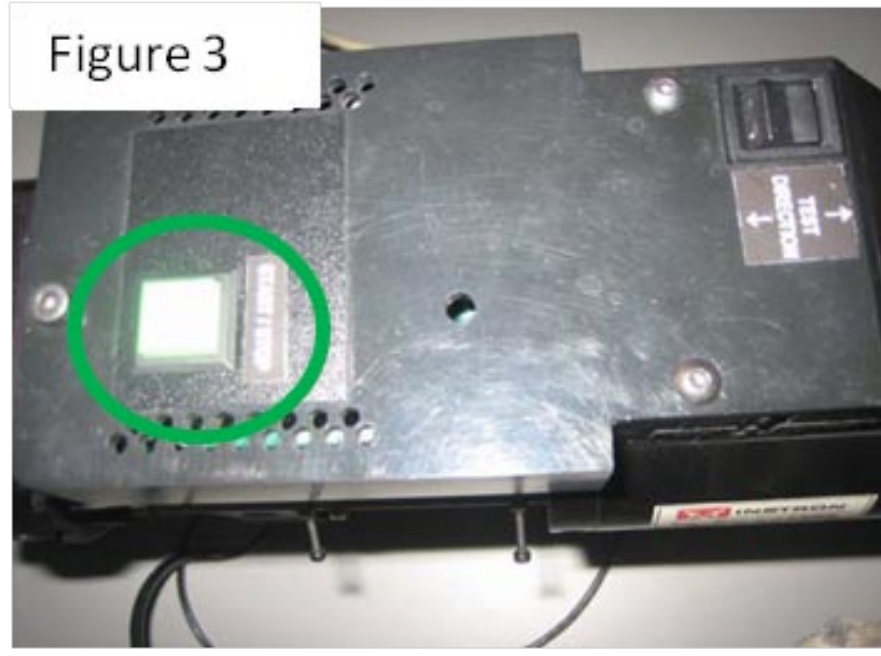
saved so make it unique)

16. Repeat for all test samples

17. Touch "Home" on the PDA

18. Touch "HotSync"

19. Touch icon that has two arrows in a circular image that will connect PDA to laptop

20. On the laptop, select desktop item handheld (three blocks)

21. Click “time, extension, load” on laptop to select data arrangement

22. Select “open” on laptop

23. Open folder on desktop "Shortcut to Backup"

24. Open your file

25. Save as filename.csv so it can be opened in excel

a. If performing tests at different times make sure to note at what speed the load is being applied (i.e. $1 \mathrm{~mm} / 10 \mathrm{sec}$ ). This can be checked by looking at your data. The extension per second should be the same for each sample. 
b. When performing each test, NEVER touch the home button until after the file has been saved on the PDA or the data will be lost...forever, and you will have to trash that sample

26. From excel, arrange data in order to plot a stress-strain curve

27. Using the graph, calculate the Elastic modulus and UTS of each sample

a. Open test file in Excel.

b. Columns A, B, and C should be labeled “Time”, “Extension”, and "Load”, respectively.

c. Label column D “Strain” and column E “Stress”.

d. For column D, the extension in each row should be divided by the measured gage length of the sample to get the Strain.

e. For column E, the load in each row should be divided by the calculated cross-sectional area of the test sample.

i. To calculate the cross-sectional area, multiply the width of the sample by the thickness of the sample.

f. Plot a stress-strain curve using columns D and E.

g. The maximum stress is equal to the ultimate tensile strength (MPa).

h. To calculate the elastic modulus, plot a trendline on the initial upwards slope of the stress strain curve. The slope of the trendline is equal to the elastic modulus (MPa). 


\section{Appendix F: Results of ePTFE and PLGA Experimental}

\section{Studies Comparing Transverse Fixture vs. Current Method of}

\section{Testing}

\begin{tabular}{|r|r|r|r|}
\hline \multicolumn{5}{|c|}{ ePTFE (Transverse Fixture) Data } \\
\hline Sample & Xsection $\left(\mathrm{mm}^{\wedge} 2\right)$ & UTS (MPa) & E (MPa) \\
\hline 1 & 4.233 & 7.708 & 6.488 \\
\hline 2 & 3.424 & 9.866 & 17.234 \\
\hline 3 & 3.806 & 7.309 & 13.023 \\
\hline 4 & 1.656 & 8.681 & 11.587 \\
\hline 5 & 1.095 & 9.227 & 4.607 \\
\hline 6 & 3.526 & 12.454 & 12.344 \\
\hline 7 & 2.934 & 10.104 & 1.938 \\
\hline Average & 2.953 & 9.336 & 9.603 \\
\hline Std Dev. & 1.158 & 1.722 & 5.397 \\
\hline
\end{tabular}

\begin{tabular}{|r|r|r|r|}
\hline \multicolumn{5}{|c|}{ ePTFE (Current Method) Data } \\
\hline Sample & Xsection $\left(\mathrm{mm}^{\wedge} 2\right)$ & UTS (MPa) & E (MPa) \\
\hline 1 & 3.432 & 4.213 & 4.981 \\
\hline 2 & 3.709 & 5.453 & 7.321 \\
\hline 3 & 3.514 & 4.384 & 5.991 \\
\hline 4 & 3.218 & 6.080 & 8.116 \\
\hline 5 & 3.125 & 5.977 & 6.584 \\
\hline 6 & 2.121 & 7.757 & 10.464 \\
\hline Average & 3.186 & 5.644 & 7.243 \\
\hline Std Dev. & 0.562 & 1.299 & 1.911 \\
\hline
\end{tabular}




\begin{tabular}{||r|r|r|r|}
\hline \multicolumn{5}{||c|}{ PLGA (Transverse Fixture) Data } \\
\hline Sample & Xsection (mm^2) & UTS (MPa) & E (MPa) \\
\hline 1 & 2.448 & 3.501 & 13.313 \\
\hline 2 & 2.184 & 4.611 & 10.467 \\
\hline 3 & 3.410 & 3.966 & 8.337 \\
\hline 4 & 3.704 & 4.076 & 8.811 \\
\hline 5 & 3.657 & 3.999 & 4.831 \\
\hline 6 & 4.439 & 3.163 & 10.986 \\
\hline 7 & 2.870 & 4.587 & 8.811 \\
\hline 8 & 4.469 & 3.856 & 5.703 \\
\hline 9 & 2.890 & 3.978 & 4.776 \\
\hline 10 & 3.754 & 3.410 & 11.338 \\
\hline 11 & 3.078 & 3.935 & 8.565 \\
\hline 12 & 2.488 & 3.861 & 9.040 \\
\hline 13 & 3.805 & 3.500 & 11.841 \\
\hline 14 & 2.727 & 3.231 & 10.499 \\
\hline Average & 3.280 & 3.834 & 9.094 \\
\hline Std Dev. & 0.722 & 0.440 & 2.589 \\
\hline
\end{tabular}

\begin{tabular}{|r|r|r|r|}
\hline \multicolumn{5}{||c|}{ PLGA (Current Method) Data } \\
\hline Sample & Xsection (mm^2) & UTS (MPa) & E (MPa) \\
\hline 1 & 2.042 & 2.925 & 62.323 \\
\hline 2 & 2.935 & 3.577 & 28.334 \\
\hline 3 & 4.261 & 3.004 & 24.372 \\
\hline 4 & 4.335 & 2.770 & 35.881 \\
\hline 5 & 3.768 & 3.387 & 25.793 \\
\hline 6 & 3.416 & 4.449 & 85.873 \\
\hline 7 & 4.830 & 2.983 & 29.983 \\
\hline 8 & 3.710 & 3.244 & 39.022 \\
\hline 9 & 3.585 & 3.346 & 19.847 \\
\hline 10 & 4.351 & 2.844 & 39.089 \\
\hline 11 & 3.653 & 2.977 & 41.867 \\
\hline 12 & 4.483 & 2.610 & 42.748 \\
\hline 13 & 4.913 & 2.728 & 27.657 \\
\hline 14 & 2.428 & 2.333 & 36.804 \\
\hline 15 & 2.326 & 2.947 & 28.351 \\
\hline 16 & 3.420 & 2.715 & 17.441 \\
\hline 17 & 1.721 & 3.115 & 83.194 \\
\hline Average & 3.540 & 3.056 & 39.328 \\
\hline Std Dev. & 0.968 & 0.472 & 19.979 \\
\hline
\end{tabular}




\section{Appendix G: Final Longitudinal Test Fixture Engineered}

\section{Drawings}

Mandrel

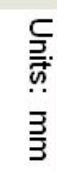
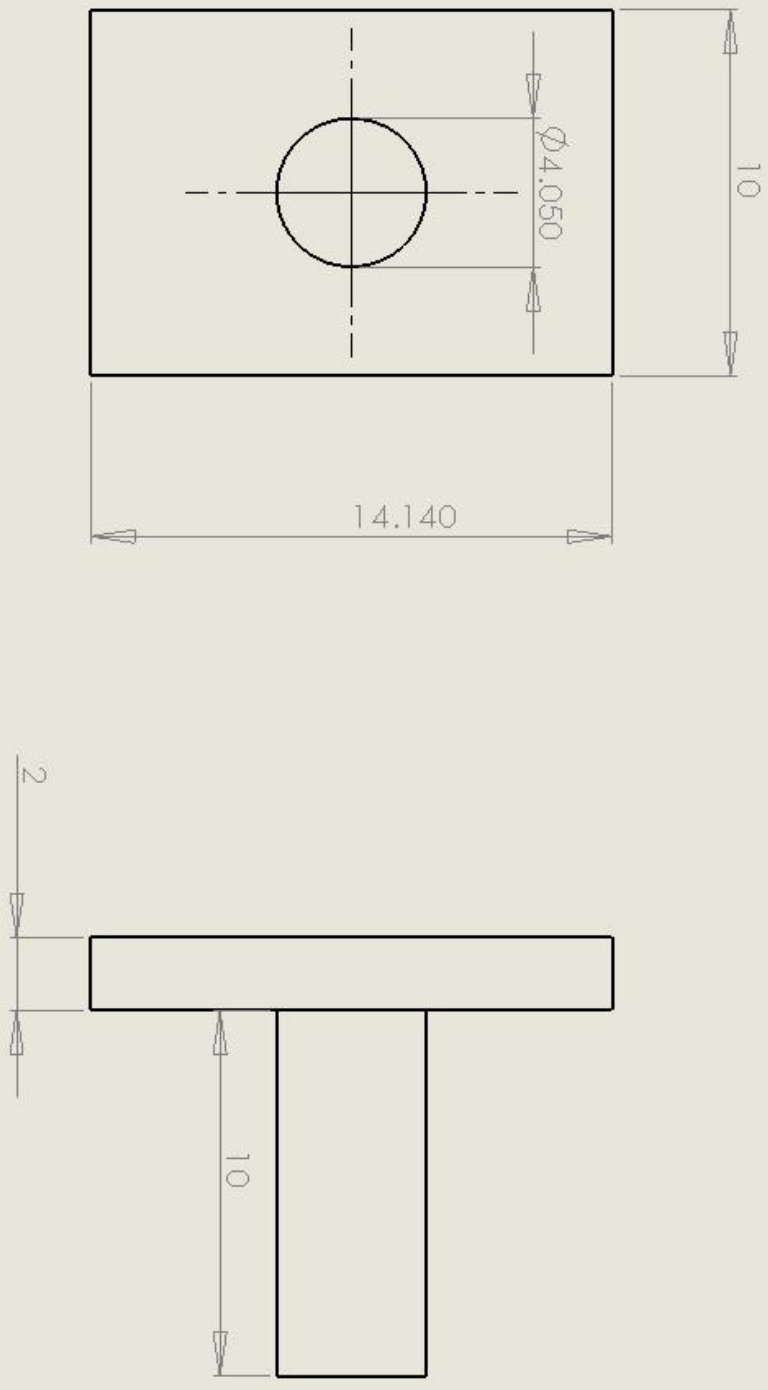


\section{Top/Bottom Part of Fixture}
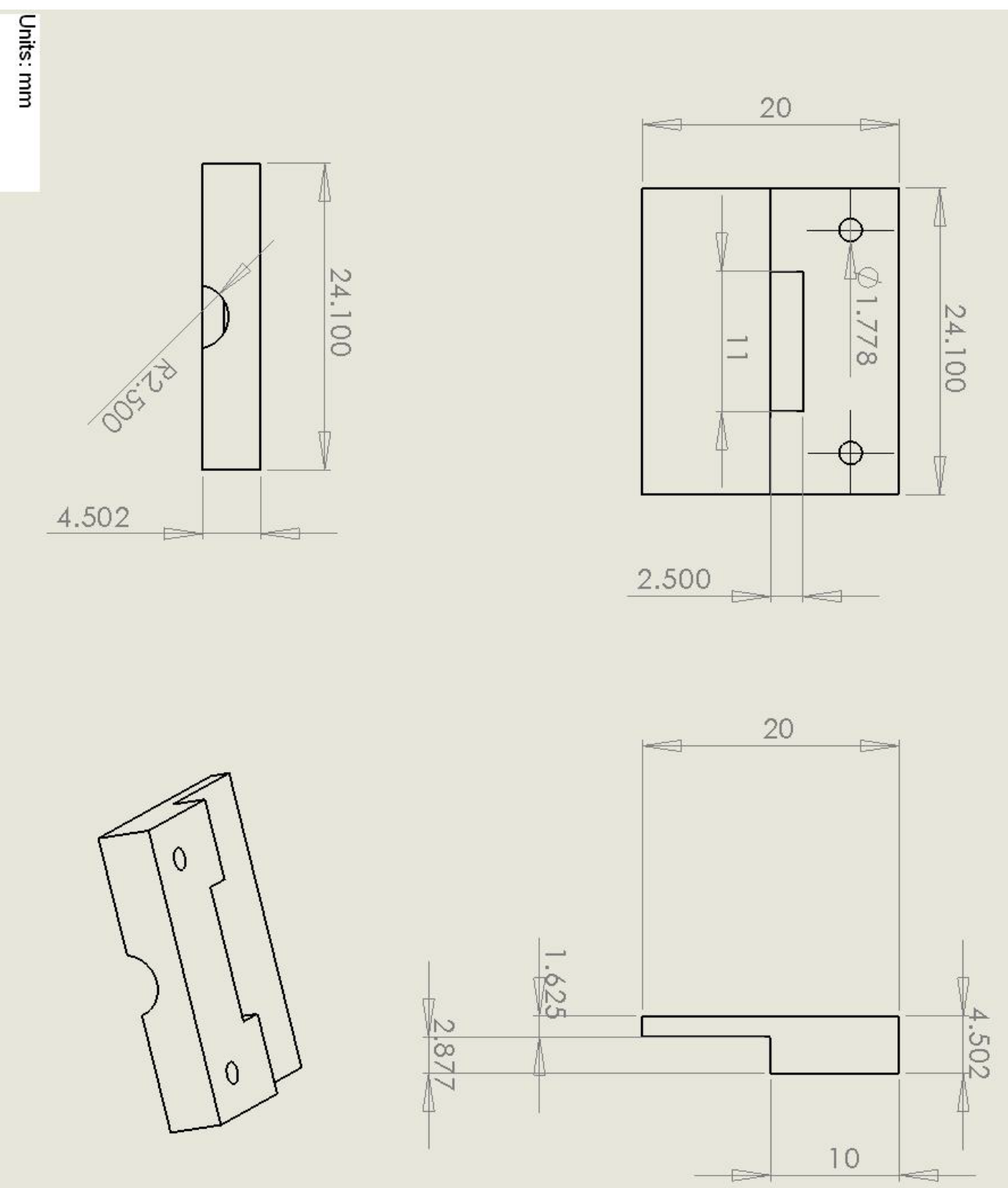